\title{
Traditional use and management of NTFPs in Kangchenjunga Landscape: implications for conservation and livelihoods
}

\author{
Yadav Uprety ${ }^{1 *}$, Ram C. Poudel ${ }^{2}$, Janita Gurung ${ }^{3}$, Nakul Chettri ${ }^{3}$ and Ram P. Chaudhary ${ }^{1,4}$
}

\begin{abstract}
Non-timber Forest Products (NTFPs), an important provisioning ecosystem services, are recognized for their contribution in rural livelihoods and forest conservation. Effective management through sustainable harvesting and market driven commercialization are two contrasting aspects that are bringing challenges in development of NTFPs sector. Identifying potential species having market value, conducting value chain analyses, and sustainable management of NTFPs need analysis of their use patterns by communities and trends at a regional scale. We analyzed use patterns, trends, and challenges in traditional use and management of NTFPs in the southern slope of Kangchenjunga Landscape, Eastern Himalaya and discussed potential implications for conservation and livelihoods. A total of 739 species of NTFPs used by the local people of Kangchenjunga Landscape were reported in the reviewed literature. Of these, the highest number of NTFPs was documented from India (377 species), followed by Nepal (363) and Bhutan (245). Though the reported species were used for 24 different purposes, medicinal and edible plants were the most frequently used NTFP categories in the landscape. Medicinal plants were used in 27 major ailment categories, with the highest number of species being used for gastro-intestinal disorders. Though the Kangchenjunga Landscape harbors many potential NTFPs, trade of NTFPs was found to be nominal indicating lack of commercialization due to limited market information. We found that the unsustainable harvesting and lack of marketing were the major constraints for sustainable management of NTFPs sector in the landscape despite of promising policy provisions. We suggest sustainable harvesting practices, value addition at local level, and marketing for promotion of NTFPs in the Kangchenjunga Landscape for income generation and livelihood improvement that subsequently contributes to conservation.
\end{abstract}

Keywords: Traditional knowledge, Medicinal plants, Trade, Potential species, NTFP policy, Sustainable use and management

\section{Background}

Non-timber forest products (NTFPs) are the most important provisioning services people obtain from forest ecosystems [1]. The importance of NTFPs in rural livelihoods and forest conservation has been well recognized as they provide income generation opportunities to millions of people around the world [2-5], and they are also a major source of supplementary food, medicines,

\footnotetext{
*Correspondence: yadavuprety@gmail.com

'Research Centre for Applied Science and Technology, Tribhuvan University, Kirtipur, Kathmandu, Nepal

Full list of author information is available at the end of the article
}

fibre, and construction materials [6, 7]. In developing countries, biological resources obtained from forests, mostly NTFPs, may contribute as much as $20-25 \%$ of income to rural people [7]. However, the economic potential of NTFPs is highly contextual and depends on a combined set of socio-cultural, ecological, geo-political, and economic conditions. Nevertheless, access to market/ commercialization of NTFPs and sustainable harvesting are two important aspects that need attention for sustainable development of the NTFP sector (also see [8]).

The ecological diversity of the Himalaya makes the area a habitat of a vast range of NTFPs. In the Himalayan 
region, harvesting NTFPs is a tradition that also contributes significantly to the local economy. Some NTFPs play an important role in traditional health care systems, while others have important cultural values and are sources of food and housing material [9-11]. Among all categories of NTFPs, medicinal plants have received much focus while the contribution of other categories of NTFPs has been overlooked. For example, the contribution of wild edible plants towards food security and income generation has been undervalued in Nepal [12].

Common threats to NTFPs in the Himalayan region include unsustainable harvesting and habitat loss due to land use change, deforestation and over-grazing [13, 14]. Several other challenges have also been identified for sustainable management of NTFPs, such as policies that are ambiguous or poorly implemented due to the lack of resources, lack of comprehensive information on the ecology of the species and its socio-ecological impacts, and poor infrastructure for bioprospecting [15-17]. However, unsustainable harvesting is one of the major issues that affects ecological processes at many levels, from individual and population to community and ecosystem [2, 18]. Commercialization of NTFPs is another important aspect involving different processes such as production, collection, processing, storage, transport, marketing, and sale. Marshall et al. [19] found that product marketing and sale were the most important of all factors that constrained overall success of NTFPs commercialization. However, Ghate et al. [20] found a clear relationship between the degree of proximity to the market and NTFP dependence; remote places with low market access had high NTFPs dependency.

The demand for NTFPs is increasing not only in local markets, but also in international markets. Therefore, some important steps to facilitate integration of NTFPs into the development agenda that benefits local communities include identifying potential species having trade value and conducting research on their ecology and sustainable harvest levels; conducting analyses on value chain and use patterns; and analyzing trends and challenges in marketing and management [21]. Here we focus on these aspects of NTFPs in the Kangchenjunga Landscape within the Eastern Himalaya [22] and explore the implications for conservation and livelihoods.

\section{Methods}

\section{Study area}

The Kangchenjunga Landscape is a transboundary landscape shared by Bhutan, India, and Nepal. It is one of the richest landscapes in the Hindu Kush Himalaya $(\mathrm{HKH})$ in terms of cultural and biological diversity and forms part of the Himalaya Biodiversity Hotspot, one of 34 global Biodiversity Hotspots [23]. It extends over 25,000 sq. km within $26^{0} 21^{\prime} 40.49^{\prime \prime}$ to $28^{0} 7^{\prime} 51.25^{\prime \prime}$ North latitudes and $87^{\circ} 30^{\prime} 30.67^{\prime \prime}$ to $90^{\circ} 24^{\prime} 31.18^{\prime \prime}$ East longitudes (Fig. 1). The altitude in the landscape ranges from 50 masl in the south to 8,586 masl, the height of Mount Kangchenjunga-the world's third highest peak. Vegetation zones in the landscape is comprised of tropical, subtropical, temperate, subalpine, alpine, and nival.

The Kangchenjunga Landscape provides a range of ecosystem services that supports millions of people [24]. However, like many other landscapes worldwide, biodiversity and ecosystems within the landscape face threats mainly from anthropogenic pressures [25] and global climate change [26]. As a result, the people living in the landscape are economically, physically, and socially vulnerable $[25,27]$.

Recognizing the global and regional significances and challenges that lie within this landscape, the Kangchenjunga Landscape Conservation and Development Initiative (KLCDI) has been initiated by the governments of Bhutan, India and Nepal to achieve biodiversity conservation and sustainable development by applying ecosystem approaches to transboundary landscape management [22]. One of these priority areas is sustainable utilization of NTFPs in the Kangchenjunga Landscape. Several species of high value NTFPs that are also threatened are found in the landscape such as Chiraito (Swertia chirayita), Panch aunle (Dactylorhiza hatagirea), Kutki (Neopicrorhiza scrophulariiflora), Laghupatra (Podophyllum hexandrum) and Lauth salla (Taxus wallichiana).

\section{Data collection and analysis}

We reviewed scientific studies published in journals and books on traditional uses of NTFPs in the Kangchenjunga Landscape. Various online databases were used (ISI Web of Science, Scopus, and Google Scholar) using specific search terms such as 'non-timber forest products', 'medicinal plants', 'wild edible plants', and 'Kangchenjunga Landscape', 'Nepal,' 'India,' 'Sikkim,' 'Darjeeling', and 'Bhutan'. We also explored hard copies of relevant publications. We reviewed a total of 47 publications and one database to enumerate the NTFPs in the Kangchenjunga Landscape. The precision of species identification in this review was dependent on the original source. However, we verified currently accepted name(s) in online nomenclature sources (http://www. theplantlist.org and http://www.tropicos.org). Vernacular names when available have also been provided. A master list was produced providing Linnaean taxonomy, vernacular name(s), mode(s) of use, and reference(s) for each species (Table 1). We also collected trade data and reviewed policy documents on NTFPs of Bhutan, India and Nepal.

We listed ailments as mentioned in the publications but we followed the method proposed by Cook [28] to classify plants according to the different ailment categories they used to cure. However, in some cases Cook's categories were not precise enough and plants were assigned to 


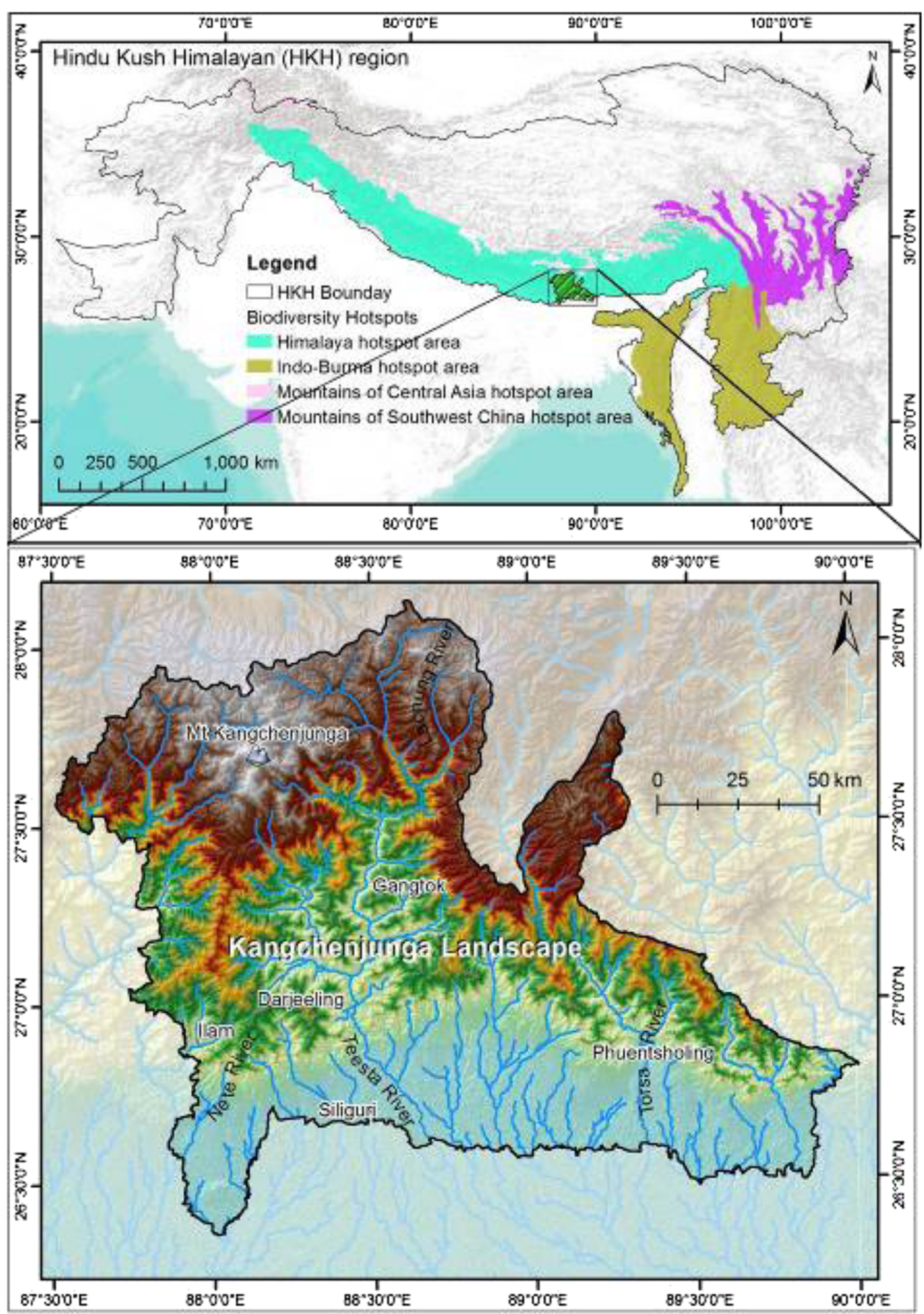

Fig. 1 The Kangchenjunga Landscape in the Eastern Himalaya 
Table 1 NTFPs used by the local people of the Kangchenjunga Landscape, Eastern Himalaya
SN Botanical name $\quad$ Habit $\quad$ Family

Distribution

Part(s) used, Use(s), Location and Reference(s)*

\section{Angiosperms}

1 Justicia adhatoda L.

Shrub

Acanthaceae

Asuro (Np); Bashakha (Dz):

Jantrashi (Me); Khateermu

(Sh); Vasakdog (S)

Acanthaceae

Strobilanthes cusia (Nees) Herb

Kuntze

3 Viburnum erubescens Wall. Shrub

Trianthema portulacastrum Herb L.

5 Alangium salvifolium (L.f.) Tree Wangerin

6 Achyranthes aspera L. Herb

Achyranthes bidentata Herb Blume

8 Alternanthera sessilis (L.) DC. Herb

9 Amaranthus spinosus L., Herb

10 Amaranthus spp.

11 Amaranthus tricolor $\mathrm{L}$.

Herb

2 Amaranthus viridis L.

Herb

3 Celosia argentea L.

4 Allium sp.

15 Allium wallichii Kunth

Herb
Adoxaceae

Aizoaceae

Alanginaceae

Amaranthaceae

Amaranthaceae

Amaranthaceae

Amaranthaceae

Amaranthaceae

Amaranthaceae

Amaranthaceae

Amaranthaceae

Amaryllidaceae

Amaryllidaceae
Asaray (Np)

Seto Punarnava (Np)

Dhela (S): Asare (Np)

Apamarga, Ulte kuro (Np)

Ankhlay Jhar, Datiwan (Np)

Saranchi Saag (Np); Garundi (S)

Janum arak (S); Lude (Np)

Zimtsi (Dz); Naam (Sh)

Gandhari (S); Lude (Np)

Bhale Phul, Sahastrajadi (Li)

Lagok (Dz/Sh)

Wild Garlic (Eg); Palengu (Gr); Gokpa, Koje, Ri Gokpa (Km, Sh); Ban Lasun, Ban Pyaj, Jimbu, Jimbujhar (Np); Bathatuva (Ri)
$500-1600$

Roots: Extract taken to cure cholera and epilepsy (KL Nepal: [53]). Roots, bark and leaves: Used as insecticide, expectorant, and antispasmodic. Used as remedy for asthma, cough, fever, gonorrhea leprosy, and phthisis (Sikkim: Database). . Tender shoots: Used to treat asthma (Jhapa: [66]; Panchthar: [67]). Leaves: Extract given orally to cure wheezing in children (Jhapa: [68]). Decoction used in bronchitis, cold, and veneral diseases (Jhapa: [66]). Paste applied on abdomen and vagina just minutes before childbirth for easy delivery (Sikkim: [69]). Leaves and shoots: Taken orally against fever, headache and bodyache (Darjeeling: [52]). Flowers: Edible (KL Bhutan: [70]) and also and bodyache (Darjeeling: [52]). Flowe
used as medicine (KL Bhutan: [71]).

Leaves: Use to extract dye (KL Bhutan: [70]).

2000-3500 Seeds: Edible (Darjeeling: [72]).

150-300

Young shoots: Used as vegetable (Jhapa: [92]).

$150-350$

$800-2300$

Bark: Paste used for abortion and antifertility (Jhapa: [66]). Fruit: Edible (Jhapa: [92])

Whole plant: Juice taken in cough (Panchthar: [67]; llam: [73]), dropsy, piles, stomachache (llam: [73]) and diarrhoea (Panchthar: [67]).

200-2100 Roots and stem: Juice used as diuretic and also given in rheumatism (Darjeeling: [74]; Sikkim: [75]; Ilam: [73, 76, 77]) and hypertention (llam: [73, 76, 77]).

200-2000

Roots: Pounded with seeds of Foeniculum vulgare and taken orally to cure piles (Jhapa: [66]). Leaves and twigs: Decoction taken to cure fever (Darjeeling: [78]).

150-1200 Roots: Extract used as diuretic (KL Nepal: [53]). Decoction taken with warm water to check excessive bleeding at post delivery stage (Jhapa: [66]). Leaves: Used as vegetable (Jhapa: [92]). Eaten as curry to treat burns, boils and as Used as vegetable (Jhapa:

Whole plant: Used as spices (KL Bhutan: [70]).

Leaves: Used to stop diarrhea (Sikkim: [11]). Seeds: Taken to cure gastric problems, fried beaten seeds with butter are given to pregnant women to lessen pregnancy pains (Sikkim: [11]).

$150-1200$

Young shoots and leaves: Used as vegetable (Jhapa: [92]). Juice with sugar taken to cure colic and as laxative (Darjeeling: [74]).

$500-1600$

Les: Juice administered orally in diarrhoea and dysentery (Sikkim: [79]).

Whole plant: Used as medicine (KL Bhutan: [71]).

$2300-4800$

Plant: Used in digestion (Sikkim: [69]). Bulbs and aerial parts: Used as spice (Sikkim: [10, 80], Database; Taplejung: [81, 82]; Sikkim: Database). Bulbs: Boiled, fried in clarified butter and taken in cholera and diarrhea; chewed to treat cough, colds, and altitude sickness (Taplejung: [83]). Bulbs and flowers: Use to treat gastric disorders (Sikkim: [75]). 
Table 1 NTFPs used by the local people of the Kangchenjunga Landscape, Eastern Himalaya (Continued)

Tree

(Roxb.) B. L. Burtt \& A. W.

Hill

17 Lannea coromandelica (Houtt.) Merr.

18 Mangifera indica L.

Tree

Anacardiaceae

Anacardiacea

19 Mangifera sylvatica Roxb. Tree

20 Rhus chinensis Mill.

21 Rhus javanica Miller

Tree

Anacardiaceae

Anacardiaceae

23 Spondias pinnata (L. f.) Kurz Tree

24 Toxicodendron hookeri (K.C. Tree Sahni \& Bahadur) C.Y. Wu \& T.L. Ming

25 Annona squamata $\mathrm{L}$.

Apiaceae

27 Angelica sinensis (Oliv.) Diels Herb

28 Carum carvi L.

Anacardiaceae

Anacardiaceae

Annonaceae
Nepali Hug Plum (Eg); Phindruwa (Li);

Lapsi (Np); Amali (Nw); Pindumsi,

Pintupsi (Ri); Nying Sho Sha (Tb)

Doka (S)

100-1400

Thaijau (Me); UI (Np)

$300-700$

Amaro (Np)

Tarsishing (Sh)

Saripha (Np); Mandargom (S)

$500-900$ (Np); Sushavi (Sn); Chhonyo, Khoda Sh,Wi
Caraway (Eg); Chir (Gr-Mn); Ban Jeera

Aplacea $2500-5100$
Fruits: Edible (KL Bhutan: [70]). Edible and also used to make pickle (Darjeeling: [80], Sikkim: [10, 47, 72, 95]; llam: [94]; Taplejung: [88]). Used to treat cough, cold, tonsillitis, diabetes, worm infestation, and heart ailment [87].

Bark: Paste applied around bone fracture to set bone (Jhapa: [66]).

Plant: Effective for jaundice patient (Darjeeling: [105]). Fruits: Edible (KL Bhutan: [70]). Green unripe fruit skin crushed and drunk to get relief from stomachache (Jhapa: [68]). Bark: Given with bark of Anthocephalus chinensis, Madhuca longifolia and Gmelina arborea in diarrhea and dysentery (Jhapa: [66]). Leaves: Young leaves chewed during cough and sore throat (Sikkim: [79]).

Fruits: Used as laxative (Sikkim: [85]).Used to make sour pickle (Ilam: [94]).

Fruits: Edible (KL Bhutan: [70]). Used as medicine (KL Bhutan: [71]). Flowers and fruits: Use to extract dye (KL Bhutan: [70]).

Fruits: Edible and also used in the treatment of diarrhea, dysentery (Taplejung: [88]; Panchthar: [67]; llam: [73]) and stomach problems (Taplejung: [88]; llam: [73]). Dried and extract used in diarrhea, swellings and wounds (Sikkim: Database). Juice used as food preservative; juice boiled with water and mixed with raw egg and the mixture given to treat diarrhea (Sikkim: [1 1]) and dysentery (Darjeeling: [80]; Sikkim: [11]). Juice administered orally during blood dysentery (Sikkim: [79]). Boiled and isolated vinegar us [94]). Roots: Used to treat skin infection (Sikkim: [11, 75]). Bark: Decoction given to animals to remove worms (Sikkim: [11]). Latex: Used as antitetanus (Jhapa: Seeds: Used as medicine (KL Bhutan: [71]). Seed oil taken in cough and colds (Jhapa: [66]).

Flowers: Used to make curry and for flavoring (llam: [94])

Fruits: Edible (KL Bhutan: [70])

Bark: Juice drunk to control fever (Jhapa: [68]). Leaves and seeds: Used to kill lice (Panchthar: [67]). Thalamus is used as antifertility (Jhapa: [66]). Seeds: Useful in abortion and menstrual disorders (Jhapa: [66]). Roots and seeds: Paste applied on forehead during headache (Sikkim: [79]).

Roots: Used as medicinal (KL Bhutan: [71]).

Roots: Used as medicinal (KL Bhutan: [71]).

Fruits: Used as medicine (KL Bhutan: [71]). Leaves: Used as vegetable (Taplejung: [87]). 
Table 1 NTFPs used by the local people of the Kangchenjunga Landscape, Eastern Himalaya (Continued)

Ghortaapre, Gol patta, Gora taprey, Goltaprey (Np); Dalka chatumana (S)

$500-2100$

Dhaniya (Np)

$1000-2500$

31 Cortia depressa (D.Don) C. Herb Norman

Apiaceae

32 Heracleum nepalense D. Herb Don

Apiaceae

33 Heracleum wallichii DC

Apiaceae

Apiaceae

Heracleum candicans Wall. Herb ex DC

35 Heracleum dissectifolium K.T. Herb $\mathrm{Fu}$

36 Hydrocotyle himalaica P. K. Herb Mukh.

37 Hydrocotyle javanica Thunb. Herb

38 Hydrocotyle sibthorpioides Herb Lam.

39 Selinum wallichianum (DC.) Herb Raizada \& Saxena

40 Alstonia scholaris (L) R. Br. Tree

\section{Apiaceae}

Apiaceae

Apiaceae

Apiaceae

Apiaceae

Apocynaceae
Nhopchema (Sh): Tangkyun (Sh, Tb) Ghundu (WI) Chimphing (Np); Bhote-Jeera, Nafo (NpDl); Chimping (Np-Tb); Phaki, Thar (Sh); Chimbing, Chimping (Sh, Wl); Chapho Karpo, Zira Goepa (Tb)

Chimphing (Np)

$3600-4100$

Golpata (Np)

$1500-2500$

Golpatta (Np)

Tike Ghor Tapre (Np)

$600-2500$

Sunaga (Km); Bhutkesh (Np); Bhatauri, Dhaneli, Kalo Chhetaro (Np-DI); Tunak

$2700-4800$
Plant: Used to cure heating and tenderness of limb skin (Jhapa: [68]). Juice taken orally to get rid of pneumonia, fever (Sikkim: [75]), asthma (Uhapa: [68] Ilam: [76, 77]. Sikkim: Database, [69]), mental tension, urinary problems, and stomachache (llam: [76, 77]. Jhapa: [68]. Sikkim: [75]). Infusion massaged on the stomachache (llam. [76, 77], Thapa. [68], Sikkim. [75]). Infusion massaged on the forehead to relieve from headache (Jhapa. [66]). Leaves. Used for skin disease and improving memory (Sikkm. Database). Leaves and young shoots taken to cure tonsil (Darjeeling. [52]). Aerial parts mixed with young shoots of Justicia adhatoda and taken against diarrhoea (Darjeeling: [86]).

Seeds: Used as spice (llam: [76]) and in flatulence (Darjeeling: [91]). Leaves: Used in flatulence (Darjeeling: [91]). Green leaves used to enhance aroma on food (llam: [76]). Plant: Used in cough, bronchitis, reheumatism and urinary problem (llam: [76])

Leaves: Tender leaves used as vegetable (Taplejung: [88]). Seeds: Used as spice (Taplejung: [81]).

Sunaga, Habluwa (Km); Samben (Lp);

Plant: Used as good winter fodder (Taplejung: [81, 87]). Roots: Given to cattle as tonic; juice and roasted seeds taken in cough and diarrhoea (Panchthar: [67]; Ilam: [73, 77]). Flowers and fruit: Suggested to cure influenza and body ache (Sikkim: [75]). Fruit: Used as pickle and also in typhoid, nausea, and vomiting (Darjeeling: [78]). Seeds: Used in case of bodyache, headache, and faint (Panchthar: [67]; llam: [73]).

Roots: Used as medicine (KL Bhutan: [71]).Used as tonic and aphrodisiac (Darjeeling: [78]). Juice taken to cure diarrhea (Sikkim: [1 1]). Seeds: Taken in diarrhea and gastric disorders (Sikkim: [75]), used as chatni (Sikkim: [11]). Decoction of dried seeds taken in influenza (Darjeeling: [78]; Sikkim: [11]).

Whole plant: Used as medicinal (KL Bhutan: [71]).

Roots: Used as medicine (KL Bhutan: [71]).

Plant: Extract taken to cure pneumonia and throat infection (Sikkim: [75]). (Tb); Chyadukpa (Sh, WI)

Plant: Taken to cure throat problem (Sikkim: [75])

Plant: Useful in treatment of migrant and to improve memory (Panchthar: [67]).

Roots and fruits: Used for cuts, wounds, colic, gastritis, and intestinal pain (KL Nepal: [83]). Roots: Smoke inhaled in case of faint fever, and headche. Also used as fodder (Taplejung: [811). Decoction taken to get relief from pain and used as fodder (Taplejung: [81]). Decoction taken to get rellef from pain and fever (llam: [76]). Flowers and leaves: Smooked to treat headache (KL Nepa

Bark: Used in malarian fever and also given to lactating mothers for increasing milk (Jhapa: [66]). Extract used to treat piles (KL Nepal: [53]). Filtered bark juice used to cure leanness in man (Jhapa: [68]). Powder mixed with common salt and given to cattle to cure fever (Sikkim: [97]). Bark, latex and flowers: Used as tonic and in fever and leucoderma (Sikkim: [97]). 
Table 1 NTFPs used by the local people of the Kangchenjunga Landscape, Eastern Himalaya (Continued)

haranthus roseus

Shrub

Apocynaceae

Barhamase Phool, Sadabahar (Np):

Gofatbhiwar (Me)

42 Holarrhena pubescens

(Buch.-Ham.) Wall. ex G.

Shrub

Apocynaceae

Ban Khirro, Khuria, Anley Khirrn, Madhese Khirro (Np); Hat (S)

$100-1500$

43 Ichnocarpus frutescens (L.) R. Climber Br.

44 *Rauvolfia serpentina (L) Benth. Ex Kurz.

$46{ }^{*}$ Acorus calamus L

Apocynaceae

Araceae
Sweet Flag (Eg); Sadakppa (Li); Ruklop (Lp); Bojho (Np); Chhowas (Ri); Vacha (Sn); Chota, Jota (Gr-Mn); Suda, Syued (Sh): Shete (Tm): Tshedak (Sh): Shudag

\section{Silijam (Dz), Bortsl (Sh)}

Man kachui (S); Karkalo (Np).

Pindo (S); Ol (Np)
Plant: Anti cancer and antitumour (Sikkim: Database). Leaves: Used as diuretic (Sikkim: Database). Flowers: Eaten to maintain sugar Lel for diabetic patients (Jhapa: [68]).

Plant: Powder or decoction taken to treat acute diarrhea and dysentery

(Darjeeling: [86]; Sikkim: [11, 85]). Bark: Powdered with black pepper and taken to cure cough, cold, fever, diarrhea, and dysentery (Jhapa: [66]). Juice taken in amoebic dysentery (Sikkim: [11, 85]). Bark and seeds: Useful to treat gastric troubles and have anthelmintic property (Panchthar: [67]). Fruit: Used as medicine (KL Bhutan: [71]). Stem: Bark powder or decoction given to livestock to treat constipation, problems during stool passing and dysentery (Sikkim: [84]).

Leaves: Extraction used in stomach pain (Jhapa: [66]). Stem: Extraction used in toothache and gum swelling (Jhapa: [66]).

Young shoots: Extract given to cure pneumonia (Jhapa: [68]). Roots, stems and leaves: Used to treat fever (Panchthar: [67]). Roots: Extract used as medicine (KL Bhutan: [71]). Useful in insomnia (Sikkim: Database), high blood pressure, and snake bite (Jhapa: [66]; Sikkim: Database). Extract taken in stomach pain and intestinal worms (Jhapa: [66]). Powder administered orally as antidote to snake-bite (Sikkim: [84]).

Fruits: Used as medicine (KL Bhutan: [71]).

Rhizomes: Used in treatment of epilepsy and other mental ailments, chronic diarrhoea (Darjeeling: [86]; Sikkim: [69]), colic pains and as a brain tonic (Sikkim [697]). Used to cure cold, cough (Jhapa: [68]; Sikkim: [11, 79]; Ilam: [73]), sore throat, bronchitis, tonsil, toothache, headache (Jhapa: [68]; Ilam: [73]), bodypain throat, bronchitis, tonsil, toothache, headache (Jhapa: [68]; llam: [7u]), bodypan
(Jhapa: [66]), scabies, sinusitis (Panchthar: [67]) and also as vermifuge and (Jhapa: [66]), scabies, sinusitis (Panchthar: [67]) and also as vermifuge and
antispasmodic (Sikkim: [75]). Used in skin disease (Darjeeling: [80]; Sikkim: [11 antispasmodic (Sikkim: [75]). Used in skin disease (Darjeeling: [80]; Sikkim: [11,
$79,89]$ ), malaria and asthma (Darjeeling: [80, 89]). Juice used as insecticide (Panchthar: [67]; Sikkim: [75]). Decoction taken as antipyretic (Sikkim: [11, 69]; Darjeeling: [78]) and also to treat rheumatism (Darjeeling: [78]). Pounded with the rhizome of Curcuma zedoaria and given in ulcers and abdominal pains (Jhapa: [66]). Extract taken to cure measles (Sikkim: [79]). Crushed and boiled with salt and decoction used to massage for fortnight (Darjeeling: [74]). Paste applied as ointment on wounds in cattle, also administered in indigestion in cattle (Sikkim: [84).

Rhizomes: Used as medicine (KL Bhutan: [71]).

Petioles and tuber: Edible as vegetable (Jhapa: [92]). Young petioles: Cooked with Colocasia esculenta and taken orally in cold and cough (Jhapa: [66]).

Tubers: Edible (KL Bhutan: [70]).

Tubers and petioles: Used as vegetable (Jhapa: [92]).
48 Alocasia macrorrhizos (L) G. Herb

49 Alocasia spp

50 Amorphophallus paeonifolus C.

Li, Y. Shiao \& S. L. Tseng
Araceae

Araceae
$100-800$

100-800 
Table 1 NTFPs used by the local people of the Kangchenjunga Landscape, Eastern Himalaya (Continued)

\begin{tabular}{|c|c|c|c|c|c|c|}
\hline 51 & $\begin{array}{l}\text { Arisaema costatum (Wall.) } \\
\text { Mart. ex Schott }\end{array}$ & Herb & Araceae & $\begin{array}{l}\text { Glatli (Gr); Asek kaki (Li); Dhakayo, } \\
\text { Jangali Makai (Np); Thwa (Sh) }\end{array}$ & $1900-2800$ & $\begin{array}{l}\text { Leaves: Dried leaves boiled and cooked as vegetable (Taplejung: [87]). Young } \\
\text { shoots used to make curry and corn eaten after repeated boiling (llam: [94]). }\end{array}$ \\
\hline 52 & Arisaema griffithii Schott & Herb & Araceae & $\begin{array}{l}\text { Asek Makai, Yakla Khomba (Li); } \\
\text { Dhokayo (Np); Doka Banko (Np-DI); } \\
\text { Thwa (Sh); Thwa (WI) }\end{array}$ & $2400-3500$ & $\begin{array}{l}\text { Leaves: Sundried, stored and consumed as vegetable in winter; dried leaves } \\
\text { are also used to make Sinki (dried fermented leaves) (Sikkim: [98]; Taplejung: } \\
[81,88]) \text {. }\end{array}$ \\
\hline 53 & $\begin{array}{l}\text { Arisaema intermedium } \\
\text { Blume }\end{array}$ & Herb & Araceae & Sarpa Makai (Np) & $2600-3400$ & Roots: Juice or paste taken in ulcer and fever (llam: $[73,77])$. \\
\hline 54 & $\begin{array}{l}\text { Arisaema jacquemontii } \\
\text { Blume }\end{array}$ & Herb & Araceae & Banko (Np) & $2700-4700$ & $\begin{array}{l}\text { Roots: Juice taken orally to treat menstrual disorders, toothache, and pain } \\
\text { (llam: }[73,77]) \text {. }\end{array}$ \\
\hline 55 & Arisaema sp. & Herb & Araceae & Dowo (Dz), Rungenengsae (Sh) & & Bulbs: Used as medicine (KL Bhutan: [71]). \\
\hline 56 & Caladium bicolor (Ait.) Vent. & Herb & Araceae & Dungasaru (S) & & $\begin{array}{l}\text { Rhizomes: Pounded and applied on body to relieve from bodyache (Jhapa: } \\
\text { [66]). }\end{array}$ \\
\hline 57 & $\begin{array}{l}\text { Scindapsus officinalis (Roxb.) } \\
\text { Schott }\end{array}$ & Climber & Araceae & Kammare lahara (Np); Dari jappa (S) & $200-300$ & Fruit: Pounded and applied on rheumatism and bodyache (Jhapa: [66]). \\
\hline 58 & $\begin{array}{l}\text { Typhonium trilobatum (L.) } \\
\text { Schott }\end{array}$ & Herb & Araceae & Nirbish (S) & 450 & Rhizome: Paste applied externally to cure rheumatism (Jhapa: [66]). \\
\hline 59 & Aralia cachemirica Decne. & Shrub & Araliaceae & Dal kabro (Np); Dumbak (Wl) & $2400-4200$ & $\begin{array}{l}\text { Plant: Powder contains high nutrient and generates heat in the body of } \\
\text { animal (Taplejung: [81]). Roots: Used to treat cuts and contraction of muscles } \\
\text { (Panchthar: [67]). }\end{array}$ \\
\hline 60 & $\begin{array}{l}\text { Brassaiopsis hainla (Buch.- } \\
\text { Ham. ex D. Don) Seem. }\end{array}$ & Tree & Araliaceae & Chuletro (Np, Li) & $1000-1900$ & Roots: Administered orally in case of dysentery (Sikkim: [79]). \\
\hline 61 & Hedera nepalensis K. Koch & Shrub & Araliaceae & Ivy (Eg); Dudela (Np) & 2000-3200 & Plant: Juice taken orally as antispasmodic (Sikkim: [85]). \\
\hline 62 & $\begin{array}{l}\text { Helwingia himalaica Hook. } \\
\text { f. \& Thomson ex C. B. } \\
\text { Clarke }\end{array}$ & Shrub & Araliaceae & & $2100-2700$ & Fruits: Enhance fertility (Sikkim: [75]). \\
\hline 63 & $\begin{array}{l}\text { Macropanax undulatus } \\
\text { (Wallich ex G. Don) } \\
\text { Seemann }\end{array}$ & Tree & Araliaceae & Chenday (Np) & $400-2200$ & Bark: Administered against diabetes (Darjeeling: [52]). \\
\hline 64 & $\begin{array}{l}\text { Merrilliopanax alpinus (C. B. } \\
\text { Clarke) C. B. Shang }\end{array}$ & Tree & Araliaceae & & & Young shoots: Useful in gastric disorders (Sikkim: [75]). \\
\hline 65 & $\begin{array}{l}\text { Panax pseudo-ginseng } \\
\text { subsp. himalaicus H. Hara }\end{array}$ & Herb & Araliaceae & Mangan, Panchpattery (Np) & & $\begin{array}{l}\text { Roots: Taken to reduce fever, indigestion, and vomiting; also used as tonic } \\
\text { (Sikkim: [75], Database). }\end{array}$ \\
\hline 66 & Panax pseudo-ginseng Wall. & Herb & Araliaceae & Panchapattey (Np) & & $\begin{array}{l}\text { Roots: Used as medicine (KL Bhutan: [71]). Used as stimulant, combating } \\
\text { general debility, headache, vomiting, expectorant, carminative, tonic, in blood } \\
\text { pressure (Sikkim: [69]) and weakness (Ilam: [76]; Sikkim: [69]). Juice given to } \\
\text { cure liver disorders, colic, fever and menstrual disorders (Darjeeling: [74]). }\end{array}$ \\
\hline 67 & $\begin{array}{l}\text { Pentapanax leschenaultii } \\
\text { (DC.) Seem. }\end{array}$ & Tree & Araliaceae & Chinde (Np) & $1600-3700$ & Leaves:Tender leaves after boiling used as curry (llam: [94]). \\
\hline 68 & Areca catechu L. & Tree & Arecaceae & & & Fruit/seed: Used as medicine (KL Bhutan: [71]). \\
\hline
\end{tabular}


Table 1 NTFPs used by the local people of the Kangchenjunga Landscape, Eastern Himalaya (Continued)

\begin{tabular}{|c|c|c|c|c|c|}
\hline 69 & Borassus flabellifer L. & Herb & Arecaceae & Tari (S) & \\
\hline 70 & $\begin{array}{l}\text { Calamus acanthospathus } \\
\text { Griff. }\end{array}$ & Climber & Arecaceae & Cane (Eng) & \\
\hline 71 & Calamus erectus Roxburgh & Climber & Arecaceae & & \\
\hline 72 & Calamus latifolius Roxb. & Climber & Arecaceae & & 600 \\
\hline 73 & Calamus tenuis Roxb. & Climber & Arecaceae & Cane (Eng) & \\
\hline 74 & Caryota urens $\mathrm{L}$. & Tree & Arecaceae & Rangbhang (Np) & \\
\hline 75 & $\begin{array}{l}\text { Phoenix acaulis Roxb. ex } \\
\text { Buch.-Ham. }\end{array}$ & Tree & Arecaceae & Betgera (Np) & 1400 \\
\hline 76 & Phoenix sylvestris Roxb. & Tree & Arecaceae & Thakal (Np) & $150-1500$ \\
\hline 77 & $\begin{array}{l}\text { Plectocomia himalayana } \\
\text { Griffith }\end{array}$ & Herb & Arecaceae & Patsha (Bhut) & $1500-2500$ \\
\hline 78 & $\begin{array}{l}\text { Aristolochia griffithii Hook.f. } \\
\text { \& Thomson ex Duch. }\end{array}$ & Climber & Aristolochiaceae & & \\
\hline 79 & Aristolochia indica $\mathrm{L}$. & Climber & Aristolochiaceae & Godh (S) & \\
\hline 80 & Asclepias curassavica L. & Herb & Asclepiadaceae & Khorsani Kose Phul (Np) & $700-1500$ \\
\hline 81 & $\begin{array}{l}\text { Calotropis gigantea (L.) } \\
\text { Dryand. }\end{array}$ & Shrub & Asclepiadaceae & $\begin{array}{l}\text { Ankh (Np); Gogando-bimtang (Me); } \\
\text { Akona (S) }\end{array}$ & $100-1000$ \\
\hline 82 & $\begin{array}{l}\text { Hoya longifolia Wall. ex } \\
\text { Wight }\end{array}$ & Shrub & Asclepiadaceae & Wax Plant (Eg) & $1400-2300$ \\
\hline 83 & Marsdenia roylei Wight, & Climber & Asclepiadaceae & Baahuni Lahara (Np) & $1400-2400$ \\
\hline 84 & $\begin{array}{l}\text { Marsdenia tenacissima } \\
\text { Weight \& Arn. }\end{array}$ & Climber & Asclepiadaceae & $\begin{array}{l}\text { Bahuni Lahara, Sunamari (Np), } \\
\text { Kamtiongrik (Lp) }\end{array}$ & \\
\hline 85 & Marsdenia tinctoria R. Br. & Climber & Asclepiadaceae & Kali Lahara, Ryom (Np) & \\
\hline 86 & $\begin{array}{l}\text { Campylandra aurantiaca } \\
\text { Baker }\end{array}$ & Herb & Asparagaceae & Nakima (Np) & $1900-2900$ \\
\hline
\end{tabular}

Flowers: Juice taken to quench the thurst (Jhapa: [66]).

Shoots: Edible (KL Bhutan: [70]). Edible and also used as a substitute for rope, as cable for suspension bridges, for wickerwork, baskets, and containers. Thicker cane used for makingfurniture frames, walking sticks and umbrella handles (KL Bhutan: [100]).

Shoots: Edible (Bhutan: [99])

Shoots: Edible (KL Bhutan: [70]). Leaves: Juice used to cures eye diseases (Sikkim: [1 1])

Shoot: Edible (KL Bhutan: [70]). Used to make household items, such as mats, screens and furnitures (KL Bhutan: [100]).

Stem and buds: Inner core pith and terminal bud consumed as vegetable (llam: [94]).

Fruits: Raw fruits used to make vegetable curry (llam: [94]).

Stem: Soft pith eaten raw (llam: [94]).

Young shoots: Taken as vegetable (Bhutan: [99, 102])

Flowers: Used as medicine (KL Bhutan: [71])

Roots and leaves: Paste applied in stomachache and as an antidote in snake bite and scorpion sting (Jhapa: [66]).

Roots: Used to treat cancer, warts, and snake bite (Panchthar: [67]).

Latex and Leaves: Used in sprain and swelling (Jhapa: [66]; Panchthar: [67]; Sikkim: Database). Roots: Extraction given in fever and applied on chest and abdomin to relieve pains (Jhapa: [66]). Latex: Used as medicine (KL Bhutan: [71]). Applied on burns (Jhapa: [66]) and for ring worm (Jhapa: [68]).

Leaves: Applied on burns (Panchthar: [67]).

Plant: Used for cooling and alternative effect in gonorrhea (Sikkim: Database). Roots, leaves and fruit: Decoction used to relieve burning sensation of the genitals (Sikkim: [1 1]).

Roots: Juice taken daily as purgative (Sikkim: [85]).

Leaves: Juice taken for stomachache (Sikkim: [85])

Roots: Stocks given orally in case of food poisoning (Darjeeling: [52]).

Inflorescence: Powdered and taken with water to relieve body pain (Sikkim [11]). Given in food poisoning (Darjeeling: [74]). Flowers: Used as appetizer and taken in diabetes (Sikkim: [69, 79], Database). Taken as curry (Sikkim: [89]). 
Table 1 NTFPs used by the local people of the Kangchenjunga Landscape, Eastern Himalaya (Continued)

8 Eucomis regia (L.) Aiton

Tupistra nutans Wall. Lindl.

90 Acmella calva (Candolle) R. Herb K. Jansen

91 Ageratina adenophora Herb

92 Ageratum conyzoides $\mathrm{L}$.

Lily (C)

Asparagaceae

Nakima (Np)

Mareti $(\mathrm{Np})$

Banmara (Np)

llamejhar (Np)

Asteraceae

Ajania tibetica (Hook.f. \& Shrub Thomson) Tzvelev
94 Anaphalis adnata Wall. ex Herb DC.

95 Anaphalis triplinervis (Sims) Herb C. B. Clarke

96 Artemisia dubia Wall. ex Herb Besser

97 Artemisia indica Willd.

Herb

Artemisia nilagirica (C.B. (larke) Pamp.

99 Artemisia sieversiana Ehrh.ex Shrub Willd.

100 Artemisia vulgaris L.
Herb
Asteraceae

Asteraceae

Asteraceae

Asteraceae

Buki phul (Np)

Bhukiphul (Np)

Pati, Titepati, Titaypati (Np); Sibuma Sungmara (Ri); Chhaphung (Sh); Khenpa (Tb); Sangsin Khemba (Wl)

Namyohoba (Li); Tuknil (Lp); Titepati (Np); Tompe (Sh)

Titepati (Np)

Asteraceae

Asteraceae
Titepati (Np); Titeypati (Li)
Bulb: Used as medicinal (KL Bhutan: [71])

Flower: Taken as appetizer and in diabetes (Sikkim: Database). Inflorescence: Powdered and taken to relieve from body pain (Sikkim: [11]).

1000-1900 Fruit: Juice taken orally for headache and stomachache [66, 77], toothache (Darjeeling: [74]; Ilam: [73, 77]) and sore mouth (Darjeeling: [74]).

850-2500 Plant: Juice applied on fresh cut (Darjeeling: [74]; Ilam: [73]; Sikkim: [11, 75]) and also taken orally in fever (llam: [73]). Leaves: Taken orally in dysentery (Darjeeling: [52]).

200-2000 Whole plant: Used as antidote (KL Nepal: [53]). Leaves: Juice applied on wound (Sikkim: [11]; Panchthar: [67]) and also used as anthelmintic (Panchthar: [67]). Decoction used as antipyretic (Darjeeling: [78]), and also in diarrhea, dysentery, colic, and flatulence (Sikkim: [11]).Tenders chewed to cure diarrhoea and dysentery. Flowers: chewed to treat throat pain (Sikkim: [79]).

Used as incense (KL Bhutan: [70]).

Leaves: Juice applied on fresh cuts and wounds (llam: $[73,77])$.

Flower: Paste applied regularly to cure skin problems (KL Nepal: [53]).

Whole plant: Used in ritual ceremony (Sikkim: [11]).

Leaves: Used to treat pains and possess anthelmintic properties (Panchthar: [67]).Crushed fresh leaves uesd to open decongestant sinuses and to stop nasal bleeding (Taplejung: [81]; Sikkim: [11], Database). Extract used on cuts and bruises (Sikkim: [11], Database). Supposed to possess detergent effect and used as cleansing agent (Sikkim: Database). Taking bath of leaves juice cures skin allergies and leaves chewed to treat mouth ulcer (Sikkim: [111). Used as deobstruent, antispasmodic, obstructed menses and hysteria (Darjeeling: [80]).

Tender shoots and leaves: Used to treat inflammation (Taplejung: [87]). Leaves and flowers: Juice used to treat injuries (Sikkim: [75]). Juice taken in asthma, gastritis and skin disease (llam: [76, 77]).

Shoots and leaves: Used to cure mouth ulcer; paste applied externally on forehead during dizziness and headache (Darjeeling: [52]).

Whole plant: Used as medicine (KL Bhutan: [71]).

Leaves: Used to treat nose bleeding (Sikkim: $[69,79,91])$ nervous and spasmodic affections, asthma and the disease of the brain (Sikkim: [69]). Tender leaves chewed cures mouth ulcers; crushed leaves mixed with water and taken bath cures skin allergy. Juice used as anti-eech, besides its religious values (Sikkim: [79]). Used as deobstruent, antispasmodic obstructed menses and hysteria (Sikkim: [89]). Crushed and extract applied externally on skes and itching in cattle fresh leaves grounded, sap extracted and used as no treat to stop nose bleeding in cattle (Sikkim: [84]). 
Table 1 NTFPs used by the local people of the Kangchenjunga Landscape, Eastern Himalaya (Continued)

101 Aster neo-elegans Grierson Shrub

102 Aucklandia costus Falconer Herb

103 Bidens pilosa $\mathrm{L}$.

Herb

104 Bidens spp.

Herb

105 Blumea hieraciifolia (D. Don) Herb DC

106 Blumea lacera (Burf. f.) DC. Herb

107 Calendula officinalis $\mathrm{L}$.

Herb

108 Chrysanthemum indicum L.

109 Cirsium sp. Herb

110 Cremanthodium humile Herb Maxim.

111 Eclipta prostrata $(L) L=$

12 Elephantopus scaber L. Herb

113 Erigeron multiradiatus (Lindl. Herb ex DC.) Benth. ex C.B. Clarke

114 Eupatorium adenophorum Herb Spreng.

115 Eupatorium cannabinum L. Herb

116 Eupatorium odoratum L. Herb

117 Gnaphalium affine D. Don Herb

118 Grangea maderaspatana (L.) Herb
Asteraceae

Asteraceae

Asteraceae

Asteraceae

Asteraceae

Asteraceae

Asteraceae

Asteraceae

Asteraceae

Asteraceae

Asteraceae

Asteraceae

Asteraceae

Asteraceae

Asteraceae

Asteraceae

Asteraceae

Asteraceae
Kapisful, Kuth (Np)

Kuro (Li)

Zumphirobu (Sh)

Sahasrabooti (Np)

Gangansu (Me); Rando, Ghar nagharn

50-350

$2600-4400$

Godawari (Li)

$100-2900$

Bhringaraj (Np); Khetkeshari (S)

Sahsra Jari (Np); Dadari (Me)

Banmara (Np)

Banmara (Np)

Aule banmara (Np); Daubanthu (Me)

Pahelo Bukey (Np)

Chot Bhidimyan (S)
Whole plant: Used as medicine (KL Bhutan: [71]).

Plant: Believed to cure bronchitis, vomiting, epilepsy, headache, and hysteria (Sikkim: Database).

Leaves: Juice applied to eyes and ears to reduce pain (Sikkim: [79]). Leaves and roots: Extract used in cut and jaundice (KL Nepal: [53]).

Edible (KL Bhutan: [70]).

Leaves: Dried and taken smell to treat asthma (Darjeeling: [74]).

Roots: Paste sticked on and around swelling region to prevent cutaneous infection (Jhapa: [68]). Decoction given in urinary infections, and also with decoction of Plumeria acuta given in gonorrhoea and spermatorrhoea (Jhapa: [66]).

Leaves and flowers: Used as antiseptic, antifungal, diaphoretic, stimulant antispasmodic and in small pox; also used in healing wounds, ulcers, and burns (Sikkim: Database).

Flowers: Dried flowers chewed during stomachache (Sikkim: [79]).

Whole plant/flowers: Used as medicine (KL Bhutan: [71]).

Whole plant/flowers: Used as medicine (KL Bhutan: [71]).

Roots: Used in treatment of snake and scorpion bite (Jhapa: [66]). Leaves and tender shoots: Used to treat cut and wounds and jaundice (Panchthar: [67]). Leaves: Infusion used in catarrhal (Jhapa: [66]).

Roots: Paste applied on the muscular pain (Jhapa: [68]). Fruits: Used as tonic (Jhapa: [66]).

Flowers: Used as medicine (KL Bhutan: [71])

Plant: Juice applied on fresh cut (Sikkim: [75]; llam: [73]) and also taken in fever (llam: [73])

Stem and Leaves: Extract used on cut and bruises to stop bleeding and infection (Darjeeling: [80]; Sikkim: [11], Database).

Leaves: Juice applied on cut and injury as haemostatic and to check nasa bleeding, extract dropped in nose to cure severe headache (Jhapa: [68]). Extract also used in cuts and wounds (Sikkim: Database).

Plant: Whole plant crushed and given orally to infants suffering from diarrhoea (Darjeeling: [52]).

Aerial parts: Pounded together with Sphaeranthus indicus and taken orally as well as inhaled a few drops to restore consciousness during epileptic fit (Jhapa: [66]) 
Table 1 NTFPs used by the local people of the Kangchenjunga Landscape, Eastern Himalaya (Continued)

\begin{tabular}{|c|c|c|c|c|c|}
\hline 119 & $\begin{array}{l}\text { Inula cappa (Buch.-Ham. ex } \\
\text { D.Don) DC. }\end{array}$ & Shrub & Asteraceae & $\begin{array}{l}\text { Golden Samphire, Sheep's Year (Eg); } \\
\text { Bakhrikane, Gaitihare, Kanpate, Tihare- } \\
\text { Phul (Np); Basita, Machram (Ri). }\end{array}$ & $1000-2500$ \\
\hline 120 & Inula helenium L. & Herb & Asteraceae & & \\
\hline 121 & $\begin{array}{l}\text { Ixeridium gracile (DC.) Pak \& } \\
\text { Kawano }\end{array}$ & Herb & Asteraceae & & \\
\hline 122 & $\begin{array}{l}\text { Leontopodium jacotianum } \\
\text { Beauverd }\end{array}$ & Herb & Asteraceae & $\begin{array}{l}\text { Bhuke Phul, Jhulo (Np-Dl); Tawa Thokar, } \\
\text { Tawa Thokar Yungpa (Tb) }\end{array}$ & $2700-4900$ \\
\hline 123 & $\begin{array}{l}\text { Leontopodium } \\
\text { monocephalum Edgew. }\end{array}$ & Herb & Asteraceae & $\begin{array}{l}\text { Bhuke Phul, Jhulo (Np-Dl); Tawa Thokar } \\
\text { goepa (Tb). }\end{array}$ & $4600-5600$ \\
\hline 124 & Leontopodium sp. & Herb & Asteraceae & & \\
\hline 125 & Lorentea sp. & Herb & Asteraceae & Khainingroo or Rumplung (Sh) & \\
\hline 126 & $\begin{array}{l}\text { Pulicaria insignis Drumm. ex } \\
\text { Dunn }\end{array}$ & Herb & Asteraceae & & \\
\hline 127 & Pulicaria sp. & Herb & Asteraceae & & \\
\hline 128 & $\begin{array}{l}\text { Saussurea costus (Falc.) } \\
\text { Lipsch. }\end{array}$ & Herb & Asteraceae & & \\
\hline 129 & $\begin{array}{l}\text { Saussurea gossypiphora } \\
\text { D.Don }\end{array}$ & Herb & Asteraceae & $\begin{array}{l}\text { Bhutkesh, Kapase Phool (Np-Tp); } \\
\text { Yazembawa (Wl) }\end{array}$ & $3500-5700$ \\
\hline 130 & $\begin{array}{l}\text { Senecio cappa Buch.-Ham. } \\
\text { ex D. Don }\end{array}$ & Herb & Asteraceae & Bakhrakane (Np) & \\
\hline 131 & $\begin{array}{l}\text { Senecio chrysanthemoides } \\
\text { DC. }\end{array}$ & Herb & Asteraceae & & \\
\hline 132 & Sonchus arvensis $\mathrm{L}$. & Herb & Asteraceae & Ban-rayo (Np) & \\
\hline 133 & Sonchus wightianus DC. & Herb & Asteraceae & & \\
\hline 134 & $\begin{array}{l}\text { Soroseris hookeriana } \\
\text { (C.B.Clarke) Stebbins }\end{array}$ & Herb & Asteraceae & & \\
\hline 135 & Sphaeranthus indicus L. & Herb & Asteraceae & Bad Bhidimyan (S) & $200-800$ \\
\hline 136 & Tagetes erecta $\mathrm{L}$. & Herb & Asteraceae & Demal-bhiwar (Me) & $1800-2000$ \\
\hline 137 & Tagetes patula $\mathrm{L}$. & Herb & Asteraceae & Sayapatri (Np) & $900-2000$ \\
\hline 138 & $\begin{array}{l}\text { Tanacetu matkinsonii } \\
\text { (C.B.Clarke) Kitam. }\end{array}$ & Herb & Asteraceae & & \\
\hline 139 & $\begin{array}{l}\text { Tanacetum tatsienense } \\
\text { (Bureau \& Franch.) K. } \\
\text { Bremer \& Humphries }\end{array}$ & Herb & Asteraceae & & \\
\hline 140 & $\begin{array}{l}\text { Taraxacum officinale F.H. } \\
\text { Wigg. }\end{array}$ & Herb & Asteraceae & & \\
\hline
\end{tabular}

Roots: Juice used in fever, indigestion, and other stomach disorders

(Taplejung: [81]).

Roots: Used as medicine (KL Bhutan: [71]).

Whole plant: Used as medicine (KL Bhutan: [71]).

Plant: Used as incense (Taplejung: [81]).

Plant: Used as incense (Taplejung: [81]).

Rhizomes: Used as medicinal (KL Bhutan: [71]).

Leaves: Edible (KL Bhutan: [70]).

Whole plant/flowers:Used as medicinal (KL Bhutan: [71]).

Flowers: Used as medicinal (KL Bhutan: [71]).

Roots: Used as medicine (KL Bhutan: [71]).

Whole plant: Used as medicinal (KL Bhutan: [71]). Fibres: Used for various purposes (Taplejung: [81]).

Roots and leaves: Infusion used in fever and boils (llam: [73, 76]).

Whole plant: Used as medicinal (KL Bhutan: [71]).

Roots: Paste applied to reliefe from toothache (Darjeeling: [74]).

Roots: Taken in jaundice (Sikkim: [75]).

Whole plant: Used as medicine (KL Bhutan: [71]).

Shoots: Paste applied on breast of women to cure swelling and wounds (Jhapa: [66]).

Leaves: Juice drunk to cure pneumania and chest pain (Jhapa: [68]). Flowers: Useful in pneumonia, piles, and jaundice (Panchthar: [67]).

Flowers: Chewed to cure sore throat, cough and mouth ulcer (Sikkim: [79]).

Whole plant: Used as medicinal (KL Bhutan: [71]).

Whole plant/flowers:Used as medicinal (KL Bhutan: [71]).

Whole plant: Used as medicinal (KL Bhutan: [71]). 
Table 1 NTFPs used by the local people of the Kangchenjunga Landscape, Eastern Himalaya (Continued)

sikkimense

Hand.- Mazz.

142 Taraxacum tibetanum Herb Hand.-Mazz.

143 Vernonia anthelmintica (L.) Herb

144 Vernonia cinerea (L.) Less. Herb

145 Waldheimia glabra (Decne.) Herb Regel

146 Impatiens balsamina L. Herb

147 Basella alba L.

148 Begonia picta Sm.

Climber

Herb

Asteraceae

Wakhur (Sh); Tuki Phool (Np);

Khurmang, Wakhur (Tb)

Asteraceae

Khenpa-karpo (Dz/Sh/B)

Asteraceae

Sauraj (S)

Jurishuri (S)

Asteraceae

Asteraceae

Gang Poe (Tb), Ghanga-Setik (WI).

Balsaminaceae

Basellaceae

Begoniaceae

149 Berberis angulosa Wall. ex Shrub Hook. f. \& Thomson

150 Berberis aristata DC.

Berberidaceae

Berberidaceae

151 Berberis asiatica Roxb. ex Chutro, Musa Lede (Np); Chotto (NpDI); Daruharidra, Thakti-Layem (R)

Berberidaceae

Berberidacea

Chutro (Np)

153 Mahonia acanthifolia D.Don Shrub

154 Mahonia napaulensis DC. Shrub

Berberidaceae

Mahonia (Eg); Samlikhe, Samjikhe (Li); Chutro, Jamane Mandro (Np); Khlusa (Ri); Daruharidra, Kanchan (Sn); Kerbe (Tm); Kerpa (WI)

$155 *$ Sinopodophyllum hexandrum (Royle) T.S.Ying

Berberidaceae

Himalayan May Apple (Eg); Balulu, Balugu (Km); Laghu Patgra (Np); Mem Kyarbukung (Lp); Chutro, Musa Lede $(\mathrm{Np})$; Chotto (Np-Dl); Kyerwa, Kyerkar Rasanjan (Sn); Kerpatsang (Dz)

Plant: Used as vegetable. Flowers and leaves: Used as galactagogue for

human and cattle (Taplejung: [87]).

Whole plant: Used as medicinal (KL Bhutan: [71]). Gudruk (Np-Dl); Upala, Bamasisi, Ramasisi (Sh, WI); Wolmose (Tb); Goegabetapi (Sh)

$1200-2000$

100-2300

4100-5400

$1200-1900$

200

$3400-4500$

$1800-3500$ (Km, Tb); Chompairaim (Ri); Daruharidra,

$1200-2500$

Fruits: Pounded and taken to remove intestinal worms and to cure stomachache (Jhapa: [66]).

Leaves: Decoction given in fever (Jhapa: [66]).

Plant: Used as incense (Taplejung: [87]).

Plant: Decoction used to cure burns and urinary problems (llam: [73, 76]).

Young shoots: Used as vegetable (Jhapa: [92]).

Plant: Juice taken in headache and conjunctivitis (llam: [73, 76]). Stalks:

Extracts from stalks used for venereal disease (Sikkim: Database). Fruit: Juice applied as an anti-leech agent (Taplejung: [88]). Shoots and leaves: Used to make pickle and jam (llam: [94]).

Stem: Decoction taken orally in blood dysentery and jaundice (Darjeeling [52]

Roots and bark: Used as medicine (KL Bhutan: [71]).Used in jaundice, malaria, fever, and diarrhea; also used externally to cure eye disease (Sikkim: Database), Leaves, flowers and bark: Used in eye disease, bile disorders, lympy disorder, jaundice, malarian fever, swelling, and dysentery (llam: [73]; Panchthar: [67]). Stem: Extract used for hypoglycemic activities (Sikkim: [96]). Fruit: Eaten raw (llam: [73]; Panchthar: [67]), also used to cure rabies (Sikkim: [75]).

Bark: Decoction used to treat conjunctivitis, eye inflammation, and also used as laxative and tonic (KL Nepal: [83]). Roots: Source of dye (KL Nepal: [83]).Bark and root decoction administered orally in jaundice and fever (Sikkim: [79]). Fruit: Eaten raw (Taplejung: [82, 87]). Fruit and leaves: Juice taken in diarrhoea and dysentery (Sikkim: [79]).

Bark: Use to extract dye (KL Bhutan: [70]).

Stem: Decoction taken to treat blood dysentery, diarrhoea and jaundice (Darjeeling: [52]).

Plant: Used for fencing (Taplejung: [81, 82]). Fruit: Used in the treament of urinary disorders (Taplejung: [81, 82]). Ripe berries eaten raw (Taplejung: [81]). Bark: Juice applied in eyes (Sikkim: [79]). Fruit and bark: Decoction taken to treat dysentery, diarrhoea (llam: [73, 76]; Sikkim: [86]), and urinary disorders (llam: $[73,76]$ ).

$2400-4500$

Plant: Useful for typhoid fever, mental disorder, and plague (Sikkim: Database). Used as medicine (KL Bhutan: [71]). Roots: Used as purgative, hepatic stimulant, bile expellant, bitter tonic and in skin diseses (Sikkim: [69]). Decoction used in ulcer and liver troubles (Taplejung: [81]). Crushed and applied externally on hoof to treat infection; decoction used for cattle to treat indigestion (Sikkim: [84]). Roots and fruit: Used as anticancer remedy (Sikkim: [75]), taken in fever and diarrhea (Taplejung: [81]; Sikkim: [75], Database). Fruit: Ripe fruits eaten raw; used in gynecological diseases, menstrual disorders, kidney disease, skin disease, and cough (Taplejung: [81]). 
Table 1 NTFPs used by the local people of the Kangchenjunga Landscape, Eastern Himalaya (Continued)

$$
\text { ex D. Don }
$$

157 Betula cylindrostachya Tree Lindley

158 Betula utilis D. Don Tree

Betulaceae

Betulaceae

159 Oroxylum indicum (L.) Kurz Tree

160 Stereospermum chelonoides Tree (L. f.) DC.

161 Bombax ceiba L.

162 Heliotropium indicum L. Herb

163 Onosma hookeri C. B. Clarke Herb

164 Brassica campestris L. var Herb cumifolia Roxb.

165 Brassica sp.

166 Capsella bursa-pastoris (L) Herb Medik.

167 Cardamine hirsuta L. Herb

168 Cardamine macrophylla Herb Willd

169 Erysimum hieraciifolium

\section{Boraginaceae Hatisude (S)}

Boraginaceae

Brassicaceae

Brassicaceae

Brassicaceae

Brassicaceae

Brassicaceae

Brassicaceae

Rayo (Np)
100

Laljari (Np); Bemu (Bhu); Muktsi (Sh)

Yoongkar (Dz)

Shepherd's Pursa (Eg); Chamsure Jhar,

Tori Ghans, Tori Jhar (Np):

Chhyamachhyaru (Sh, WI)

Simrayo (Np)

Chhurukpa (Sh, Wl)

Chhasey (Dz): Kharshing (Sh): Phaledo (Np) betel nut (llam: [94]).

Leaves: Buds used as substitute for tea leaves (Sikkim: [72]).

Branch: Used during marriage ceremony (Taplejung: [81]). Bark: Crushed and applied on injuries of cattle (Sikkim: [97]). Boiled and used for cleaning wounds as antiseptic (Sikkim: [79]).

Plant useful in jaundice (Darjeeling: [105]). Roots, bark and fruit: Used in fever, bronchitis, dysentery, and asthma (Sikkim: [85]). Root bark: Improves appetite, taken in vomiting, asthma, and bronchitis (Darjeeling: [80, 89]). Bark and seeds: Powder used to treat dropsy, sprains, asthma, urinary disorders (llam: [73, 76, 77]), high fever and pneumonia (Sikkim: [1 1]). Seeds: Used as medicinal (KL Bhutan: [71]). Endosperms eaten to cure pneumonia (Jhapa: [68] Bark: Powder applied on chronic wounds (Jhapa: [68]) and also used to treat burns, boils (Panchthar: [67]), and diarrhea (Darjeeling: [86]; Panchthar: [67]). Flowers:Usedas medicinal (KL Bhutan: [71]). Edible (Darjeeling: [80]). Flowers: Edible (Darjeeling: [80]; llam: [94]; Sikkim: [89]).

Fruit: Tied as an amulet to cure migrain (Jhapa: [66]).

Roots and bark: Used as emetic (Panchthar: [67]) and also used to treat diarrhea and dysentery (Darjeeling: [86]; Panchthar: [67]; Sikkim: Database). Roots: Decoction given in urinary infection, also with decoction of Plumaria acuta given in gonorrhoea and spermatorrhoea (Jhapa: [66]). Flowers: Used as medicine (KL Bhutan: [71]). Buds cooked as vegetable (Jhapa: [92]). Pickled and eaten twice daily to get relief from diarrhea and dysentery (Jhapa: [68]). Paste applied externally on small pox in children (Sikkim: [79]). Exude used as gum (KL Bhutan: [70]).

Young stem: Used with bulb of onion to cure rabies (Jhapa: [66]].

Roots: Oil used externally as hair tonic (Sikkim: [49]). Used as medicinal (KL. Bhutan: [71]).

Whole plant: Used to make fermented material called 'Gundruk' (Darjeeling: [108]; Sikkim: [101]). Used in fever, indigestion and irritation (llam: [76]).

Seed: Used as medicine (KL Bhutan: [71]).

Plant: Used as green vegetable (Taplejung: [87]). Used as medicine (KL Bhutan: [711). Leaves: Juice used in malarian fever (Darjeeling: [78]).

$500-3000$

Shoot: Extract taken to low blood pressure and in cardiac problems (Darjeeling: [74]; Sikkim: [75]).

Plant: Used as vegetable (Sikkim: [98]; Taplejung: [87])and also made fermented vegetables (North-East India: [101]).

Seed: Used as medicinal (KL Bhutan: [71]). 
Table 1 NTFPs used by the local people of the Kangchenjunga Landscape, Eastern Himalaya (Continued)

\begin{tabular}{|c|c|c|c|c|c|c|}
\hline 170 & Lepidium sativum $\mathrm{L}$. & Herb & Brassicaceae & Chamsur (Np) & 200-3000 & $\begin{array}{l}\text { Plant: Consumed as vegetable; useful in piles, asthma, cough, syphilis and } \\
\text { bodyache (llam: [76]). }\end{array}$ \\
\hline 171 & Malcolmia sp. & Herb & Brassicaceae & & & Whole plant:Used as medicine (KL Bhutan: [71]). \\
\hline 172 & $\begin{array}{l}\text { Nasturtium officinale R. } \\
\text { Brown }\end{array}$ & Herb & Brassicaceae & & & $\begin{array}{l}\text { Aerial parts: Decoction given to relieve body pain; young shoots taken as } \\
\text { salad (Ilam: [76, 94]; Sikkim: [11, 79]). Plant: Juice given in indigestion and } \\
\text { urinary disorder (llam: [76]). }\end{array}$ \\
\hline 173 & Raphanus sativus L. & Herb & Brassicaceae & Mula (Np) & & $\begin{array}{l}\text { Whole plant: Used to make fermented material called 'Sinki' and 'Gundruk' } \\
\text { (Darjeeling: [101, 108]). Used in indigestion, liver and gall bladder troubles, } \\
\text { urinary complaints and ear pain (llam: }[73,76]) \text {. }\end{array}$ \\
\hline 174 & Thlaspi arvense L. & Herb & Brassicaceae & Jay-kha (Dz) & & Whole plant: Used as medicinal (KL Bhutan: [71]). \\
\hline 175 & Canarium sikkimense King & Tree & Burseraceae & Poskar shing (Sh) & & $\begin{array}{l}\text { Exude or resin used as medicine (KL Bhutan: [71]). Used as incense (KL Bhutan: } \\
{[70] \text { ). }}\end{array}$ \\
\hline 176 & Garuga pinnata Roxb. & Tree & Burseraceae & Aule Dabdabe, Dubdabey (Np) & $300-1200$ & $\begin{array}{l}\text { Bark: Root bark used for curing skin disease; juice applied to treat dislocated } \\
\text { bones and to heal wounds (Sikkim: [85]). Fruit: Taken to improve digestion } \\
\text { (Sikkim: [85]) }\end{array}$ \\
\hline 177 & $\begin{array}{l}\text { Codonopsis foetens Hook.f. } \\
\text { \& Thomson }\end{array}$ & Climber & Campanulaceae & Gaytangru (Sh) & & Whole plant: Used as medicine (KL Bhutan: [71]). \\
\hline 178 & Lobelia angulata G. Forst. & Herb & Campanulaceae & & & $\begin{array}{l}\text { Plant: Decoction given to treat throat pain and fever. Tender shoots: Juice } \\
\text { applied externally to treat boils and inflammation (Sikkim: [11]). }\end{array}$ \\
\hline 179 & Lobelia pyramidalis Wall. & Herb & Campanulaceae & Eklebir (Np) & & Leaves and flowers: Used as antispasmodic (Sikkim: [75]). \\
\hline 180 & $\begin{array}{l}\text { Pratia nummularia (Lam.) A. } \\
\text { Br. \& Asch. }\end{array}$ & Herb & Campanulaceae & Lanka Sanay (Np) & $1000-2400$ & Leaves: Juice taken orally in dysentery and tonsillitis (Darjeeling: [74]). \\
\hline 181 & Cannabis sativa $\mathrm{L}$. & Herb & Cannabaceae & Gaja (Np); Gaja (S) & & $\begin{array}{l}\text { Leaves: Infusion taken to cure stomach pain and flatulence (Jhapa: [66]). } \\
\text { Stem: Cut into small pieces and fed to livestock to treat inflammation; small } \\
\text { pieces mixed with fodder to feed cattle as a tonic (Sikkim: [84]). Decoction } \\
\text { given orally to treat severe diarrhoea (Sikkim: [75, 79]). Flowers: Dried flower } \\
\text { paste taken in empty stomach to treat diarrhoea (Darjeeling: [86]). Seeds: } \\
\text { Pounded and taken to relieve body pain (Sikkim: }[75,79]) \text {. }\end{array}$ \\
\hline 182 & Canna indica $\mathrm{L}$. & Herb & Cannaceae & & 900 & $\begin{array}{l}\text { Rhizomes: Edible and taken to treat fever (Sikkim: [1 1]). Extraction given to } \\
\text { cure urinary troubles (Jhapa: [66]). }\end{array}$ \\
\hline 183 & Capparis zeylanica L. & Shrub & Capparaceae & Asaria (S) & 150 & Fruits: Edible (Jhapa: [92]). \\
\hline 184 & $\begin{array}{l}\text { Dipsacus atratus Hook.f. \& } \\
\text { Thomson ex C.B.Clarke }\end{array}$ & Herb & Caprifoliaceae & Yika (Dz), Pinsa (Sh), & & Whole plant: Used as medicine (KL Bhutan: [71]). \\
\hline 185 & $\begin{array}{l}\text { Pterocephalus hookeri } \\
\text { (C.B.Clarke) E.Pritz. }\end{array}$ & Herb & Caprifoliaceae & & & Flowers/whole plant: Used as medicine (KL Bhutan: [71]). \\
\hline 186 & $\begin{array}{l}\text { Viburnum cylindricum } \\
\text { Buch.-Ham. ex D. Don }\end{array}$ & Shrub & Caprifoliaceae & $\begin{array}{l}\text { Arrow wood (Eg); Hanggase (Li); } \\
\text { Gharaghuri, Ghar ghure, Ghode khari } \\
\text { (Np) }\end{array}$ & $1000-2500$ & $\begin{array}{l}\text { Seeds: Oil used to treat burns (Panchthar: [67]) and also used for cooking } \\
\text { purpose (Taplejung: [87]). }\end{array}$ \\
\hline 187 & Carica papaya L. & Herb & Caricaceae & Mewa (Np) & $100-1000$ & $\begin{array}{l}\text { Latex: Mixed with salt and applied to cure ringworm (Jhapa: [66]). Fruits: Eaten } \\
\text { raw and also eaten to cure jaundice (Sikkim: [79]). }\end{array}$ \\
\hline 188 & $\begin{array}{l}\text { Arenaria densissima Wall. ex } \\
\text { Edgew. \& Hook.f. }\end{array}$ & Herb & Caryophyllaceae & & & Whole plant: Used as medicine (KL Bhutan: [71]). \\
\hline
\end{tabular}


Table 1 NTFPs used by the local people of the Kangchenjunga Landscape, Eastern Himalaya (Continued)

189 Drymaria cordata (L.) Willd. Herb

ex. Roem. \& Schult.

190 Drymaria diandra Blume Herb

191 Drymaria villosa Cham. \& Herb Schlecht.

192 Celastrus paniculatus Willd. Shrub

Celastraceae

Abijalo (Np)

Kujur (S)

Caryophyllaceae

Avijalo (Np)

193 Chenopodium album L

Herb

Chenopodiaceae

Bethu Saag (Np)

194 Cleome gynandra L. Herb

195 Garcinia cowa Roxb. Tree

196 Hypericum uralum Buch.- Herb Ham. ex D. Don

197 Mesua ferrea L., Tree

198 Terminalia bellirica (Gaertn.) Tree Roxb.

199 Terminalia chebula Retz_. Tree

\section{Cleomaceae}

Clusiaceae

Clusiaceae

Clusiaceae

Combretaceae

Combretaceae
Junge Phool (Np); Seta kata arak (S)

Egg tree (Eg); Kaphal (Np)

Urillo (Np)

Nageeswari (Np)

Bhaayure (Me); Barro (Np); Lopong (S): Baru (Dz/Sh/T/B)

Silikhaa (Me); Aaru (Dz/Sh)
$700-2000$

50-300

Plant: Burned and inhaled for antipyretic effect (Darjeeling: [78]). Paste useful to treat fever, cold and cough (Darjeling: [78]) also used for dog bites (Sikkim: Database), headache and sore throat (Sikkim: [11]). Paste applied externally on fractured bone and bandaged with the help of cotton cloth; decoction administered to animal to treat mouth ulcer (Sikkim: [84]). Above ground administered to animal to treat mouth ulcer (Sikkim: [84]). Above ground
parts: Steamed and smelled during sinus trouble (Darjeeling: [52, 78, 80]; parts: Steamed and smelled during sinus trouble (Darjeeling: [52, 78, 80];
Sikkim: [11]; Panchthar: [67]). Leaves: Pasted with Urena lobata applied for Sikkim: [1 1]; Panchthar: [67]). Leaves: Pasted with Urena lobata applied for
cutaneous infections (Jhapa: [68]). Useful in diarrhea and dysentery (Panchthar: [67]).

Plant: Juice useful in cough, cold and sinusitis (llam: $[73,76])$ and peptic ulcer (llam: [76]).

Shoots: Given to treat pneumonia and sinusitis (Sikkim: [75]).

Plant: Juice taken in fever (Darjeeling: [78]). Fresh juice cures sores of throat (Darjeeling: [80]; Sikkim: [11]) and lungs (Jhapa: [66]). Bark and oil: Applied externally to treat acute stomach pain (Jhapa: [66]). Shoots: Juice taken to treat gastritis and constipation (Jhapa: [66]). Leaves: Juice used as eye drops to cure eye infection. Paste reduces swelling and applied on wounds to heal (Jhapa: [66]). Given to cattle to treat loss of appetite (Sikkim: [84]). Seeds: Paste applied on the skin allergies and good for gout (Sikkim: [11]).

Plant: Used as appetizer, laxative, and diuretic; also useful in treatment of eye diseases, throat troubles, piles, blood heart, and spleen diseases (Sikkim: Database). Cooked and eaten as vegetable to reduce bodypain especially back pain (Sikkim: [79]). Young shoots: Consumed as vegetable (llam: [94]).
300

$100-1300$

$1200-3600$

400-950

$300-1100$

$150-1100$
Fruit: Sun dried and taken to treat dysentery (Sikkim: [85])

Bark: Juice applied on wound and bruises (Sikkim: [75]). Seeds: Aromatic and stimulant (Sikkim: Database)

Bark: Orally administered in various skin diseases (mostly poxes) and in menstrual disorder (Sikkim: Database). Bark or stem paste applied or taken orally in hydrocele and on wound (Sikkim: [53]).

Fruit: Used as medicine (KL Bhutan: [71]) and incense (KL Bhutan: [70]).

Powder drunk to treat constipation (Jhapa: [68]). Useful in bronchitis (Jhapa: [66]; Sikkim: Database), asthma and respiratory trouble.Decoctionadministere to livestock for treatment of diarrhea and dysentery (Sikkim: [84]). Bark: Used for anemia and leucoderma (Sikkim: Database).

Fruits: Edible (Sikkim: [47]). Used as medicine (KL Bhutan: [71]) and incense (KL Bhutan: [70]). Used as tonic and also to cure eye, heart and bladder diseases (Sikkim: Database). Powder taken to recover from gastric (Jhapa: [68]). Consumed during cough and sore throat and mouth ulcers (Sikkim: [79]), Powder of dried fruits and bark given in diarrhoea (Darjeeling: [86]). Fruit and/ or bark decoction administered with small amounts of rock salt to cattle to treat diarrhea and dysentery (Sikkim: [84]). 
Table 1 NTFPs used by the local people of the Kangchenjunga Landscape, Eastern Himalaya (Continued)

\begin{tabular}{|c|c|c|c|c|c|c|}
\hline 200 & $\begin{array}{l}\text { Terminalia myriocarpa Van } \\
\text { Heurck \& Mull. Arg. }\end{array}$ & Tree & Combretaceae & Pani saaj (Np) & $100-1000$ & Bark: Juice applied externally on cuts and wounds (Sikkim: [79]). \\
\hline 201 & $\begin{array}{l}\text { Terminalia tomentosa Wight } \\
\text { \& Arn. }\end{array}$ & Tree & Combretaceae & & & Bark: Use to extract dye (KL Bhutan: [70]). \\
\hline 202 & Commelina benghalensis $\mathrm{L}$. & Herb & Commelinaceae & Kane Jhar (Np) & $900-1800$ & Leaves: Juice applied to treat conjunctivitis (Darjeeling: [74]). \\
\hline 203 & $\begin{array}{l}\text { Commelina paludosa } \\
\text { Blume. }\end{array}$ & Herb & Commelinaceae & Bhaisen Jhar (Np) & $300-3500$ & Root: Paste applied on boils (Darjeeling: [52]). \\
\hline 204 & Streptolirion volubile Edgew. & Herb & Commelinaceae & & $1500-2400$ & $\begin{array}{l}\text { Leaves: Crushed and applied on wounds of ear, nose and navel (Darjeeling: } \\
\text { [52]). }\end{array}$ \\
\hline 205 & Cuscuta reflexa Roxb. & Climber & Convolvulaceae & $\begin{array}{l}\text { Dodder (Eg); Akash Beli, Akashveli, } \\
\text { Amarbel, Indrabeli (Np); Chamjakhikwa, } \\
\text { Jalisang (Ri); Alakjadi (S) }\end{array}$ & $200-3100$ & $\begin{array}{l}\text { Plant: Decoction used to treat jaundice (Taplejung: [88]; Panchthar: [67]; } \\
\text { Sikkim: [75]; llam: [73, 76]). Infusion taken in diarrhea, bronchitis (Panchthar: } \\
\text { [67]; Ilam: [73, 76]), and also applied externally to treat body ache and skin } \\
\text { infections (Jhapa: [66]). Shoots and seeds: Used to cure cough (Sikkim: [75]). }\end{array}$ \\
\hline 206 & Cuscuta sinensis Lam. & Climber & Convolvulaceae & Bayding/dukpoo-ru (Sh) & & Whole plant: Used as medicine (KL Bhutan: [71]). \\
\hline 207 & Evolvulus alsinoides $\mathrm{L}$. & Herb & Convolvulaceae & Sankha Pushpi (Np) & $550-1100$ & Plant: Used as febrifuge and aphrodisiac (Panchthar: [67]). \\
\hline 208 & $\begin{array}{l}\text { Evolvulus nummularius (L.) } \\
\mathrm{L} .\end{array}$ & Herb & Convolvulaceae & Chhatbatiza (S) & $150-910$ & Plant: Applied on scorpion sting, cut, wounds and burns (Jhapa: [66]). \\
\hline 209 & Ipomoea aquatica Forssk. & Climber & Convolvulaceae & Karmi (S); Lahore pani sag (Np) & $300-1500$ & $\begin{array}{l}\text { Leaves and twigs: Eaten as vegetable (Jhapa: [92]). Stem: Necklace prepared } \\
\text { and put round the neck of jaundice patient (Darjeeling: [105]). }\end{array}$ \\
\hline 210 & $\begin{array}{l}\text { Maianthemum purpureum } \\
\text { (Wall.) LaFrankie }\end{array}$ & Herb & Convolvulaceae & $\begin{array}{l}\text { Khhiringlo, Khirro, Sikari-Sag (Np); Lekh } \\
\text { Daro (Np-DI) }\end{array}$ & $2600-4300$ & Young leaves and tender shoots: Cooked as vegetables (Taplejung: [88]). \\
\hline 211 & $\begin{array}{l}\text { Merremia umbellata subsp. } \\
\text { orientalis (Hallier f.) Ooststr. }\end{array}$ & Herb & Convolvulaceae & & $300-480$ & Stem: Extraction taken to enhance lactation (Jhapa: [66]). \\
\hline 212 & Cornus capitata Wall. & Tree & Cornaceae & PhastilNamimpluse (Sh), Poitsi (Dz) & & Fruits: Edible (KL Bhutan: [70]). \\
\hline 213 & Cornus macrophylla Wall. & Tree & Cornaceae & $\begin{array}{l}\text { ChapoilBaminpa (Sh), Boray poitsi (Sh), } \\
\text { Poitsi (Dz) }\end{array}$ & & Fruits: Edible (KL Bhutan: [70]). \\
\hline 214 & $\begin{array}{l}\text { Griselinia lucida (J.R.Forst. \& } \\
\text { G.Forst.) G.Forst. }\end{array}$ & Shrub & Cornaceae & & & Fruits: Used as medicine (KL Bhutan: [71]). \\
\hline 215 & $\begin{array}{l}\text { Bryophyllum pinnatum } \\
\text { (Lam.) Oken }\end{array}$ & Herb & Crassulaceae & Patharkuchi (Np) & & $\begin{array}{l}\text { Leaves: Paste applied on wound, bruises, swelling and insect bites (Sikkim: } \\
\text { [69]). }\end{array}$ \\
\hline 216 & $\begin{array}{l}\text { Kalanchoe integra (Medikus) } \\
\text { Kuntze }\end{array}$ & Herb & Crassulaceae & Hatnokane (Np) & & Leaves: Juice taken orally as purgative (Sikkim: [85]). \\
\hline 217 & Coccinia grandis (L.) Voigt & Climber & Cucurbitaceae & Gol Kankri (Np); Tilkocha (S) & $200-900$ & Fruit: Unripe fruits used as vegetable (Jhapa: [92]). \\
\hline 218 & Cucurbita pepo L. & Climber & Cucurbitaceae & Pharsi (Li) & & $\begin{array}{l}\text { Seeds: Powdered and taken orally for its vermifuge potency in children } \\
\text { (Sikkim: [79]). Fruit: Used as medicine (KL Bhutan: [71]). }\end{array}$ \\
\hline 219 & $\begin{array}{l}\text { Diplocyclos palmatus (L.) C. } \\
\text { Jeffrey }\end{array}$ & Climber & Cucurbitaceae & Kabubotke (S) & $200-1500$ & Leaves: Decoction used in eye infections (Jhapa: [66]). \\
\hline
\end{tabular}

Terminalia myriocarpa Van Tree

202 Commelina benghalensis L. Herb

Herb
Leaves: Decoction used in eye infections (Jhapa: [66]). 
Table 1 NTFPs used by the local people of the Kangchenjunga Landscape, Eastern Himalaya (Continued)

\begin{tabular}{|c|c|c|c|c|c|c|}
\hline 220 & $\begin{array}{l}\text { Herpetospermum } \\
\text { pedunculosum (Ser.) Baill. }\end{array}$ & Climber & Cucurbitaceae & $\begin{array}{l}\text { Ban Karela (Np); Serkyi Metog (Tb); } \\
\text { Mendok Sepu (Wi) }\end{array}$ & $1500-3600$ & $\begin{array}{l}\text { Fruit: Inner par used in stomachache and to treat bile diseases (Taplejung: } \\
\text { [81]). }\end{array}$ \\
\hline 221 & Momordica charantia L. & Climber & Cucurbitaceae & Tite Karela (Np, Li) & $300-2100$ & $\begin{array}{l}\text { Fruit: Juice taken as blood purifier and also helps to control diabetes (llam: } \\
\text { [76]; Sikkim: [79, 96]) and treat opthalmia and bleeding (Ilam: [76]). Used as } \\
\text { vegetable (Ilam: [76]). Leaves and fruit: Useful in gastric troubles (Darjeeling: } \\
\text { [91]). }\end{array}$ \\
\hline 222 & $\begin{array}{l}\text { Trichosanthes tricuspidata } \\
\text { Lour. }\end{array}$ & Climber & Cucurbitaceae & Indreni (Np) & $1200-2300$ & $\begin{array}{l}\text { Roots and fruit: Extract used to treat gonorrhea, asthma, earache, and } \\
\text { hemicrania (llam: }[73,76,77]) \text {. Roots: Used in lung diseases of cattle. Fruit: } \\
\text { Taken to cure asthma (Sikkim: Database). Leaves: Tender shoots used for } \\
\text { vegetable (llam: [94]). }\end{array}$ \\
\hline 223 & $\begin{array}{l}\text { Daphniphyllum himalense } \\
\text { (Benth.) Mull. Arg. }\end{array}$ & Tree & Daphniphyllaceae & Chandan (Np) & $1200-2500$ & Plant: Plant has religious and aesthetic value (Sikkim: [72]). \\
\hline 224 & Dillenia indica L. & Tree & Dilleniaceae & Ramphal (Np) & $150-250$ & $\begin{array}{l}\text { Fruit: Juice with sugar used as cooling beverage in fever and cough (Sikkim: } \\
\text { [11], Database). Bark and leaves: Taken to cure diarrhea and dysentery } \\
\text { (Sikkim: [11], Database). }\end{array}$ \\
\hline 225 & Dillenia pentagyna Roxb. & Climber & Dilleniaceae & Sahad (S); Tatar (Np) & $150-1500$ & Fruit: Edible (Jhapa: [92]). \\
\hline 226 & Dioscorea alata $\mathrm{L}$. & Climber & Dioscoreaceae & Ghartarul (Np) & $600-1200$ & $\begin{array}{l}\text { Roots: Used in fever, rash and itch, constipation, intestinal worms, leprosy, } \\
\text { piles, and gonorrhoea. (Sikkim: Database). Eaten raw to treat throat pain } \\
\text { (Sikkim: [11]). }\end{array}$ \\
\hline 227 & Dioscorea bulbifera $\mathrm{L}$. & Climber & Dioscoreaceae & $\begin{array}{l}\text { Kaching (Lp); Ban tarul, Gittha, } \\
\text { Kukurtarul (Np); Bengo nari (S); } \\
\text { Tshemakewa(Dz), Borang-Joktang/ } \\
\text { Fantang (Sh) }\end{array}$ & $150-2100$ & $\begin{array}{l}\text { Whole plant: Used as medicine (KL Bhutan: [71]). Tubers: Edible (KL Bhutan: } \\
\text { [70]). Used as aphrodisiac, stomachic, appetizer (Sikkim: Database), tonic and to } \\
\text { cure ulcer (Sikkim: [75]). Boiled and eaten after submerging them whole night } \\
\text { in cold water (Jhapa: [92]; Darjeeling: [80]; Ilam: [94]; Sikkim: [89, 107], Database) } \\
\text { and also used for washing clothes, to kill lice and fish and as contraceptive } \\
\text { pills (Sikkim: Database). }\end{array}$ \\
\hline 228 & $\begin{array}{l}\text { *Dioscorea deltoidea Wall. } \\
\text { ex Griseb. }\end{array}$ & Climber & Dioscoreaceae & $\begin{array}{l}\text { Ban Tarul, Kukur Tarul (Np); Kamanduki } \\
\text { Saplokha (Ri) }\end{array}$ & $450-3100$ & $\begin{array}{l}\text { Tubers: Cooked as vegetable; juice taken as oral contraceptives and also used } \\
\text { in lice problems (llam: [76]; Taplejung: [82]). }\end{array}$ \\
\hline 229 & Dioscorea pentaphylla L. & Climber & Dioscoreaceae & Kusok (Lp); Aser, Bantarul, Bhyakur (Np) & $600-1500$ & $\begin{array}{l}\text { Tubers: Boiled and eaten after submerging them whole night in cold water } \\
\text { (Jhapa: [92]). Boiled and taken orally as anthelmintic and wormifuge especially } \\
\text { against tapeworm (Darjeeling: [52]).Tubers and shoots: Used as tonic and can } \\
\text { also cure swelling (Sikkim: [75]). }\end{array}$ \\
\hline 230 & Shorea robusta Gaertn. & Tree & Dipterocarpaceae & Sal (Np); Sasing (Li) & $150-1500$ & $\begin{array}{l}\text { Used to extract oil (KL Bhutan: [91]). Exude used as incense (KL Bhutan: [70]). } \\
\text { Bark: Paste applied on wound and bone fracture (KL Nepal: [53]). }\end{array}$ \\
\hline 231 & Drosera peltata Thunb. & Herb & Droseraceae & & & Whole plant: Used as medicine (KL Bhutan: [71]). \\
\hline 232 & Diospyros lotus L. & Tree & Ebenaceae & Gundum (Dz), Amdebu (Sh) & & Fruit:Used as medicine (KL Bhutan: [71]). \\
\hline 233 & Diospyros montana Roxb. & Tree & Ebenaceae & Gada tarul (S) & 500 & Fruit: Pulp applied on cracks of feet (Jhapa: [66]). \\
\hline 234 & $\begin{array}{l}\text { Elaeagnus infundibularis } \\
\text { Momiy. }\end{array}$ & Shrub & Elaeagnaceae & $\begin{array}{l}\text { Bastard-Oleaster (Eg); Pirima (Li); } \\
\text { Guenlo, Maldhendo, Madilo (Np); Tikun } \\
\text { (Tm) }\end{array}$ & $1500-2500$ & Fruit: Used to make alcohol (Taplejung: [81]). \\
\hline 235 & Elaeagnus latifolia L. & Tree & Elaeagnaceae & & $700-2300$ & Fruit: Edible (KL Bhutan: [70]; Sikkim: [47]). \\
\hline 236 & $\begin{array}{l}\text { Elaeagnus rhamnoides (L.) } \\
\text { A. Nelson }\end{array}$ & Tree & Elaeagnaceae & & & Fruit: Used as medicine (KL Bhutan: [71]). \\
\hline
\end{tabular}


Table 1 NTFPs used by the local people of the Kangchenjunga Landscape, Eastern Himalaya (Continued)

238 Hippophae tibetana Schlecht.

239 Elaeocarpus sikkimensis Masters

240 Elaeocarpus sphaericus Tre (Gaertn.) K. Schum.

241 Elaeocarpus varunua Buch.- Tree Ham. ex Mast.

242 Agapetes serpens (Wight) Shrub Sleumer

$243{ }^{*}$ Gaultheria fragrantissima Shrub Wall

\begin{tabular}{|c|c|c|c|}
\hline 244 & Gaultheria procumbens L. & Shrub & Ericaceae \\
\hline 45 & Gaultheria sp. & Shrub & Ericaceae \\
\hline 46 & $\begin{array}{l}\text { Gaultheria trichophylla } \\
\text { Royle }\end{array}$ & Shrub & Ericaceae \\
\hline 17 & $\begin{array}{l}\text { Lyonia ovalifolia (Wall.) } \\
\text { Drude }\end{array}$ & Tree & Ericaceae \\
\hline & Pieris formosa (Wall.) D.Don. & Tree & Ericaceae \\
\hline & Rhododendron anthopogon & Shrub & Ericacea \\
\hline
\end{tabular}
D. Don

250 Rhododendron arboreum Tree Smith
Elaeagnaceae

Elaeocarpaceae

Elaeocarpaceae

Elaeocarpaceae

Ericaceae

Ericaceae

Ericaceae

Ericaceae Rangkhilayem (Ri) Dhobang (Sh);
Seabuckthorn (Eg); Bhui Chuk (Np) 3800-4500

$1500-2100$

Rudraksh (Np)

Gasha-thungsey (Sh)

Bandare Khorsani (Np)

Wintergreen (Eg); Singjang,

Singjhangma (Li); Dhasingare, Patpate (Np); Limbuni Phool (Np-Tp); Lamchassi (Ri); Chhyaro (Sh,W

Chanze kam (Dz); Shogshingma shing (Sh)

Ani Gnonzing (Sh, Wi), Sanchanchewa (Tm)

Lyonia (Eg); Tapeba (Li); Angeri (Np); Sangemi Dongbu, Syanggomba (Wi).

Balu (Np); Kekphel (Li)

Fragrant Rhododendron (Eg); Sunpati (Np); Balu (Dz/B/Sh)

Porota (Gr); Tokphekalaphun, Thukphewa (Li); Gurans, Laliguras (Np); Dakbun, Tokse (Ri); Pullasa (Sn); Ladukpa, Sendok Dongbu (Wi)

$1300-3300$

2000-3300

$1400-3600$
Bark and fruit: Useful in lung dieases, skin eruptions, and irritations (Sikkim: Database). Fruit: Edible (Sikkim: [95]; KL Nepal: [83]) and also used to make vinegar (KL Nepal: [83]). Used in toothache, joint pain, liver, lungs, and phlegm diseases, menstrual disorders, dysentery, gum infection, blood disorders, diabetes and intestinal parasities (KL Nepal: [83]). Roots: Fresh root nodules chewed to stop vomitting and also to remove bad smell of mouth (Darjeeling: [49]).

Fruit: Edible and also used to obtain yellow dye (KL Nepal: [83]).

Fruit: Edible (llam: [92]; Sikkim: [95]).

Fruit: Used in vata and kapha disease of head and epileptic fits (Sikkim Database). Edible (Sikkim: [89]). Seed: Paste administered to cure cough (Sikkim: [79])

Fruit: Edible (KL Bhutan: [70]).

Flowers: Edible (Sikkim: [80, 89]).

Leaves: Used as antiseptic (Sikkim: [75]), fodder and in ritual ceremony; warm juice used to treat inflammation and swellings (Taplejung: [87]). Leaves and fruit: Decoction used to treat reheumatism (KL Nepal: [83]; Sikkim: [75]) and worms (KL Nepal: [83]; llam: [73]). Fruit: Ripe fruits are eaten raw (KL Nepal: [87]).

Leaves: Used to extract oil (KL Bhutan: [93]).

Whole plant: Used as incense (KL Bhutan: [70]).

Fruit: Ripe fruits eaten raw (Taplejung: [81]; Darjeeling: [80]; Sikkim: [89]).

Leaves: Dried and used as cigarette wrapper (llam: [81]). Infusion taken to treat scabies and dog bite (llam: $[73,76])$.

Roots: Dust applied to treat rheumatism (KL Nepal: [53]).

Aerial parts: Used as incense and snuffed to induce sneezing (Sikkim: Database). Leaves and flowers: Used for stomach, liver, and lung disorders, indigestion, sore throat, and as appetizer and in vomiting (Darjeeling: [98]; KL Nepal: [83]). Used as incense (Sikkim: Database). Flowers: Used as medicine (K Bhutan: [71]). Used to cure blood dysentery (Sikkim: [75]). Whole plant: Used as incense (KL Bhutan: [70]).

Flowers and young leaves: Useful in dysentery (Darjeeling: [80]; llam: [76]; Sikkim: [11, 85]; KL Nepal: [83]), diarrhea and headache (llam: [76]; Sikkim: [85]). Flowers: Used to treat headache (Panchthar: [67]). Powder taken to stop bleeding in female. Flower petals clear throat choking due to fish or chicken bone (Darjeeling: [52]; Sikkim: [11]). Used to make alcohol; paste applied around eyes for good sight (Taplejung: [81]). Used to make local wine (Darjeeling: [108]; llam: [94]). Crushed with water and administered to livestock to treat diarrhea and dysentery (Sikkim: [97]). 
Table 1 NTFPs used by the local people of the Kangchenjunga Landscape, Eastern Himalaya (Continued)

\begin{tabular}{|c|c|c|c|c|c|}
\hline 251 & $\begin{array}{l}\text { Rhododendron } \\
\text { campanulatum D. Don }\end{array}$ & Tree & Ericaceae & $\begin{array}{l}\text { Syapu (Gr); Chimal, Nilo Chimal, Seti } \\
\text { Chimal (Np); Kalma (Sh); Takma Singya } \\
\text { (Tb); Khama, Saje Medok (WI). }\end{array}$ & $2800-4400$ \\
\hline 252 & $\begin{array}{l}\text { Rhododendron lepidotum } \\
\text { Wall. ex G. Don }\end{array}$ & Shrub & Ericaceae & Bhale sunpati (Np) & $2100-4700$ \\
\hline 253 & $\begin{array}{l}\text { Rhododendron setosum D. } \\
\text { Don }\end{array}$ & Shrub & Ericaceae & Sunpatay (Np); Sulo (Dz/Sh/B/T) & $3700-5600$ \\
\hline 254 & $\begin{array}{l}\text { Vaccinium gaultheriifolium } \\
\text { (Griff.) Hook. f. ex C. B. } \\
\text { Clarke }\end{array}$ & Shrub & Ericaceae & Chyansi (Np-Tp); Khapusekma (Li) & $1500-2300$ \\
\hline 255 & Antidesma acidum Retz. & Shrub & Euphorbiaceae & Archal (Np) & $150-1200$ \\
\hline 256 & Baccaurea ramiflora Lour. & Tree & Euphorbiaceae & & $250-1300$ \\
\hline 257 & $\begin{array}{l}\text { Baliospermum montanum } \\
\text { (Willd.) Mull. Arg. }\end{array}$ & Shrub & Euphorbiaceae & Danti (S) & $300-910$ \\
\hline 258 & Bischofia javanica Blume & Tree & Euphorbiaceae & Kainjal (Np) & $150-1200$ \\
\hline 259 & Bridelia retusa (L.) A. Juss. & Tree & Euphorbiaceae & Gayo (Li) & $100-1400$ \\
\hline 260 & $\begin{array}{l}\text { Croton roxburghii N. P. } \\
\text { Balakr. }\end{array}$ & Tree & Euphorbiaceae & Guti (S) & $300-750$ \\
\hline 261 & Euphorbia griffithii Hook.f. & Herb & Euphorbiaceae & & \\
\hline 262 & Euphorbia hirta L. & Herb & Euphorbiaceae & $\begin{array}{l}\text { Aankle Jhar (Np); Gofatkhalakhachri } \\
(\mathrm{Me}) ; \text { Sangadare, pusitoa (S) }\end{array}$ & $150-1500$ \\
\hline 263 & $\begin{array}{l}\text { Euphorbia pulcherrima } \\
\text { Willd. ex Klotzsch }\end{array}$ & Shrub & Euphorbiaceae & & $800-1200$ \\
\hline 264 & Euphorbia royleana Boissier & Shrub & Euphorbiaceae & Siundee (Np); Sijau (Me) & $1100-1200$ \\
\hline 265 & $\begin{array}{l}\text { Euphorbia sieboldiana } \\
\text { C.Morren \& Decne. }\end{array}$ & Herb & Euphorbiaceae & & \\
\hline 266 & Euphorbia sikkimensis Boiss. & Herb & Euphorbiaceae & & \\
\hline 267 & $\begin{array}{l}\text { Glochidion lanceolarium } \\
\text { Voigt. }\end{array}$ & Shrub & Euphorbiaceae & Bangikath (Np) & \\
\hline
\end{tabular}

Wood: Dried and infusion taken in fever (Darjeeling: [78]). Leaves: Used to wrap tobacco (Taplejung: [81]). Flowers: Nectar edible (Taplejung: [81]).

Leaves and flowers: Paste used for bile and lung disease, cold, and blood disorders (KL Nepal: [83]). Leaves: Used for incense (KL Nepal: [83]).

Flowers: Used as medicine (KL Bhutan: [71]). Leaves: Local Buddhist uses leaf as incense (Sikkim: [89]). Whole plant: Used as incense (KL Bhutan: [70]).

Fruit: Eaten raw or pickled; juice used to treat diarrhea and dysentery (Taplejung: [81]).

Bark and leaves: Used to treat cholera (Panchthar: [67]). Fruit: Consumed raw (llam: [94)

Fruits: Edible (Sikkim: [95]).

Seeds: Used in gastric disorders, gouts, and rheumatism (Jhapa: [66]).

Leaves: Chewed to cure sore throat (Sikkim: [1 1]). Bark: Juice taken to cure diarrhea (Sikkim: [11]).

Bark: Paste prepared from the bark of Bridelia retusa and Schima wallichiiapplied externally on cuts and wounds (Sikkim: [79]).

Bark: Pounded and mixed with little amount of oil of Varanus sp. and massage on the body to relieve from measles, chicken pox, and boils (Jhapa: [66]). Roots and bark: Used as purgative (Jhapa: [66]).

Roots: Used as medicine (KL Bhutan: [71]).

Plant: Extraction given to lactating mother to increase the milk prodcution. Root: Given to stop vomitting (Jhapa: [66]). Young shoots: Used to treat excessive bleeding during menstruation and also in gonorrhea (Jhapa: [68]), Latex: Applied on pimples and old wounds (Jhapa: [68]) and also to treat warts and cuts (Darjeeling: [74]).

Latex: Applied on toothache (Sikkim: [11]).

Latex: Applied to cure swelling of skin due to cutaneous and sub-cutaneous infection (Jhapa: [68]). Used to cure cuts and stop bleeding, to relieve from earache, cough, and asthma (Sikkim: Database).

Tuber/roots: Used as medicine (KL Bhutan: [71]).

Tubers: Used as medicine (KL Bhutan: [71]).

Bark: Juice to taken in stomach complaints (Sikkim: [85]). 
Table 1 NTFPs used by the local people of the Kangchenjunga Landscape, Eastern Himalaya (Continued)

268 Homonoia riparia Lour. Shrub

269 Jatropha curcas L.

Shrub

Euphorbiaceae

Euphorbiaceae

Euphorbiaceae

270 Mallotus philippinensis Muel. Tre Arg

271 Ricinus communis $\mathrm{L}$.

Shrub

Euphorbiacea

Aadi (Me); Iradam (S); Chamlingshing

$150-2400$

Aanda (Me); Kaden, Hathi-kane, Sjaiwan 500-1200 (Np); Bhernada (S); Ngera-kharshing (Sh)

Sindure (Np); Rora (S)

272 Tragia involucrata L.

273 Abrus precatorius $\mathrm{L}$.

Climber

Climber

Euphorbiaceae

Fabaceae

Ban Sisnu (Np); Sangelsim (S)

Lalgedi (Np); Karmet (S)

400

300-1100

oeja (Dz); Jasenshing (Sh)

Kondru (S)

Arare (Li)

200-1100

Tal Khukuri (Np); Sola (S)

Padke Siris (Np)

200-1300

Fabaceae

278 Albizia julibrissin Durazz. Tree

279 Albizia lebbeck (L.) Benth. Tree

280 Albizia procera (Roxb.) Tree Benth.

281 Astragalus yunnanensis Herb Franch.

282 Atylosia scarabaeoides (L.) Climber Benth. abacea

Harasiris (Np)

Seti Siris (Li)

300-1100

Fabaceae

Fabaceae
Roots: Decoction taken as laxative (Sikkim: [85]).

Latex: Applied to treat toothache and swelling testicules (Jhapa: [68]). Used to stop bleeding from wounds; also applied to treat burns, eczema, ringworm (Sikkim: Database) and scabies (Jhapa: [66]). Bark: Chewed to cure mouth sores (Jhapa: [66]). Seeds: Used as medicine (KL Bhutan: [71]).

Seeds: Dried and powder applied on the wound of cattle (Sikkim: [97]). Given to pigs along-with food to kill intestinal worms, rushed and applied externally to cure wound, injuries and skin infection (Sikkim: [84]). Fruit: Powder of glandular hairs of fruits applied in sores and wounds (Jhapa: [66]). Bark: Used as medicine (KL Bhutan: [71]). Flowers and fruit: Use to extract dye (KL Bhutan: [70]).

Roots: Used for skin diseases. Tied as an amulet on neck of children to stop vomiting (Jhapa: [66]). Leaves: Juice used to cure headache, boils, and dysentery; paste used to cure jaundice (Sikkim: Database). Warmed with mustard oil and massaged on the body of post delivery women to cure body pain (Jhapa: [66]). Seeds: Used as medicine (KL Bhutan: [71]). Endosperm applied as cream on dryness of skin to cure cracking heels (Jhapa: [68]).

Leaves: Paste applied on local swelling of hands and feet ([66]).

Roots and fruits: Extract taken orally for abortion (KL Nepal: [53]) and also taken for tonsil and pneumonia (Sikkim: Database). Roots: Paste usedto treat urinary troubles and skin disease (llam: [76]) and also in cough, cold and menstrual troubles and also applied to cure wounds (Jhapa: [66]). Powder taken to treat urinary troubles and skin disease (llam: [76]). Leaves: Juice taken orally to relieve urinary complaints (Jhapa: [66]). Fruit: Chewed or fresh root juice administered orally during throat pain (Sikkim: [79]).

Stem: Heartwood extract used as medicinal (KL Bhutan: $[70,71])$ and gum (KL Bhutan: [70]). Stem and roots: Use to extract dye (KL Bhutan: [70]).

Root: Paste used in fever, cough and cold, and also applied against snake and scorpion sting (Jhapa: [66]). Stem: Paste with pepper taken orally to cure blood dysentery (Jhapa: [66]). Leaves: Paste applied on sores and itches (Jhapa: [66])

Leaves: Chewed with sugar and cumin during bleeding gums; juice administered orally in indigestion in infants (Sikkim: [79]).

Plant: Juice and cumin seeds used in reducing fever (Jhapa: [66]).

Bark: Extract or paste used to treat dandruff (KL Nepal: [53]).

Leaves and flowers: Used to cure boils, piles, and diarrhea (Sikkim: [75]).

Bark: Crushed into paste and applied on forehead during fever (Sikkim: [79])

Whole plant: Used as medicine (KL Bhutan: [71]).

Plant: Paste orally administered to treat body swelling (Jhapa: [66]). 
Table 1 NTFPs used by the local people of the Kangchenjunga Landscape, Eastern Himalaya (Continued)

283 Bauhinia purpurea L. Tree $\quad$ Fabaceae $\quad$ Tanki $(\mathrm{Np}) \quad 300-1600$

284 Bauhinia semla Wunderlin Tree Fabaceae

285 Bauhinia vahlii Wight \& Arn. Climber Fabaceae

Makrik (Lp); Verla, Baro Lara, Bhorla (Np) 200-1300

288 Butea monosperma (Lam.) Shrub Kuntze

289 Caesalpinia bonduc (L.) Shrub Roxb.

290 Cassia fistula L

Palans (Np): Marup (S); Flamingo tree (Eng)

Sugrong-bithai (Me)

Raj Brichhya (Np); Mukhralaudhi (Me); Noormui Mirfu Baha (S); Dongkoshing

\section{Cassia occidentalis L.}

Thulo Tapre (Np)

292 Cassia sophera L.

293 Cassia tora L.

Fabaceae

294 Crotalaria pallida Aiton Herb

295 Desmodium gangeticum (L.) Shrub DC

296 Desmodium triflorum (L.) Herb

297 Entada phaseoloides (L)
Fabaceae

Fabaceae

Fabaceae

Fabaceae

Fabaceae
Tapre (Np): Chakora (S)

Tapre (Np); Chakora, Bhede deren (S)

Chhinchhinne (Np)

Sano Chameli (Np)

Pangra (Np), Kolokpu-sae (Sh)
$600-2300$

$350-1600$
$150-1200$

$400-500$

Plant used against animal bite;useful as maturant for boils and ebcesses (Sikkim: Database). Roots: Used as carminative (Sikkim: Database, [79]). Paste applied on boils (Sikkim: [79]). Bark: Used to control diarrhea (Sikkim: Database). Flowers: Used as laxative (Sikkim: Database). Leaves: Used as fodder (llam: [73]). Shoots: Used as vegetable (llam: [94])

Exude: Used as gum (KL Bhutan: [70]).

Bark: Useful in skin disease (Sikkim: Database) and diarrhea (Sikkim: Database, [74]). Leaves: Used as demulcent (Sikkim: Database). Paste applied in factured bone (Sikkim: [97]). Seeds: Used as tonic and aphrodisiac, and also given to treat snake bite (Panchthar: [67]; Sikkim: Database). Roasted and consumed (llam: [92]). Seeds and leaves: Given in dysentery and used as laxative (Sikkim: [75]).

Root: Dried root and bark administered orally in diarrhea (Darjeeling: [86]), Decoction given to expel placenta of cattle (Sikkim: [97]). Bark: Juice taken as tonic in toothache (Sikkim: [11]). Paste taken to cure swelling, leprosy, cough, and menstrual disorder (llam: [73, 76]; Sikkim: [79]). Flower: Juice taken to cure dysentery, diarrhea, and stomach pain (Sikkim: Database). Buds taken for skin disease and ulcer, dried buds chewed to treat bleeding piles (Sikkim: [11]). Cooked as curry (Darjeeling: [80]; Ilam: [73, 76]; Sikkim: [89]). Fruit: Used for blood purification (Sikkim: Database).

Seeds: Used as anthelmintic (Panchthar: [67])

Fruits: Used as medicine (KL Bhutan: [71]). Roots: Used in tuberculosis (Jhapa: [66])

Seeds: Fried to black with coconut oil, crushed and paste applied on scalp with the help of cock's feather for baldness (Jhapa: [68]).

$150-1400$

Leaves: Used for treating skin diseases, extraction taken orally taken to purify blood, and also used as laxative (Jhapa: [66]). Fruits: Used as medicine (KL Bhutan: [71]). Used as diuretic, purgative, and laxative (Panchthar: [67]). Used for asthma, diabetes, and eczema (Sikkim: Database). Paste used to treat the whopping cough (Jhapa: [68]).

$200-1400$

Flowers and seeds: Paste applied in minor skin infection and inflammation (Jhapa: [68]).

700-1000 Bark and seeds: Infusion given in diabetes (Jhapa: [66])

450-1300

Used as medicine (KL Bhutan: [71]). Leaves: Extraction applied on ringworm and itch (Jhapa: [66]).

Roots: Juice drunk to cure body-swelling problems (Jhapa: [68]).

Roots: Used in snake and scropion bite (Jhapa: [66])

Leaves: Juice consumed to treat diarrhoea and dysentery (Darjeeling: [74]).

Seeds: Used as astringent and emetic (Panchthar: [67]; Sikkim: Database) and also useful to treat dandruff (Panchthar: [67]). Used as medicine (KL Bhutan: [71]). 
Table 1 NTFPs used by the local people of the Kangchenjunga Landscape, Eastern Himalaya (Continued)

\begin{tabular}{|c|c|c|c|c|c|}
\hline 298 & $\begin{array}{l}\text { Entada pursaetha subsp. } \\
\text { sinohimalensis Grierson \& D. } \\
\text { G. Long }\end{array}$ & Climber & Fabaceae & & $400-1500$ \\
\hline 299 & Erythrina arborescens Roxb. & Tree & Fabaceae & Phaledo (Np); Gyesey Kung (L) & $1500-3000$ \\
\hline 300 & Erythrina stricta Roxb. & Tree & Fabaceae & $\begin{array}{l}\text { Phaledo (Np); Chhasey (Dz); Kharshing } \\
\text { (Sh) }\end{array}$ & $1000-1600$ \\
\hline 304 & Erythrina suberosa Roxb. & Tree & Fabaceae & Phaledo (Np); Buru marar (S) & $900-1200$ \\
\hline 302 & $\begin{array}{l}\text { Flemingia macrophylla } \\
\text { (Willd.) Merr. }\end{array}$ & Shrub & Fabaceae & Barvasi (Np) & $700-1700$ \\
\hline 303 & Glycyrrhiza glabra L. & Herb & Fabaceae & Jethimadhu (Np) & \\
\hline 304 & Indigofera sp. & Tree & Fabaceae & & \\
\hline 305 & $\begin{array}{l}\text { Macrotyloma uniflorum } \\
\text { (Lam.) Verdc. }\end{array}$ & Tree & Fabaceae & Gahat (Li) & $450-2800$ \\
\hline 306 & Mimosa pudica L. & Herb & Fabaceae & Lazzawathi (Np); Jhapani (S) & $200-1200$ \\
\hline
\end{tabular}

Bark: Juice applied externally to cure skin disease (Sikkim: [111). Seeds: Paste applied to treat mumps; powder acts as antidandruff agent (Sikkim: [11]).

307 Moghania strobilifera (L.) J. Shrub St.-Hil. ex Kuntze

308 Mucuna macrocarpa Wall. Shrub

309 Mucuna monosperma Wall. Shrub

310 Mucuna pruriens (L.) DC. Herb

311 Oxytropis japonica Maxim. Herb

312 Tamarindus indica L.

Tree

Fabaceae

Fabaceae

Fabaceae

313 Vigna vexillata (L.) A. Rich. Herb

314 Castanopsis hystrix Miq. Tree

315 Castanopsis indica (Roxb.) Tree Miq.
Fagaceae

Fagaceae
Barakaulijhar (Np)

Baldengra (Np, Li)

Kauso (Np); Etka (S)

Jojo (S) Imli, Titri (Np), Titri (Me)

Bir ghangra (S)

Patle Katus (Np)

Katoos (Np)
Leaves and bark: Curative efficar

Bark and leaves: Paste given in rheumatism, fever, asthma, and epilepsy (llam: [73]). Seed: Used as medicine (KL Bhutan: [71]).

Bark: Grinded with bark of Oroxylum indicum and given as antidote (Jhapa: [66])

Plant: Decoction given to cattle to cure blood dysentery (Sikkim: [97]).

Roots: Given in cough, fever, dysentery, and chronic hepatitis (Sikkim: Database).

Leaves: Use to extract dye (KL Bhutan: [70]).

Seeds: Decoction used to remove stone developed in kidney (Sikkim: [79]).

Plant: Juice given to relieve from diarrhoea (llam: [73]; Darjeeling: [86]), dysentery, and in treatment of hydrocele (llam: [73]). Roots and leaves: Paste used in case of piles (Darjeeling: [91]; Sikkim: [11], Database) and kidney problems (Sikkim: Database). Roots: Used in treating asthma, fever, cough dysentery, vaginal and uterine complaint (Sikkim: Database). Paste applied externally to treat boils (Sikkim: [11]). Juice taken to cure epilepsy and sexual externally to treat boils (Sikkin: [11]). Juice takn: Da cur pepilepsy and sexual weakness also used to cure eye troubles (Jhapa: [66]). Powder used to clean
tooth (Darjeeling: [74]).

Roots: Juice taken in indigestion, insomnia and epilepsy (Darjeeling: [74]).

Seeds: Powder taken as anthelmentic (Sikkim: [1 1]).

Seeds: Act as expectorant in cough (Sikkim: [79]).

Leaves: Paste used in boils, blisters, and ulcers (Jhapa: [66]). Roots and seeds: Used as antipyretic and aphrodisiac (Panchthar: [67]). Roots: Used in delirium (Jhapa: [66]). Seeds: Used as medicine (KL Bhutan: [71]).

Whole plant: Used as medicinal (KL Bhutan: [71])

Bark: Decoction given in paralysis, ulcers, and inflammations. Infusion along with bark of Ziziphus mauritiana, Anthocephalus chinensis, Shorea robusta, Streblus asper and black salt given to cure flatulence in cattle (Jhapa: [66]) Fruit: Edible (Jhapa: [92]). Used for cough and blood disorders (Sikkim:

Database). Seeds: Paste eaten to cure stomachache (Jhapa: [68]).

Tubers and seeds: Eaten as vegetable (Jhapa: [68]).

Fruit: Eaten raw (Darjeeling: [80]; llam: [94]; Sikkim: [72, 89]).

Fruit: Roasted and consumed (llam: [94]). 
Table 1 NTFPs used by the local people of the Kangchenjunga Landscape, Eastern Himalaya (Continued)

\begin{tabular}{|c|c|c|c|c|c|}
\hline 316 & $\begin{array}{l}\text { Castanopsis tribuloides (Sm.) } \\
\text { A. DC. }\end{array}$ & Tree & Fagaceae & Musure Katus (Np) & $450-2300$ \\
\hline 317 & Quercus glauca Thunb. & Tree & Fagaceae & Phalat (Np); Yahi (Li) & \\
\hline 318 & $\begin{array}{l}\text { Quercus griffithii Hook.f. \& } \\
\text { Thomson ex Miq. }\end{array}$ & Tree & Fagaceae & Sisi (Dz); Benangshing (Sh) & \\
\hline 319 & $\begin{array}{l}\text { Gynocardia odorata } \\
\text { Roxburgh }\end{array}$ & Tree & Flacourtiaceae & Gantay (Np) & $800-1000$ \\
\hline 320 & Gentiana grandiflora Laxm. & Herb & Gentianaceae & & \\
\hline 321 & $\begin{array}{l}\text { Gentiana stylophora } \\
\text { C.B.Clarke }\end{array}$ & Herb & Gentianaceae & & \\
\hline 322 & Gentiana urnula Harry Sm. & Herb & Gentianaceae & & \\
\hline 323 & $\begin{array}{l}\text { Gentiana veitchiorum } \\
\text { Hemsl. }\end{array}$ & Herb & Gentianaceae & Gangi- Pangenmotok (Dz) & \\
\hline 324 & Halenia elliptica D.Don & Herb & Gentianaceae & & \\
\hline 325 & $\begin{array}{l}\text { Swertia angustifolia Buch. } \\
\text { Ham. ex D. Don }\end{array}$ & Herb & Gentianaceae & Vaale Chirayito (Np) & $600-3300$ \\
\hline 326 & $\begin{array}{l}\text { Swertia bimaculata (Sieb. \& } \\
\text { Zucc) C. B. Clarke }\end{array}$ & Herb & Gentianaceae & Chiraito, Tite (Np) & $900-3700$ \\
\hline 327 & $\begin{array}{l}\text { *Swertia chirayita (Roxb. ex } \\
\text { Fleming) Karsten }\end{array}$ & Herb & Gentianaceae & $\begin{array}{l}\text { Tikta (Km, Sh, Wi); Sungkhinwa (Li); } \\
\text { Chiraito, Chiraita, Tite (Np); Khalu (Nw); } \\
\text { Kuple (Ri); Kirattikta (Sn); Timda (Tm); } \\
\text { Gya-Tig, Tigta (Tb) }\end{array}$ & $1200-3000$ \\
\hline 328 & Swertia multicaulis D. Don & Herb & Gentianaceae & $\begin{array}{l}\text { Sharma Guru (Np, Tm); Sepu Gundum } \\
(\text { Sh, WI) }\end{array}$ & $4000-4900$ \\
\hline 329 & $\begin{array}{l}\text { Swertia nervosa (G. Don) C. } \\
\text { B. Clarke }\end{array}$ & Herb & Gentianaceae & Chiraito, Bhale Chiraito, God Tito (Np) & $700-3000$ \\
\hline 330 & Geranium donianum Sweet & Herb & Geraniaceae & Ragatgeri (Np) & $3200-4800$ \\
\hline 331 & Geranium lambertii Sweet & Herb & Geraniaceae & & \\
\hline 332 & Geranium nepalense Sweet & Herb & Geraniaceae & Bhanda (Np) & \\
\hline
\end{tabular}

Fruit: Edible (Sikkim: [89]).

Leaves: Good ingredients for compost (Sikkim: [89]).

Bark: Paste applied on bone fracture (KL Nepal: [53]).

Seeds: Used as medicine (KL Bhutan: [71]).

Fruit: Used to extract oil (KL Bhutan: [93]). Juice taken or eaten raw in fever (Darjeeling: [78]). Seeds: Seed oil applied on skin diseases and leprosy (Sikkim: [75]), also used for massage purpose for infants (Sikkim: [79]). Ripen seeds roasted and the oil extracted to use in various purposes (llam: [94]).

Whole plant: Used as medicine (KL Bhutan: [71]).

Floral parts: Used as medicine (KL Bhutan: [71]).

Whole plant: Used as medicine (KL Bhutan: [71]).

Whole plant/flowers: Used as medicine (KL Bhutan: [71]).

Whole plant: Used as medicine (KL Bhutan: [71]).

Plant: Extract used to treat fever, cough, and cold (llam: [73]).

Plant: Used to treat diarrhea, dysentery, and fever (Taplejung: [81]).

Plant and seeds: Plant infusion and crushed seeds considered most effective in treating fever (Taplejung: [81]; KL Nepal: [83, 103]; llam: [73, 76]), asthma, cold, and cough (Darjeeling: [80]; Taplejung: [81, 87]; KL Nepal: [83, 103]; Sikkim: [11]; llam: [73, 76]). Whole plant: Used as medicine (KL Bhutan: [71]). Used to treat ulcer, asthma, inflammation, and piles (Darjeeling: [80]). Taken as tonic and also in leucoderma and skin diseases (Darjeeling: $[69,78,80]$ ). Decoction taken to cure cold, cough, diarrhea, and stomachache (Darjeeling: [69]; Sikkim: $[11,79])$. Shoots: Taken in dyspepsia (Sikkim: [75]). Leaves: Mixed with young stem of Achyranthes aspera and stem bark of Phyllanthus emblica and decoction given to cure fever and cholera (Jhapa: [66]).

Roots: Decoction used to treat fever, cough, bodyache, and internal injuries; paste applied to prevent bleeding and infection from cuts and wounds (Taplejung: $[81,82,87,88])$

Roots: Used to treat fever, cough, and cold (Taplejung: [82, 88]).

Plant:Juice consumed in renal complications and dysentery (Darjeeling: [74]

Roots:Used as medicine (KL Bhutan: [71]).

Whole plant: Used as astringent (Sikkim: [75]). 
Table 1 NTFPs used by the local people of the Kangchenjunga Landscape, Eastern Himalaya (Continued)

\begin{tabular}{|c|c|c|c|c|c|}
\hline 333 & $\begin{array}{l}\text { Aeschynanthus parviflorus } \\
\text { (D.Don) Spreng. }\end{array}$ & Shrub & Gesneriaceae & & \\
\hline 334 & $\begin{array}{l}\text { Didymocarpus villosus } \\
\text { D.Don }\end{array}$ & Herb & Gesneriaceae & Kumkum (Np) & $900-240$ \\
\hline 335 & Dichroa febrifuga Lour. & Shrub & Hydrangeaceae & $\begin{array}{l}\text { Aseru, Basauli, Bhahak, Pahare Basak, } \\
\text { Vasak (Np); Khasrte, Patre, Polokamji } \\
\text { (Gr); Kiplisang (Ri), Dharmen (Tm); } \\
\text { Borang-yangshabu (Sh) }\end{array}$ & $900-250$ \\
\hline 336 & Curculigo orchioides Gaertn. & Herb & Hypoxiadaceae & $\begin{array}{l}\text { Kalo musali, Musali Kanda (Np); Gahot } \\
\text { (S) }\end{array}$ & $500-110$ \\
\hline 337 & $\begin{array}{l}\text { Belamcanda chinensis (L.) } \\
\text { Redoute }\end{array}$ & Herb & Iridaceae & Tarware phool (Np) & $100-220$ \\
\hline 338 & Iris decora Wall. & Herb & Iridaceae & & \\
\hline 339 & $\begin{array}{l}\text { Engelhardia spicata Lesch. } \\
\text { ex Blume }\end{array}$ & Tree & Juglandaceae & $\begin{array}{l}\text { Yakpohama (Li), Mauwa (Np), Bokto, } \\
\text { Oksipou (Ri) }\end{array}$ & $400-2$ \\
\hline 340 & *Juglans regia L. & Tree & Juglandaceae & Okhar (Np) & $1000-2$ \\
\hline
\end{tabular}

Roots: Decoction used to treat fever (Darjeeling: [78]).

341 Juglans regia var kamaonia Tree

342 Juncus grisebachi Buchenau

343 Anisomeles indica (L.) Herb Kuntze

344 Clerodendrum infortunatum Shrub L.

345 Clerodendrum viscosum Herb Vent.

346 Clinopodium umbrosum (M.Bieb.) C. Koch
Climber Lamiaceae
Juglandaceae

Himalayan Walnut (Eg); Akhor (Gr): Katutung, Takshing, Koto (Gr-Mn); Khayusin, Khesik (Li); Okhar, Hande Okhar (Np); Khaisi (Ri); Akshotak (Sn)

Juncus (C)

Nirepati, Jhusule, Rato Charpate, Rato Pat (Np); Jinting (Sh)

Chitu (Np)

Rajbeli (Np); Lakhanaat (Me); Varni (S)
Leaves: Smoked for its laxative action (KL Nepal: [53]).

Roots: Used as medicine (KL Bhutan: [71]). Used as tonic (Sikkim: [75]).

Decoction taken to treat malarial fever (llam: [76]; Taplejung: [87]; Sikkim: [91],

Database). Roots and leaves: Decoction taken for cough and fever

(Darjeeling: [78, 80]; llam: [76]; Sikkim: [11, 89]). Fruit: Used as febrifuge (Panchthar: [67]). Ink prepared from berries (Sikkim: [1 1]).

Roots/rhizomes: Taken in stomachache, physical weakness (Jhapa: [66]), jaundice, cholera, and diarrhea (Darjeeling: [49]; Panchthar: [67]). Rhizome paste used against skin complaints, stomach ulcer, white discharge in women and dyspepsia (Darjeeling: [49]). Infusion used in gastritis and piles (Darjeeling: [49]).

Rhizomes: Freshly collected and eaten in stomachache and also taken orally as antidote to food poisoining (Darjeeling: [52]).

Fruits: Used as medicine (KL Bhutan: [71]).

Bark: Used in diarrhea and dysentery; paste used in bone fracture (Taplejung: [88]).

Bark: Used for dye and acts as detergent (Darjeeling: [80]; Sikkim: [89]). Juice taken to get rid from intestinal worms (Darjeeling: [86]; Sikkim: [11, 79]). Stem bark: Decoction taken to cure arthritis, rheumatism, skin diseases and

toothache (Sikkim. [79]). Bark and leaves. Juice used as fish poison (Sikkim: [11]). Leaves: Paste applied on the hoof diseases of cattle (Sikkim: [97]) Fruit: Oil used for headache (Darjeeling: [80]). Used in rheumatism (Darjeeling: [91]). Nuts: Edible (Darjeeling: [80]; llam: [94]; Sikkim: [89]). Shell crushed and obtained black color (Sikkim: [11]).

1200-3000 Bark: Paste used for poisoning fish. Fruit: Edible (KL Bhutan: [70]). Boiled in

water to extract dye. Used to treat pneumonia and wounds (Panchthar: [67]). Seeds: Eaten as tonic or to treat throat pain; hard cover of the fruit applied on Seeds: Eaten as tonic or to treat throat pain; hard cover of the
gout or to treat throat and chest pain (Taplejung: $[87,88]$.

Whole plant: Used as medicine (KL Bhutan: [71]).

Roots: Juice taken to cure fever, soar throat, diarrhea, and dysentery (Taplejung: [88]).

Leaves: Used as anthelminthic, fresh juice used as tonic and febrifuge (Sikkim: Database).

Plant: Infusion boiled in water along with the leaves of Azadirachta indica and bath to treat scabies and skin diseases (Jhapa: [66]). Roots: Paste used as antipoison (Jhapa: [68]). Leaves: Paste used as hair tonic (Jhapa: [66]). Leaf juice consumed to treat leucoderma and hydrophobia (Darjeeling: [74]).

Leaves: Juice used to treat cuts and burns (Panchthar: [67]).Eaten as vegetable to maintain good health (Darjeeling: [74]). 
Table 1 NTFPs used by the local people of the Kangchenjunga Landscape, Eastern Himalaya (Continued)

\begin{tabular}{|c|c|c|c|c|c|}
\hline 347 & $\begin{array}{l}\text { Colebrookea oppositifolia } \\
\text { Sm. }\end{array}$ & Herb & Lamiaceae & Dhursuli (Np); Dhusor (Me) & $250-1700$ \\
\hline 348 & $\begin{array}{l}\text { Elsholtzia blanda (Benth.) } \\
\text { Benth. }\end{array}$ & Herb & Lamiaceae & Jungali Tulsi, Ban Silam (Np) & \\
\hline 349 & $\begin{array}{l}\text { Elsholtzia fruticosa (D.Don) } \\
\text { Rehder }\end{array}$ & Shrub & Lamiaceae & $\begin{array}{l}\text { Chhinik (Np); Aamgora, Kansata (Sh); } \\
\text { Jirug Serpo (Km, Tb); Furmi (WI) }\end{array}$ & $1800-4200$ \\
\hline 350 & Eriophyton wallichii Benth. & Herb & Lamiaceae & & \\
\hline 351 & Hyptis suaveolens (L.) Poit. & Shrub & Lamiaceae & Arridari (S) & $150-1000$ \\
\hline 352 & $\begin{array}{l}\text { Isodon coetsa (Buch.-Ham. } \\
\text { ex D.Don) Kudô }\end{array}$ & Herb & Lamiaceae & Mire (Np) & $600-3400$ \\
\hline 353 & $\begin{array}{l}\text { Leucas cephalotes (Roth) } \\
\text { Spreng. }\end{array}$ & Herb & Lamiaceae & Drona puspi (Np); Gante Jhar (Np) & $150-2400$ \\
\hline 354 & $\begin{array}{l}\text { Leucas indica (L.) R. Br. ex } \\
\text { Vatke }\end{array}$ & Herb & Lamiaceae & $\begin{array}{l}\text { Khaangkareh (Me); Gummi (Np); Durup } \\
\text { (S) }\end{array}$ & $70-1000$ \\
\hline 355 & Leucosceptrum canum Sm. & Herb & Lamiaceae & Cheeongkung (L) & $1000-2600$ \\
\hline 356 & $\begin{array}{l}\text { Melissa axillaris (Benth.) } \\
\text { Bakh. f., Herb }\end{array}$ & & Lamiaceae & Sugandhi (Np) & \\
\hline 357 & Mentha arvensis L. & Herb & Lamiaceae & Padina (Np, Li) & $1200-2000$ \\
\hline 358 & Mentha piperita $\mathrm{L}$. & Herb & Lamiaceae & Pudhina (Np) & \\
\hline 359 & Mentha sp. & Herb & Lamiaceae & & \\
\hline 360 & Ocimum tenuiflorum $\mathrm{L}$. & Herb & Lamiaceae & Tulasipatta (S) & $400-900$ \\
\hline 361 & Orthosiphon incurvus Benth. & Herb & Lamiaceae & Tite (Np) & \\
\hline 362 & Perilla frutescens (L.) Britton & Herb & Lamiaceae & Silam (Np, Li) & $600-2400$ \\
\hline 363 & $\begin{array}{l}\text { Phlomoides rotata (Benth. } \\
\text { ex Hook.f.) Mathiesen }\end{array}$ & Herb & Lamiaceae & & \\
\hline 364 & $\begin{array}{l}\text { Pogostemon amarantoides } \\
\text { Benth. }\end{array}$ & Herb & Lamiaceae & Solomon, Namnam (Bhu) & $900-2100$ \\
\hline
\end{tabular}

Leaves: Paste applied in wounds and inflammation of skin (Jhapa: [68]). Juice Lused to treat cuts and bruises (Panchthar: [67]) Juice taken in dysentery (Sikkim: [79]). Bud extract applied in opthalmic problems (KL Nepal: [53]).

Roots: Powder paste with mustard oil applied on the scabies affected area of cattle (Sikkim: [84]). Leaves: Juice given in diarrhoea (Darjeeling: [74]). Shoots: Taken in gastritis (Sikkim: [75]).

Plant: Religious value (Taplejung: [81]). Leaves and spikes: Used as incense (Taplejung: [81]). Roots: Given against tonsilitis (Darjeeling: [52]).

Whole plant: Used as medicine (KL Bhutan: [71]).

Plant: Paste applied on skin infections (Jhapa: [66]). Leaves: Juice warmed and applied over lice and parasities infected area (Darjeeling: [74]).

Leaves and shoots: Used to treat cuts and wounds (Panchthar: [67]).

Plant: Used to treat pneumonia and wounds (Panchthar: [67]) Leaves: Decoction given in menstrual disorders (KL Nepal: [53]).

Leaves: Crushed with Euphorbia hirta and let to inhale for sinusites and nasa infection (Jhapa: [68]). Decoction massaged on forehead to relieve headache; drops poured in nostril to cure sinusitis and earache; juice taken in asthma, and also applied in geneital organs to cure venereal diseases (Jhapa: [66]).

Roots and leaves: Used for epilepsy and wound (Sikkim: [75]).

Leaves: Juice taken with honey to cure fever (Darjeeling: [78]).

Leaves: Juice given to treat rheumatism, fever, weakness, ulcer, wounds, jaundice, cough, asthma and cuts (llam: [76]). Fresh leaves chewed during gastritis and acidity (Sikkim: [79]).

Plant: Paste taken in bodyache (Darjeeling: [52]). Leaves: Juice, paste or oil taken in painful urination, stomach problems and indigestion (llam: [76]).

Leaves: Used as spices (KL Bhutan: [70]).

Leaves: Leaves are pounded and given with unboiled rice in cough and bronchitis (Jhapa: [66]). Chewed to cure mouth ulcers (Sikkim: [79]). Juice given in cardiopathy, asthma, bronchitis, snake bite, urinary disorders (llam: [76]).

Plant: Juice given to cure tooth decay, diarrhoea, wounds, and cuts (llam: [73, 76]).

Seeds: Dried seeds chewed to cure cough and nausea (Sikkim: [79]).

Whole plant: Used as medicine (KL Bhutan: [71]).

Young leaves: Used as vegetables (Bhutan: [102]). 
Table 1 NTFPs used by the local people of the Kangchenjunga Landscape, Eastern Himalaya (Continued)

\begin{tabular}{|c|c|c|c|c|c|}
\hline 383 & Careya arborea Roxb. & Tree & Lecythidaceae & Khumbhi (S) & $200-600$ \\
\hline 384 & $\begin{array}{l}\text { Leea macrophylla Roxb. ex } \\
\text { Hornem. }\end{array}$ & Shrub & Leeaceae & Galeni, Galoni (Np) & 1500 \\
\hline 385 & Aloe vera (L.) Burm. f. & Herb & Liliaceae & Ghiu kumari (Np) & 1200 \\
\hline
\end{tabular}

Bark: Grounded with cumin seeds and given to treat indigestion and flatulence (Jhapa: [66]).

Root: Used to cure snake bite (Panchthar: [67]). Seeds: Chewed to treat viral fever. Wrapped by cloths and tied around the neck of the children to cure stomach pain (Sikkim: [11]).

Plant: Used for antihypergycemic effect (Sikkim: [96]). Purgative (Sikkim:

Database) and used on burns and skin complaints (Jhapa: [66]; Panchthar: [67]; KL Nepal: [53]; Sikkim: Database, [79]). Leaves: Chewed to cure skin and uterine disorder and jaundice (Jhapa: [68]; Ilam: [73, 76]). Used as stomachic, tonic, purgative and anthelmintic. Juice put on head in high fever to reduce body temperature (Sikkim: [69]).

386 *Asparagus racemosus Herb Liliaceae Willd.
Kurilo (Np); Kedar nari (S); Ngalalkom (Sh); Ngakhacho (Dz)

$300-2200$

Roots: Used as medicine (KL Bhutan: [71]). Useful for diabetes, jaundice and urinary disorder (Sikkim: Database). Extract used in diabetes and tuberculosis (Darjeeling: [52]) and applied externally on bone fracture and joint dislocation (Darjeeling: [52]). Also used as diuretic, demulcent, aphrodisiac, refrigerant, tonic, expectorant, astringent, and appetizer (KL Nepal: [83]; Darjeeling: [52]; Panchthar: [67]). Powder taken to enhance lactation for mothers and also useful in anemia and diarrhoea (llam: [73, 76, 77]). Grounded with root of Musa paradisiaca and drunk to cure gonorrhea; decoction given in fever (Jhapa: [66]). Mixed with hay or grain to feed the cattle for fortnight to increase milkyield (Sikkim: [84]). Paste administered orally in fever, cough and cold. Fruit: Eaten to treat pimples (Sikkim: [79]). Shoots: Cooked as vegetable (Taplejung: [82]; KL Nepal: [83]; Panchthar: [67]).

Mhajari (Np) (Lour.) Merr.

388 Fritillaria cirrhosa D. Don

Liliaceae

389 Fritillaria delavayi Franch.

Herb

390 Gloriosa superba L.

Liliaceae

391 Lilium nepalense D. Don

392 Paris polyphylla Smith

Liliaceae

Liliaceae

393 Polygonatum hookeri Baker

394 Smilax bracteata C. Presl subsp. verruculosa (Merrill) T. Koyama
Herb Liliaceae
Climber Liliaceae
Snake's head fritillary (Eg); Ban lasun, Kakolee (Np)

Tsika (Dz)

Langarey Tarul (Np)

Ban Lasun (Np)

Love Apple (Eg); Satuwa (Gr); Tangma $(\mathrm{Km})$; Satuwa, Tintale Banko (Np): Haimavati (Sn); Natar Dhap (Tm)

Ranye (Km), Pangi Ranye (Tb)

Kukurdine (Np)
700-3000

Roots: Used to treat sprains (Panchthar: [67])

Bulb: Boiled and eaten (KL Nepal: [83]). Used in tuberculosis (Sikkim: [75]) and to treat asthma (KL Nepal: [83]; Sikkim: [75]), bronchitis, and bleeding during

Bulb: Used as medicine (KL Bhutan: [71]).

Roots and flowers: Useful in chronic ulcers, leprosy, piles, and abdominal pains (Sikkim: Database).

Plant: Powder used to flavouring dishes; juice taken as tonic (llam: [73]). Bulb: Used to treat scabies and boils (Panchthar: [67]).

Roots: Used as antidotes (Sikkim: [75]) and in stomachache (Taplejung: [81, 82]) and fever (Taplejung: [81, 82]; Sikkim: [75]). Powder applied to heal wound (Darjeeling: [49]; Taplejung: [81]; KL Nepal: [83]). Infusion taken as tonic and also used in diarrhea, dysentery (KL Nepal: [83]; Ilam: [73]) and fever (Darjeeling: [78]). Infusion taken to treat respiratory disorders and worms (llam: [76]).

\section{0-5000 Roots: Eaten raw (Taplejung: [87]).}

1900 Stem: Used for brushing teeth to cure pyorrhoea and gingivitis (Darjeeling: cough (Sikkim: [75]) 
Table 1 NTFPs used by the local people of the Kangchenjunga Landscape, Eastern Himalaya (Continued)

\begin{tabular}{|c|c|c|c|c|c|}
\hline 395 & $\begin{array}{l}\text { Smilax ovalifolia Roxb. ex. } \\
\text { D. Don }\end{array}$ & Climber & Liliaceae & Kukurdaino (Np) & $200-1000$ \\
\hline 396 & Buddleja asiatica Lour. & Shrub & Loganiaceae & Bhinsenpatee (Np); Pndam (L) & $350-2000$ \\
\hline 397 & $\begin{array}{l}\text { Dendrophthoe falcata (L.f.) } \\
\text { Etting. }\end{array}$ & Herb & Loranthaceae & Mandargon banda (S); Aijeru (Np) & $150-900$ \\
\hline 398 & $\begin{array}{l}\text { Scurrula elata (Edgew.) } \\
\text { Danser }\end{array}$ & Shrub & Loranthaceae & & $1600-2700$ \\
\hline 399 & Viscum articulatum Burm.f. & Shrub & Loranthaceae & $\begin{array}{l}\text { Hadchur (Np); Hadjor, Kathkomjunga } \\
\text { (S) }\end{array}$ & 200-1700 \\
\hline 400 & $\begin{array}{l}\text { Woodfordia fruticosa (L.) } \\
\text { Kurz }\end{array}$ & Shrub & Lythraceae & Dhairo (Np); Icha (S) & 200-1800 \\
\hline 401 & Michelia champaca L. & Tree & Magnoliaceae & Chanpak (Eg); Oulichamp (Np) & \\
\hline 402 & Hibiscus rosa-sinensis $\mathrm{L}$. & Shrub & Malvaceae & Jawa Kusum (Np) & $900-1400$ \\
\hline 403 & Hibiscus sabdariffa L. & Herb & Malvaceae & Bel Chandan (Np); Maharetha (Me) & $1000-1500$ \\
\hline 404 & Malva verticillata $\mathrm{L}$. & Shrub & Malvaceae & Halemetok (Dz) & \\
\hline 405 & Sida acuta Burm. f. & Shrub & Malvaceae & Khareto $(\mathrm{Np})$ & $250-3700$ \\
\hline 406 & Sida cordifolia L. & Shrub & Malvaceae & Bamonmara (Np) & $500-1100$ \\
\hline 407 & Sterculia villosa Roxb. & Tree & Malvaceae & Odal (Np) & \\
\hline 408 & Urena lobata L. & Herb & Malvaceae & Samthai (Me) & $200-1300$ \\
\hline 409 & $\begin{array}{l}\text { Melastoma malabathricum } \\
\mathrm{L} \text {. }\end{array}$ & Shrub & Melastomataceae & Augeri, Chulesi (S \& Np) & 200 \\
\hline 410 & Osbeckia nepalensis Hook. & Herb & Melastomataceae & Angeri, Lattey (Np) & $450-2300$ \\
\hline
\end{tabular}

Roots and fruit: Juice used to cure veneral diseases, rheumatism, and wounds (llam: [73, 76]). Leaves and shoots: Used as curry (llam: [94]).

Stem, leaves and flowers: Used in skin complaints and as abortificant (Sikkim: [75], Database).

Leaves: Paste applied externally to treat skin diseases (Jhapa: [66]).

Leaves: Used for making tea leaf for local suja (Bhutan: [99]). Bark: Paste applied on bone fracture (KL Nepal: [53]).

Plant: Used to treat bone fracture (Jhapa: [66]; Panchthar: [67]); also given in ulcers, epilepsy, muscular pains (Sikkim: Database), injuries, and fracture (Darjeeling: [80]; Sikkim: [11, 79], Database). Stem: Used in body pain, fracture, and fever (llam: [76]: Sikkim: [75]). Paste used in [66]).

Bark: Used for gastric trouble.

Bark and flowers: Used in burns, dysentery, and fracture (KL Nepal: [53]; Panchthar: [67]). Flower: Dried and taken in piles, liver complaints. Honey like secretion consumed (llam: [94]). Leaves: Given to cattle to cure ulcer (Sikkim: [97]). Decoction taken in malarian fever (Jhapa: [66]).

Flowers: Taken for stomachache (llam: [76]; Sikkim: [85]) and as carminative in the treatment of dyspepsia (Sikkim: [85]). Flowers and fruit: Use to extract dye (KL Bhutan: [70]).

Flowers: Young flowers given against tonsillitis. Leaves and flowers: Juice used to cure dandruff and hair problems (Darjeeling: [52]).

Leaves: Paste with leaves of Lawsonia inermis applied on the foot to cure wound caused by muddy water during rainy season (Jhapa: [68]).

Flowers: Used as medicine (KL Bhutan: [71]).

Stem: Paste applied on bone fracture (Darjeeling: [74]).

Roots: Half cup root juice and half-tablespoon sugar candy mixed together is given once daily till cured (Darjeeling: [105]).

Bark: Used to make ropes (KL Bhutan: [100])

Leaves: Paste with aerial part of Drymaria cordata applied to cure skin infection and eczema (Jhapa: [68]).

Leaves: Used in fever; decoction given to cattle (Sikkim: [97]). Fruit: Edible (Jhapa: [92]). Stem and roots: Bark paste applied on wounds and skin disease (Darjeeling: [74]).

410 Osbeckia nepalensis Hook. Herb Melastomataceae Angeri, Lattey (Np)

Roots: Decoction given in urinary complaints and diabetes. Leaves: Extract of young leaves or tender shoots applied on forehead and to treat pneumonia fever and common cold (Darjeeling: [74]). Leaves and flowers: Paste used in foot sores of cattle (Darjeeling: [49]). 
Table 1 NTFPs used by the local people of the Kangchenjunga Landscape, Eastern Himalaya (Continued)

Plant: Used as an antiseptic and febrifuge. Useful in treatment of small pox and as tooth brush, prophylactic for mouth and teeth (Sikkim: Database). Leaves: Cooked with rice and eaten during the name giving ceremany. newly born child. Boiled in water and bath taken with warm water to cure scabies and eczema. Dried leaf power taken as blood purifier ( scabies and eczema. Dred leaf power taken as blood purfer (Jhapa. [92]). Juice taken for blood purification and intestinal worms, also applied externally on wounds, sores, blsters, ans skin diseases (Jhapa. [66]). Fresh/dried leaves chewed to control diabetes (Sikkim: [79, 96]). Bark powder used as insecticid (Sikkim: [79]).

412 Dysoxylum hamiltonii $\quad$ Tree $\quad$ Meliaceae Sipochikang (Np) Blume.

413 Melia azedarach L. Tree

Meliaceae

Bakaina (Np); Bokom Bana (S)

700-1100

414 Toona ciliata M. Roem. Tree

Meliaceae

Tooni (Np); Toon (S)

415 Trichilia connaroides (Wight Tree \& Arn.) Bentv.

416 Cissampelos pareira

Climber

Meliaceae

Aankha Taruwa (Np)

Batulpate (Np); Tejomala (S)

Menispermaceae

$700-2400$

Bark: Decoction taken orally to treat stomachache (Sikkim: [85]).

Roots: Used as astringent, and in biliousness, heart pain, vomiting, and leucoderma. Leaves: Juice taken orally as anthelmintic (Sikkim: [85]). Bark Used as anthelmintic (Jhapa: [66]). Fruit: Used in leprosy (Jhapa: [66]).

200-1700 Bark: Pounded and given to cure toothache (Jhapa: [66]; Sikkim: [79]). Flowers and fruit: Use to extract dye (KL Bhutan: [70]). Fruit: Used for chestpain, fever and measles (Sikkim: [79]).

Leaves: Decoction taken to treat cholera (Sikkim: [85]). Leaves and fruit: Useful to treat cholera (Panchthar: [67]).

Plant: Extract given to treat diarrhea (Jhapa: [66]; Sikkim: [11]), dysentery, urinary disorders, and indigestion (Jhapa: [66]). Roots: Used as antidote; paste taken orally in stomach pain, malarian fever, and cold (Jhapa: [66]). Decoction consumed in empty stomach to cure diabetes (Darjeeling: [74]). Extract given to treat blood in urine (Sikkim: [84]). Leaves: Paste applied on wound and to treat blood in urine (Sikkim: [84]). Leaves: Paste applied on wound and cough, gastric troubles, and sore throat (Panchthar: [67]).

$1000-2500$

Tubers: Powder used in diabetes, tuberculosis, asthma, and fever (Darjeeling: $[49,78])$. Oil used externally in the treatment of leucoderma, leprosy and other skin complaints (Darjeeling: [49]). Stem: Bark used to extract fibres (Darjeeling: [49]). Plant: Useful in jaundice (Darjeeling: [105]).

Tubers: Eaten in jaundice (Sikkim: [75]).

Root: Dipped in water and sprinkled in poultry farm to prevent from birld flu. Leaves: Paste applied on boils for opening (Sikkim: [11]).

Roots: Useful in diabetic. Extract used in menstruation disorders and piles (KL Nepal: [53]). Stem and roots: Infusion given in fever (Darjeeling: [69, 78]). Used to make broom (KL Bhutan: [100]). Leaves: Warmed and wrapped around the fractured and painful joints (Jhapa: [66]). Fruit: Boiled in milk and drunk to get rid from tuberculosis (Darjeeling: [74]). Used as medicine (KL Bhutan: [71]).

Latex: Applied on the boils and on the fractured bone (Sikkim: [79]).

$$
\text { Lam }
$$

Moraceae

Rukh Kathar (Li) ex Roxb

Badahar, Barar (Li)
Latex: Applied on boils and on fractured bone (Sikkim: [79]). Fruit: Edible (KL Bhutan: [70]). Raw fruits used as vegetable and ripen fruits eaten raw (llam: 
Table 1 NTFPs used by the local people of the Kangchenjunga Landscape, Eastern Himalaya (Continued)

\begin{tabular}{|c|c|c|c|c|c|c|}
\hline 423 & Ficus auriculata Lour. & Tree & Moraceae & Nimaro, Nibaro (Np) & $250-1700$ & $\begin{array}{l}\text { Roots and fruit: Chewed in constipation and warts (llam: [73]). Fruit: } \\
\text { Consumed (Ilam: [94]). }\end{array}$ \\
\hline 424 & Ficus benghalensis $\mathrm{L}$. & Herb & Moraceae & Banidare (S); Bar (Np) & $500-1200$ & Fruit: Edible (Jhapa: [92]). Also given in fever (Jhapa: [66]). \\
\hline 425 & $\begin{array}{l}\text { Ficus elastica Roxb. ex } \\
\text { Hornem. }\end{array}$ & Tree & Moraceae & Brongshig (Sh) & & Exude used as gum (KL Bhutan: [70]). \\
\hline 426 & Ficus hirta Vahl & Tree & Moraceae & & 900 & Root: Decoction used to treat food poisoning (Sikkim: [11]). \\
\hline 427 & Ficus hispida L.f. & Tree & Moraceae & Setapodo (S); Khasreto (Np) & $450-1100$ & Fruit: Edible (Jhapa: [92]). \\
\hline 428 & Ficus racemosa L. & Tree & Moraceae & Loa (S); Gular Dumri (Np) & $300 \mathrm{~m}$ & $\begin{array}{l}\text { Fruit: Edible (Jhapa: [92]). Also taken to enhance lactation (Jhapa: [66]). Latex: } \\
\text { Applied on boils and blisters (Jhapa: [66]). }\end{array}$ \\
\hline 429 & Ficus religiosa L. & Tree & Moraceae & Peepal (Np) & $150-1500$ & Latex: Applied on the boils on the tongue (llam: [73]). \\
\hline 430 & $\begin{array}{l}\text { Ficus semicordata Buch.- } \\
\text { Ham. ex Sm. }\end{array}$ & Tree & Moraceae & Khasrey, Khaneu, Khaniu (Np) & $200-2700$ & $\begin{array}{l}\text { Bark and latex: Applied on boils to check infection (Sikkim: [1 1], Database). } \\
\text { Latex: Applied on fresh cut (Panchthar: [67]). Fruit: Edible (Ilam: [94]; Jhapa: } \\
\text { [92]). }\end{array}$ \\
\hline 431 & $\begin{array}{l}\text { Ficus subincisa Buch.-Ham. } \\
\text { ex Sm. }\end{array}$ & Herb & Moraceae & Lute Khaneu (Np) & $400-2400$ & Latex: Freshly collected and applied externally on boils (Darjeeling: [52]). \\
\hline 432 & Ficus virens Dryand. & Tree & Moraceae & Kabra (Np) & 500 & Leaf buds: Young unopened leaf buds boiled and used as pickle (llam: [94]). \\
\hline 433 & Morus alba L. & Tree & Moraceae & & & $\begin{array}{l}\text { Leaves, stem and roots: Contains active phytochemical to lower blood } \\
\text { glucose level (Sikkim: [96]). Leaves: Tender leaves chewed in curing } \\
\text { inflammation of vocal cord and hoarse voice (Sikkim: [11]). Fruit: Edible (KL } \\
\text { Bhutan: [70]; Sikkim: [95]). Used to make jam, jellies and drinks (llam: [94]). }\end{array}$ \\
\hline 434 & Morus australis Poir. & Shrub & Moraceae & Kodaz (S); Kimu (Np) & $900-2400$ & $\begin{array}{l}\text { Bark and leaves: Decoction used to cure sore throat (Sikkim: [1 1]). Fruits: } \\
\text { Edible (KL Bhutan: [70]; Jhapa: [92]; Sikkim: [1 1]). Seeds: Extract applied to treat } \\
\text { foot cracks (Sikkim: [1 1]). }\end{array}$ \\
\hline 435 & Morus macroura Miq. & Tree & Moraceae & $\begin{array}{l}\text { Kimbu (Np); Tshendey (Dz); } \\
\text { Sengdengshing (Sh) }\end{array}$ & & $\begin{array}{l}\text { Stem: Extract from heartwood used as medicine (KL Bhutan: [71]). Fruit: Edible } \\
\text { (KL Bhutan: [70]) }\end{array}$ \\
\hline 436 & Streblus asper Lour. & Tree & Moraceae & Khaksi (Np); Sada (S) & $100-500$ & $\begin{array}{l}\text { Fruit: Edible (Jhapa: [68]). Latex: Used to stop bleeding from freshly cut } \\
\text { wound (Jhapa: [66]). }\end{array}$ \\
\hline 437 & Moringa oleifera Lam. & Tree & Moringaceae & $\begin{array}{l}\text { Munga (S); Sajiwang, Sajana (Np); } \\
\text { Sajanamakharia (Me) }\end{array}$ & $150-1100$ & $\begin{array}{l}\text { Leaves, flowers and fruit: Used as vegetable llam: [94]; Jhapa: [92]). Cooked } \\
\text { vegetable taken to control blood pressure (Jhapa: [68]). Bark: Paste mixed with } \\
\text { black goat's milk and taken to cure tuberculosis (Jhapa: [66]). Stem: Paste used } \\
\text { by women for long hairs (Jhapa: [66]). }\end{array}$ \\
\hline 438 & Musa balbisiana Colla & Herb & Musaceae & Bankera (Np) & $200-600$ & $\begin{array}{l}\text { Fruit: Green fruits used as vegetable after boiling and spath for pickle (llam: } \\
\text { [94]). }\end{array}$ \\
\hline 439 & Musa paradisiaca L. & Herb & Musaceae & Kera $(\mathrm{Np})$ & & $\begin{array}{l}\text { Root bark: Pounded together with stem bark of Mango, Jack fruit, Ziziphus } \\
\text { mauritiana, Shorea robusta and Azadirachta indica and taken to cure diarrhea } \\
\text { and dysentery (Jhapa: [66]). Sap: Taken to cure fever (Sikkim: [1 1]). }\end{array}$ \\
\hline 440 & Musa sp. & Herb & Musaceae & Bankera (Np) & & $\begin{array}{l}\text { Leaves: Used in house construction, roofing, and for making temporary sheds, } \\
\text { also used as fodder. Flowers and fruit: Edible (KL Bhutan: [70, 100]). }\end{array}$ \\
\hline 441 & $\begin{array}{l}\text { Myrica esculenta Buch.- } \\
\text { Ham. ex D. Don }\end{array}$ & Tree & Myricaceae & $\begin{array}{l}\text { Box Myrtle (Eg); Kaphal (Np, Gr); } \\
\text { Katphala (Sn); Lalisa (Lm); Chakchansi, } \\
\text { Jheremsi (Ri) }\end{array}$ & $1200-2300$ & $\begin{array}{l}\text { Bark: Powder mixed with tobacco and snuffed to treat sinusitis (Panchthar: } \\
\text { [67]); mixture also given to livestock to cure worm infestation (Taplejung: [87, } \\
\text { 88]). Paste applied to fix fractured bone and cure internal injuries (Taplejung: } \\
\text { [87, 88]). Paste applied on the chest to get relief from cough and bronchitis } \\
\text { (Ilam: [76]; Sikkim: Database). Fruit: Ripen fruits consumed (Ilam: [73, 94]). }\end{array}$ \\
\hline
\end{tabular}


Table 1 NTFPs used by the local people of the Kangchenjunga Landscape, Eastern Himalaya (Continued)

\begin{tabular}{|c|c|c|c|c|c|}
\hline 442 & Myrica nagi Thunb. & Tree & Myricaceae & Tsutsusey (Sh) & \\
\hline 443 & $\begin{array}{l}\text { Horsfieldia kingii (J. D. } \\
\text { Hooker) Warburg }\end{array}$ & Tree & Myristicaceae & Ramgua (Np) & $300-1200$ \\
\hline 444 & Knema angustifolia Roxb. & Tree & Myristicaceae & Ramguwa (Np) & \\
\hline 445 & Ardisia macrocarpa Wall. & Tree & Myrsinaceae & & $1500-2400$ \\
\hline 446 & Ardisia solanacea Roxb. & Tree & Myrsinaceae & Gulaich (S) & $200-1100$ \\
\hline 447 & Embelia ribes Burm.f. & Shrub & Myrsinaceae & Buibidans (Np) & \\
\hline 448 & $\begin{array}{l}\text { Maesa chisia Buch.-Ham. ex } \\
\text { D. Don }\end{array}$ & Shrub & Myrsinaceae & Bilaune (Np); Purmu Kung (L) & \\
\hline 449 & Eugenia kurzii Duthie & Tree & Myrtaceae & Ambakay (Np) & 500 \\
\hline 450 & Eugenia sp. & Tree & Myrtaceae & Mantsisey (Sh); Nasi or Nyasey (Dz) & \\
\hline 451 & Psidium guajava $\mathrm{L}$. & Tree & Myrtaceae & Amba (Np); Aprisam (S) & $450-1200$ \\
\hline 452 & Syzygium cumini (L.) Skeels & Tree & Myrtaceae & $\begin{array}{l}\text { Sokod (S); Jamun (Np); Jamun (Me), } \\
\text { Bjee (Sz), Dangbelingsae (Sh), Ngasi } \\
\text { (Dz) }\end{array}$ & $300-1200$ \\
\hline 453 & Euryale ferox Saliab. & Herb & Nymphaeaceae & Poraini (S); Maknana (Np) & $3000-4000$ \\
\hline 454 & Fraxinus floribunda Wall. & Tree & Oleaceae & Lankuri (Np); Payjew (L) & \\
\hline 455 & Nyctanthes arbor-tristis L. & Shrub & Oleaceae & Parijat (Np); Badibaha, Jhanti (S) & $200-1200$ \\
\hline 456 & Epilobium angustifolium L. & Herb & Onagraceae & $\begin{array}{l}\text { Rosebay (Eg); Bahankot (Km); Seja (Sh, } \\
\text { Wl); Char Pan, Chu Tsi (Tb) }\end{array}$ & $3300-4000$ \\
\hline 457 & Bulbophyllum affine Lindl. & Herb & Orchidaceae & & \\
\hline 458 & $\begin{array}{l}\text { Calanthe plantaginea } \\
\text { Lindley }\end{array}$ & Herb & Orchidaceae & Wangpeimo (Bhu) & $1800-2500$ \\
\hline 459 & Coelogyne cristata Lindl. & Herb & Orchidaceae & Chaandi gaava (Np) & $1000-2000$ \\
\hline 460 & Coelogyne occultata Hook.f. & Herb & Orchidaceae & Churchurbu (Sh) & \\
\hline
\end{tabular}

Fruit:Used as spices (KL Bhutan: [70]).

Fruit: Used to make pickle (llam: [94]).

Fruit: Powder taken orally to treat diarrhea (Sikkim: [85]).

Fruit: Edible (Sikkim: [47]),

Bark: Macerated with flowers of Hibiscus rosa-sinensis and taken to purify the blood and also to arrest bleeding (Jhapa: [66]).

Seeds: Used as medicine (KL Bhutan: [71]). Powdered with milk and given to dog for anthelmintic efficacies (Sikkim: [84]). Fruit: Crushed and given to pigs to kill tapeworm; also used as appetizer for cattle (Sikkim: [84]).

Roots and leaves: Used as insecticide and anthelmintic (Sikkim: [75]).

Fruit: Pulps consumed (llam: [94]).

Fruit: Edible (KL Bhutan: [70])

Bark: Pounded with bark of mango and given to cure piles (Jhapa: [66]).

Leaves: Tender shoots chewed during cough and sore throat (Sikkim: [79]). Leaves: Tender shoots chewed during cough and sore throat (Sikkim: [79]).
Fruit and bark: Semi ripe fruits and bark eaten to cure diarrhoea (Darjeeling:

[86]).

Bark: Juice drunk in dysentery (Jhapa: [66, 68]) and diarrhea (Jhapa: [66]). Leaves: Extraction massaged on forehead of children for cooling and given orally to increase appetite (Jhapa: [66]). Fruits: Edible (Jhapa: [92]). Used as medicine (KL Bhutan: [71]).

medicine (KL Bhutan: [71]).

Bark: Boiled and applied on gout (Sikkim: Database). Applied on bone fracture (Sikkim: [75]).

Bark: Paste used for dislocated bones. Leaves: Boiled and decanted water taken to control malaria fever. Flowers: Offered to god and goddesses (Sikkim: Database). Leaves and flowers: Pounded with warm water and given to woman to ease expulsion of placenta (Jhapa: [66].

Leaves: Dried and used as tea; given as fodder (Taplejung: $[81,88])$.

Plant: Ornamental value (Bhutan: [99]). Flowers: Edible (Bhutan: [99]).

Plant: Cooked and eaten as vegetable (Bhutan: [102]).

Bulbs: Paste used in sores and boils (llam: [76])

Bulb/stem: Used as medicine (KL Bhutan: [71]).

461 Cymbidium longifolium D. Herb Orchidaceae

2100-2500 
Table 1 NTFPs used by the local people of the Kangchenjunga Landscape, Eastern Himalaya (Continued)

$462{ }^{*}$ Tactylorhiza hatagirea Herb Orchidaceae 2800-4200 (D.Don) Soo

463 Dendrobium aphyllum (Roxb.) C.E.C.Fisch

464 Eulophia dabia (D. Don) Herb Hochr.

465 Gymnadenia crassinervis Herb Finet

466 Ponerorchis chusua (D. Don) Herb Soo

467 Pedicularis anas Maxim. Herb

468 Pedicularis decorissima Diels Herb

469 Pedicularis longiflora Herb Rudolph

470 Pedicularis oederi Vahl Herb

471 Oxalis corniculata L. Herb

472 Pandanus nepalensis St. Shrub John

474 Corydalis chaerophylla DC. Herb

475 Corydalis crispa Prain Herb

476 Corydalis dubia Prain Herb

477 Dicentra scandens (D. Don) Climber Walp.

478 Hypecoum erectum L. Herb Papaveraceae
Nahasihukchok (Li); Panch Aunle (Np): Hathejara (Np-DI); Munjataka (Sn):

Lovha (Sh); Wang Lag, Wangpo Lagpa (Tb)

Orchidaceae

Orchidaceae

Hattipaila (Np)

Orchidaceae

Wanglak (B)

Orchidaceae

Ongri (Sh): Wangla, Marpo, Wanglag Mempa (Tb)

Orobanchaceae

Orobanchaceae

Orobanchaceae

Orobanchaceae

Oxalidaceae

Pandanaceae

Papaveraceae

Thakal (Np); Dhamoi (S)

$150-1400$

Papaveraceae

Papaveraceae

Papaveraceae

Papaveraceae

Chari Amilo (Np); Tandi, chatumarak, Kedumarajan (S)

300-2900
Tubers: Used as tonic and given in dysentery (Sikkim: Database) and chronic

fever (Darjeeling: [787. Sikkim: Database). Decoction used to treat fever,

diabetes (llam: [73,76]) and diarrhoea (llam: [73, 76]. Sikkim: Database). Paste applied on cuts and bruises; extract taken orally to cure body ache (Darjeeling: applied on cuts and brulses, extract taken: $[32,80]$. Paste taken in gastric complains, Jaundice, body ache, bone fracture, shoots: Eaten as vegetable (KL Nepal: [83]). Roots: Used as nervine tonic and aphrodisiac (Sikkim: [69]).

Stem: Used as medicine (KL Bhutan: [71]).

Tubers: Juice taken orally as appetizer (Sikkim: [85]).

Roots: Used as medicine (KL Bhutan: [71])

Tubers: Rosted and eaten (Taplejung: [87]).

Whole plant: Used as medicine (KL Bhutan: [71]).

Whole plant: Used as medicine (KL Bhutan: [71]).

Whole plant: Used as medicine (KL Bhutan: [71]).

Plant used as medicine (KL Bhutan: [71]).

Plant: Juice used to treat indigestion, diarrhoea, piles, anemia, and eye problems (llam: $[73,76])$. Chewed raw as appetizer and also checks boils (Sikkim: [1 1]). Pounded with cumin seeds and taken to cure dysentery and diarrhea. Paste applied on forehead to treat headache. Paste mixed with rhizome of Drymaria quercifolia and applied on bone fracture (Jhapa: [66]). Roots: Used in infamation (Sikkim: [75]). Leaves: Juice taken to cure dysentery (Sikkim: [1 1], Database) and fever, anemia, and for digestion (Sikkim: Database). Fruit: Consumed to cure throat pain (Sikkim: [11]). Leaves: Used as vegetable (llam: [94]; Jhapa: [92]).

Leaves: Young leaves chewed as breath sweetener. Fresh leaves acts as cockroach repellant. Used to make mats, carry bags, fishing bags and thatching purpose (llam: [94]). Fruit: Used to make pickle (llam: [94]).

Leaves: Juice applied on cut, wounds, blisters, and burns (Jhapa: [66]). Latex Used in opthalmic infections (Jhapa: [66]). Roots and leaves: Paste or juice used in skin disease, constipation, cough and fever (llam: [76]).

Fruits: Taken in stomachache (Sikkim: [75]).

Whole plant: Used as medicine (KL Bhutan: [71]).

Whole plant: Used as medicine (KL Bhutan: [71]).

Roots: Crushed and used to stop excessive bleeding in females (Sikkim: [11]).

Whole plant: Used as medicine (KL Bhutan: [71]). 
Table 1 NTFPs used by the local people of the Kangchenjunga Landscape, Eastern Himalaya (Continued)

\begin{tabular}{|c|c|c|c|c|c|}
\hline 479 & $\begin{array}{l}\text { Hypecoum leptocarpum } \\
\text { Hook. f. \& Thomson }\end{array}$ & Herb & Papaveraceae & & \\
\hline 480 & Meconopsis grandis Prain & Herb & Papaveraceae & $\begin{array}{l}\text { Upal Ngongpo (Sh, Tb); Upa Gnono } \\
\text { (Wl) }\end{array}$ & $3000-5200$ \\
\hline 481 & $\begin{array}{l}\text { Meconopsis horridula Hook. } \\
\text { f. \& Thomson }\end{array}$ & Herb & Papaveraceae & & \\
\hline 482 & Meconopsis paniculata Prain & Herb & Papaveraceae & Kheldar, Langur (Np-Dl); Upa Sepu (WI) & $3000-4400$ \\
\hline 483 & $\begin{array}{l}\text { Meconopsis simplicifolia (D. } \\
\text { Don) Walp. }\end{array}$ & Herb & Papaveraceae & Upal Mentook (Bhu) & $3300-4500$ \\
\hline 484 & Passiflora foetida $\mathrm{L}$. & Climber & Passifloraceae & Sano Jhar (Np) & $100-1200$ \\
\hline 485 & Passiflora nepalensis Wall. & Climber & Passifloraceae & Garendal (Np) & \\
\hline 486 & Sesamum orientale L. & Shrub & Pedaliaceae & Siwing $(M e)$ & $600-2400$ \\
\hline 487 & $\begin{array}{l}\text { Phyllanthus acidus (L.) } \\
\text { Skeels }\end{array}$ & Tree & Phyllanthaceae & Atummeral (S); Amala (Np) & \\
\hline 488 & *Phyllanthus emblica L. & Tree & Phyllanthaceae & $\begin{array}{l}\text { Amala (Np); Meral (S); Chhorgengsoi } \\
\text { (Sh) }\end{array}$ & $150-1400$ \\
\hline 489 & Phyllanthus reticulatus Poir. & Shrub & Phyllanthaceae & Smeldam (S) & $400-770$ \\
\hline 490 & Phyllanthus urinaria $\mathrm{L}$. & Herb & Phyllanthaceae & Kanthad (S) & $770-1700$ \\
\hline 491 & Phytolacca acinosa Roxb. & Herb & Phytolaccaceae & $\begin{array}{l}\text { Jaringe Sag, Jarko Sag (Np); } \\
\text { Zalmathangru (Sh) }\end{array}$ & 220 \\
\hline
\end{tabular}

Whole plant: Used as medicine (KL Bhutan: [71]).

Fruit and leaves: Juice taken to cure jaundice, dyspepsia, cough, and asth (Panchthar: [67]; llam: [73, 76]). Fruit: Edible and also used as incense (KL Bhutan: [70]). Used as medicine (KL Bhutan: [71]). Good for burning sensation of heat and urinary discharge, liver complaint, and eye trouble (Sikkim: Database). Edible (Jhapa: [92]; Sikkim: [1 1]) and eaten raw to treat cough, dysentery and diarrhea (Sikkim: [1 1]). Used as tonic (Jhapa: [66]). Used to make pickle (llam: [94]). Flowers and fruit: Use to extract dye (KL Bhutan: [70]).

Plant: Used as fodder for goat (Taplejung: [81, 87]). Seeds: Edible (Taplejung:

Whole plant: Used as medicine (KL Bhutan: [71]).

Young twigs: Used as fodder for goat; young stems eaten raw (Taplejung: [81, 87]).

491). Whole plant: Used as medicine (KL Bhutan: [71]).

Leaves: Infusion consumed to cure insomnia, hysteria, epilepsy and as painkiller (Darjeeling: [74])

Roots: Decoction taken in fever (Darjeeling: [78]).

Leaves: Paste applied on burns and boils (Jhapa: [66]).

Leaves: Paste applied in wounds and sores (Jhapa: [66]).

Roots: Used as medicine (KL Bhutan: [71]). Decoction used in jaundice (Taplejung: [88]). Juice dropped in the nose to cure sinusitis (Sikkim: [79]). Leaves: Effective in high blood pressure (Darjeeling: [74]; Sikkim: [75]). Fresh juice applied on cuts and wounds to stop bleeding and infection (Sikkim: Database). Decoction taken to cure body ache and diarrhea (Sikkim: [11]). Seeds and leaves: Used in indigestion and eye problems (llam: [73, 76]).

Roots: Used as anthelminthic, improves appetite, and abdominal pain (Darjeeling: [80]; Sikkim: [89]). Paste given to treat pneumonia in adults (Jhapa: [68]). Powder given to treat cold (Sikkim: [11]). Fruit: Used as medicine (KL Bhutan: [71]). Taken to cure diarrhea, dysentery, piles, and leprosy (Darjeeling: [80]). Dried unripe fruits used as alternative and tonic. Ripe fruits, aromatic, stomachic and carminative. Infusion of dried fruit taken in cough (Darjeeling: [78]: Sikkim: [11]) and fever (Darjeeling: [78]). Seeds: Powder applied to reduce strains (Darjeeling: [80]).

Fruit: Used as spices and medicine (KL Bhutan: [70]).

493 Piper nigrum L.

494 Hemiphragma heterophyllum Wall.
$2600-4100$ 
Table 1 NTFPs used by the local people of the Kangchenjunga Landscape, Eastern Himalaya (Continued)

\begin{tabular}{|c|c|c|c|c|c|}
\hline 495 & $\begin{array}{l}\text { Picrorhiza kurrooa Royle ex } \\
\text { Benth. }\end{array}$ & Herb & Plantaginaceae & Puti-shing (Dz), Kutki (N) & \\
\hline 496 & $\begin{array}{l}\text { Plantago asiatica subsp. } \\
\text { erosa (Wall.) Z. Yu Li }\end{array}$ & Herb & Plantaginaceae & Isagbul (Np) & 400-3800 \\
\hline 497 & Plantago depressa Willd. & Herb & Plantaginaceae & Tsa-shokum (Sh) & \\
\hline 498 & Plantago major L. & Herb & Plantaginaceae & Jibray Jhar (Np) & \\
\hline 499 & Plumbago zeylanica $\mathrm{L}$. & Shrub & Plumbaginaceae & Chitu (Np); Chitigni (S) & $100-1300$ \\
\hline 500 & $\begin{array}{l}\text { Arundinaria intermedia } \\
\text { Munro }\end{array}$ & Herb & Poaceae & Malingo (Np) & $2000-3000$ \\
\hline 501 & Arundinaria maling Gamble & Herb & Poaceae & Himalayan bamboo (Eg); Malingo (Np) & $1500-3500$ \\
\hline 502 & Arundinaria sp. & Herb & Poaceae & Bans (Np) & \\
\hline 503 & $\begin{array}{l}\text { Bambusa nutans Wall. ex } \\
\text { Munro }\end{array}$ & Herb & Poaceae & Mala Bans (Np) & 700-1700 \\
\hline 504 & Bambusa sp. & Herb & Poaceae & Bans (Np) & \\
\hline 505 & Bambusa tulda Roxb. & Herb & Poaceae & Kanda Bans (Np); Mal Bans (S) & \\
\hline 506 & Coix lachryma-jobi L. & Herb & Poaceae & Jargedi (S); Bhirkraulo (Np) & $900-2100$ \\
\hline 507 & $\begin{array}{l}\text { Cymbopogon flexuosus } \\
\text { (Nees ex Steud.) W.Watson }\end{array}$ & Herb & Poaceae & Solubang (Sh) & \\
\hline 508 & Cynodon dactylon (L.) Pers. & Herb & Poaceae & Dubo (Np); Dubu (S) & $100-2000$ \\
\hline
\end{tabular}

Roots: Used as medicine (KL Bhutan: [71]). Fresh root paste applied to cuts, wounds, bruises, and injuries (Sikkim: [91]).

Leaves: Taken to cure toothache (Sikkim: [75]). Paste applied to treat wounds. Seeds: Powder taken to treat diarrhea and dysentery (Sikkim: [11]).

Whole plant: Used as medicine (KL Bhutan: [71]).

Plant: Decoction taken to cure fever (Darjeeling: [78]). Leaves: Juice consumedin the case of throat pain. Flowers and fruit: Juice applied on cuts and wounds (Darjeeling: [74]). Roots: Paste applied externally on cuts and wounds for quick healing (Sikkim: [84]).

Roots: Used in antifertility (Jhapa: [66]). Used to make traditional yeast or starter called 'Marcha' to make local wine (Darjeeling and Sikkim: [108]). Roasted with potatoand taken orally in jaundice (Darjeeling: [105]).

Plant: Used to make mats, baskets and to construct temporary huts (Sikkim: [89]). Leaves: Used as fodder (llam: [73]). Young shoots: Used as vegetables (llam: [73])

Young shoots: Cooked as vegetable or pickled and eaten (llam: [94]; KL Nepa: [83]). Leaves: Used as fodder (KL Nepal: [83]). Mixed with black pepper and feed with little salt to cattle to treat diarrhea and dysentery (Sikkim: [84]). Roots: Ash mixed with mustard oil and paste applied externally on ringworm in cattles (Sikkim: [84]).

Shoots: Used for fencing and to make food and drink containers, hats arrows and quivers. Young shoots edible (KL Bhutan: [100]). Leaves: Used as fodder (KL Bhutan: [100]).

Plant: Used to support prayer flags by Buddhist (Sikkim: [89]).

Shoots: Edible (KL Bhutan: [70]). Split and woven mats (KL Bhutan: [100]).

Plant: Burnt ash of seedlings applied in leprosy (Jhapa: [66]). Young shoots: Chopped and fermented in tight bamboo or glass bottles to make sour pickle locally called 'Mesu' (Darjeeling: [108]; North-east India: [101]).

Fruit: Juice drunk regularly for a long time to cure tuberculosis (Jhapa: [66])

Leaves: Used to extract essential oil (KL Bhutan: [93]). Extract used as medicine (KL Bhutan: [71]).

Plant: Juice taken orally to cure scabies, cut, wound, epilepsy, piles (llam: [73, 76]), and juice along with garlic and warm mustard oil used to rub on body to relieve bodypain (Jhapa: [66]). Roots: Juice used in piles; paste used to heal cuts and wounds (Sikkim: [11]). Freshly prepared juice taken to cure liver cirrhosis (Darjeeling: [52]). Roots and leaves: Decoction used to cure diarrhea and dysentery (Sikkim: [11]).

Young shoots: Chopped and fermented in tight bamboo or glass bottles to make sour pickle locally called 'Mesu' (Darjeeling: [101, 108]; llam: [94]). Used to make water pipes, water vessels, in house construction and also consumed as vegetable (Sikkim: [89]). 
Table 1 NTFPs used by the local people of the Kangchenjunga Landscape, Eastern Himalaya (Continued)

\begin{tabular}{|c|c|c|c|c|c|c|}
\hline 510 & $\begin{array}{l}\text { Dendrocalamus hookeri } \\
\text { Munro }\end{array}$ & Herb & Poaceae & Chilley Bans (Np) & & Shoots: Used in making baskets and in fencing (Sikkim: [89]). \\
\hline 511 & $\begin{array}{l}\text { Dendrocalamus sericeus } \\
\text { Munro }\end{array}$ & Herb & Poaceae & Bans (Np) & & $\begin{array}{l}\text { Shoots: Used to makedomestic and agricultural implements, such as water } \\
\text { containers, baskets, trays, mats, etc. Young shoots edible (KL Bhutan: [100]). } \\
\text { Leaves: Used as fodder (KL Bhutan: [100]). }\end{array}$ \\
\hline 512 & $\begin{array}{l}\text { Desmostachya bipinnata (L.) } \\
\text { Stapf }\end{array}$ & Herb & Poaceae & Kush (Np) & $170-500$ & Roots: Astringent and galactagogue (Panchthar: [67]). \\
\hline 513 & Drepanostachyum sp. & Herb & Poaceae & Bans (Np) & & $\begin{array}{l}\text { Shoots: Used to makebaskets, trays, mats, sieves, livestock shelters and } \\
\text { temporary dwellings. Yound shoots edible (KL Bhutan: [100]). }\end{array}$ \\
\hline 514 & $\begin{array}{l}\text { Eleusine coracana }(\mathrm{L} .) \\
\text { Gaertn. }\end{array}$ & Herb & Poaceae & Kodo (Np) & $1100-2200$ & Seeds: Used to make local wine called 'Jnard' (Darjeeling: [108]). \\
\hline 515 & $\begin{array}{l}\text { Eulaliopsis binata (Retz.) C.E. } \\
\text { Hubb. }\end{array}$ & Herb & Poaceae & Babiyo (Np) & $2000-1000$ & Plant: Whole plant dust applied to treat skin allergy (KL Nepal: [53]). \\
\hline 516 & Fargesia grossa T. P. Yi & Herb & Poaceae & & & Culms: Used to make mats, bags and baskets (Bhutan: [99]). \\
\hline 517 & $\begin{array}{l}\text { Himalayacalamus } \\
\text { hookerianus (Munro) } \\
\text { Stapleton }\end{array}$ & Herb & Poaceae & Pareng (Np) & & $\begin{array}{l}\text { Plant: Used to make basket and hut construction (Darjeeling: [89]). Young } \\
\text { shoots: Consumed as vegetable (Darjeeling: [89]). }\end{array}$ \\
\hline 518 & $\begin{array}{l}\text { Imperata cylindrica (L.) P. } \\
\text { Beauvois }\end{array}$ & Herb & Poaceae & Siru (Np) & $200-1200$ & $\begin{array}{l}\text { Roots: Juice taken in diarrhoea and dysentery (llam: [73]), taken during cough, } \\
\text { cold and fever (Darjeeling: [91]; Sikkim: [79]). A piece of root tied in hair to ease } \\
\text { delivery (Jhapa: [66]). Paste applied on boils (Darjeeling: [91]; Sikkim: [79]). }\end{array}$ \\
\hline 519 & $\begin{array}{l}\text { Phyllostachys edulis } \\
\text { (Carrière) J. Houzeau }\end{array}$ & Herb & Poaceae & Kattabans (Np) & $1000-1600$ & Shoots: Young shoots used to make curry and pickles (Ilam: [94]). \\
\hline 520 & Saccharum spontaneum L. & Herb & Poaceae & Kash (Np); Kashim (S) & & $\begin{array}{l}\text { Roots: Extract taken to kill intestinal worms and also relieving for fever and } \\
\text { bodyache (Jhapa: [66]). }\end{array}$ \\
\hline 521 & Thamnocalamus sp. & Herb & Poaceae & Bans (Np) & & Shoots: Used forroofing, mats and fencing (KL Bhutan: [100]). \\
\hline 522 & $\begin{array}{l}\text { Thysanolaena maxima } \\
\text { (Roxb.) Kuntze }\end{array}$ & Herb & Poaceae & Amriso (Np) & $100-2000$ & $\begin{array}{l}\text { Roots: Extract used in boils and worms (Ilam: [73, 76, 77]; Sikkim: [79]). Paste } \\
\text { applied on boils (Darjeeling: [52]). Decoction of young roots taken in case of } \\
\text { bronchial problem (Darjeeling: [49]). Leaves: Used as abortifacient (Panchthar: } \\
\text { [67]). Flowers: Poultic of young flowers used in rheumatic pain and skin } \\
\text { swelling (Darjeeling: [49]). Inflorescence: Used to make broom (KL Bhutan: } \\
\text { [100]). }\end{array}$ \\
\hline 523 & $\begin{array}{l}\text { Polygala arillata Buch.-Ham. } \\
\text { ex D. Don }\end{array}$ & Shrub & Polygalaceae & $\begin{array}{l}\text { Yellow milkwort, Red eye (Eg); Cleem- } \\
\text { soon-creem, Karima, Marcha, } \\
\text { Michepnor-kung }(\mathrm{Np})\end{array}$ & $600-1800$ & $\begin{array}{l}\text { Roots: Juice taken for its laxatic and emetic actions (Sikkim: [85]). Plant: Used } \\
\text { to make marcha (Darjeeling: [80]). }\end{array}$ \\
\hline 524 & Bistorta vivipara (L.) Gray & Herb & Polygonaceae & $\begin{array}{l}\text { Pantsa Ramba, Rambu (Sh); Pangram, } \\
\text { Rmbu Godpa (Tb) }\end{array}$ & $3300-5000$ & Plant: Used as fodder (Taplejung: [87]). \\
\hline 525 & $\begin{array}{l}\text { Fagopyrum esculentum } \\
\text { Moench }\end{array}$ & Herb & Polygonaceae & Phapar (Li) & $1800-4100$ & Leaves: Juice taken orally during stomachache and gastritis (Sikkim: [79]). \\
\hline 526 & Oxyria digyna (L.) Hill & Herb & Polygonaceae & Mountain Sorrel (Eg); Boke (Np) & $2400-5000$ & Plant: Used as fodder (Taplejung: [87]). \\
\hline 527 & $\begin{array}{l}\text { Persicaria barbata (L.) H. } \\
\text { Hara }\end{array}$ & Herb & Polygonaceae & Bish (Np); Bareputuli (Me) & $200-2500$ & $\begin{array}{l}\text { Leaves: Paste with cloves of Allium sativum applied on ringworm after slightly } \\
\text { scouring the wound by warm salt-water (Jhapa: [68]). }\end{array}$ \\
\hline
\end{tabular}
Hara

Polygonaceae

scouring the wound by warm salt-water (Jhapa: [68]). 
Table 1 NTFPs used by the local people of the Kangchenjunga Landscape, Eastern Himalaya (Continued)

528 Polygonum coriaceumSam. Herb

529 Polygonum molle D. Don. Herb

530 Polygonum runcinatum Buch.Ham. ex D. Don

531 Polygonum sp.

532 Polygonum verticillatum Biroli ex Colla

533 Polygonum viviparum L.

534 Rheum acuminatum Hook. Herb f. \& Thoms. ex Hook

535 *Rheum australe D. Don

536 Rheum nobile Hook. f. \& Thoms.

537 Rumex nepalensis Spreng

538 Portulaca oleracea L. Herb

539 Androsace hookeriana Klatt Herb
Polygonaceae

Halhale (Np)

$1200-4200$

\section{Polygonaceae}

Polygonaceae

Polygonacea

Polygonaceae

Polygonaceae

Polygonaceae

Polygonaceae

Padamchal (Np); Bokya (Np-Dl); Khokkim (Np-Tp); Chhurta, Khokkim Kyojung (Sh); Chum Tsa (Tb)

Himalayan Rhubarb (Eg); Chuk, Chulth Amilo, Mire Chuk, Padamchal (Np): Khokkim (Np-Tp); Chhulama

Chhoyoma, Churcha (Sh): Amlaparni, Pitamulika (Sn); Chhucha (WI)

Padamchal (Np): Kenjo, Chhulama (Sh, Wl); Chuka (Dz)

$3600-5000$

Portulacaceae

Kulfa Saag (Np)

Primulaceae
Roots: Used as medicine (KL Bhutan: [71])

Plant: Juice taken to treat diarrhea (llam: [76, 77]; Sikkim: [85]). Used as astringent (Sikkim: Database). Young shoots: Used for curry (llam: [94]) and pickle (llam: [73, 94]; Sikkim: Database). Useful in diarrhoea (llam: [73]). Leaves: Used as fodder (Taplejung: [81, 87]).

Whole plant: Used to make vegetable (llam: [94].

Roots: Used as medicine (KL Bhutan: [71]).

Roots: Used as medicine (KL Bhutan: [71]).

Roots: Juice boiled with water and given in fever (Sikkim: [11], Database), recommended for jaundice (Sikkim: Database) and stomach trouble (Sikkim: [11], Database). Leaves: Paste applied on insect bite (Darjeeling: [74]).

Roots: Used as medicine (KL Bhutan: [71]). Herbal tea prepared from rootstock used to treat body pain; paste applied on forehead during severe headache (Sikkim: [79]). Petioles: Eaten raw (Taplejung: [87]) and also as appetize (Sikkim: [79]). Leaves: Juice from the shoot portion taken in dysentery and intestinal problems (Sikkim: [79]).

Roots: Dried and used as tea (Darjeeling: [80]). Taken in constipation, skin eruption, and liver complaints (Sikkim: [75]). Decoction used as tea during internal injuries, body pain, and for blood purification. Rootstocks also yields dye for coloring wool (Taplejung: [81, 87]; KL Nepal: [83]; Sikkim: Database). Rhizome powder taken in diarrhoea, dyspepsia, constipation and ulcer (llam [76]). Petiole: Young petioles and leaves used to cure cuts and wounds, sprains and bodyache (Sikkim: [90]). Fresh petioles eaten raw (Taplejung: [81, 87]; KL Nepal: [83]; Sikkim: Database).

Roots: Used as medicine (KL Bhutan: [71]).Effective in ulcer, bronchitis, and

fever (Sikkim: [75]). Boiled and paste with flour and Tibetan salt applied on the fractured area. Boiled with rhizome of Begonia and paste applied externally on the fractured bone (Taplejung: $[81,87])$. Decoction or infusion aken to treat rheumatism, arthritis, heart complaints and as tonic after delivery (Darjeeling: [49]). Used as anti emetic, diuretic, laxative, heating potency and for swelling (Darjeeling: [98]). Plant: Consumed as vegetable or pickle (Darjeeling: [80, 98]).

Roots: Extract used orally in hepatitis and loss of hair (Darjeeling: [80]; Sikkim: [11, 89], Database) and as dyes (Sikkim: Database). Infusion taken to treat ulcer, cuts, and sprains (Darjeeling: [91]; llam: [73, 76, 77]). Shoots: Used in skin diseases (Sikkim: [75]). Leaves and shoots: Juice applied on woundto hea (Darjeeling: [86]; Sikkim: [1 1]). Leaves: Cooked and taken as vegetable (llam: [94]). Leaf and root paste taken in stomachache and applied on wound (KL Nepal: [53]).

Young plants: Used as vegetable (Jhapa: [92])

Roots: Used as medicine (KL Bhutan: [71]). 
Table 1 NTFPs used by the local people of the Kangchenjunga Landscape, Eastern Himalaya (Continued)

540 Primula sikkimensis Hook. Herb

541 Helicia nilagirica Bedd. Tree

542 Aconitum ferox Wall. exSer. Herb

543 Aconitum heterophyllum Herb Wall. ex Royle

544 Aconitum lethale Griff. Herb

545 Aconitum naviculare (Brühl) Herb Stapf

546 Aconitum orochryseum Herb Stapf

547 Aconitum palmatum D.Don Herb

548 *Aconitum spicatum (Bruhl) Herb Stapf

549 Anemone rivularis Buch.- Herb Ham. ex DC.

550 Clematis acuminata DC. Climber

551 Clematis buchananiana DC. Climber

552 Clematis montana Buch.- Climber Ham. ex DC.

553 Delphinium brunonianum Herb Royle

554 Delphinium cashmerianum Herb Royle

555 Thalictrum chelidonii DC. Herb
Primulaceae

Proteaceae

Ranunculaceae

ndian Aconite (Eg): Bikh, Bish Atisingua bish, Seto bikhma (Np)

2100-3800

Ranunculaceae Bikh, Atish (Np)

Ranunculaceae

Manchhen (Sh), Tsenduk (Dz)

Ranunculaceae

Ranunculaceae

Ranunculaceae

Ranunculaceae

Ranunculaceae

Ranunculaceae

Ranunculaceae

Ranunculaceae

Ranunculaceae

Ranunculaceae

Ranunculaceae

Bong-nga (Sh)

Kuphora Bikhma (Li); Lungezee nyin (Lp); Bikhma, Nirmasi (Np); Bongmar, Bongser, Pomar (Sh); Bhungna (WI)

$3500-4500$

Seto Bikhuma (Np)

$1800-4200$

Pinasay Lahara (Np)

Tinpate Lahar, Pinasay Lahara (Np); Maha Gagri (Np-Tp); Bhwaresang, Thaknangjwa (Ri); Pipipma (Sh); Khondro Langdu (Tm); Imong-Karpi (Tb)

Kaneshi Lahara (Np); Lanitokaru (Sh)

\section{Whole plant: Used as medicine (KL Bhutan: [71]).}

Leaves: Use to extract dye (KL Bhutan: [70]).

Plant used as expectorant, febrifuge and in diabetes (Sikkim: [89]). Rhizomes: Used in malaria and other fevers, abdominal pains, diarrhoea and indigestion particularly for children (Darjeeling: [69]). Given in cough, skin disease (Sikkim: [11, 85], Database), asthma, leprosy and snakebite (Sikkim: Database). Decoction taken orally to treat abdominal disorders (Darjeeling and Sikkim: [69]; Sikkim: [85]; KL Nepal: [53]). Dried rhizome juice taken as antipyretic agent (Darjeeling: [78]; Sikkim: [11, 90]). Dried rhizome chewed in food poisoning, cold and cough (Sikkim: [79]). Chewed in headache, powder taken orally to cure nervousness and heart problems (llam: [76]; KL Nepal: [53]).

3200-3700 Rhizomes: Used as bitter tonic and antidote for snakebite (Darjeeling: [69]); taken orally against food poisoning (Darjeeling: [52]). Used as anti-fertility agent, tonic, stomachic, anti-periodic and given in hysteria, piles and throat diseases (Sikkim: Database). Dried rhizomes taken to cure body ache (Sikkim: [11]; Darjeeling: [52]), fever, cold, cough and nose discharge (Sikkim: [11, 91]).

Roots: Used as medicine (KL Bhutan: [71]).

Rhizomes: Used as medicine (KL Bhutan: [71]).

Rhizomes: Used as medicine (KL Bhutan: [71]).

Roots: Powder used to treat fever (Taplejung: [81, 88]; Sikkim: [75]), headache, stomachache (Taplejung: [81, 82, 87, 88]) and rheumatism (Sikkim: [75]).

Rhizomes: Used for cholera, rheumatism (KL Nepal: [83]), and also to treat rabies and stomachache (KL Nepal: [53]). Paste given in diarrhea (llam: [73]). Buti (Amulet) prepared with tuber and tied on the body of a baby to keep far from bad spirit (Taplejung: [81])

Fruits: Used as medicine (KL Bhutan: [71]).

Roots: Given to treat sinusitis (Sikkim: [75]).

Roots: Juice or powder used in headache, sinusitis (Taplejung: [87]; Sikkim: [11, 75, 89], Database), half headache and to make fermenting agent (Taplejung: [87]). Fresh roots crushed and used to release effluvium through nose to cure sinusitis and nose blocks (Darjeeling: [52, 74, 80]).

Roots: Taken to cure sinusitis (Sikkim: [75]). Seeds/flowers: Used as medicine (KL Bhutan: [71]).

Whole plant: Used as medicine (KL Bhutan: [71]) and incense (KL Bhutan: [70]).

Whole plant: Used as medicine (KL Bhutan: [71]).

Roots: Used as tonic and purgative (Sikkim: [75]). 
Table 1 NTFPs used by the local people of the Kangchenjunga Landscape, Eastern Himalaya (Continued)

\begin{tabular}{|c|c|c|c|c|c|c|}
\hline 556 & Thalictrum foliolosum DC. & Herb & Ranunculaceae & Dampatey $(\mathrm{Np})$ & $1300-3400$ & $\begin{array}{l}\text { Roots: Used as medicine (KL Bhutan: [71]). Used as tonic and purgative } \\
\text { (Sikkim: [75]). Decoction taken in fever (Darjeeling: [78]). }\end{array}$ \\
\hline 557 & Thalictrum reniforme Wall. & Herb & Ranunculaceae & & $2800-3300$ & $\begin{array}{l}\text { Roots: Used to treat jaundice (Panchthar: [67]). Paste applied to cheek boils } \\
\text { (Sikkim: [11], Database). Decoction used as gargle in case of bad breath and } \\
\text { kills worms in stomaching on drinking (Sikkim: [1 1]). }\end{array}$ \\
\hline 558 & Gouania leptostachya DC. & Climber & Rhamnaceae & & & Leaves: Paste applied to cure sores and inflammation (Sikkim: [11]). \\
\hline 559 & Hovenia dulcis Thunberg & Tree & Rhamnaceae & Coral tree (Eg); Bangikath (Np) & $200-1400$ & $\begin{array}{l}\text { Seed: Powder taken to get relief from excessive drinking of alcohol (Sikkim: } \\
\text { [85]). }\end{array}$ \\
\hline 560 & $\begin{array}{l}\text { Rhamnus napalensis (Wall.) } \\
\text { M. A. Lawson }\end{array}$ & Shrub & Rhamnaceae & Archal (Np) & & $\begin{array}{l}\text { Roots: Used as purgative (Sikkim: [75]). Roots and leaves: Applied on } \\
\text { fractured bone (Sikkim: [75]). }\end{array}$ \\
\hline 561 & Zizyphus mauritiana Lam. & Tree & Rhamnaceae & Janum (S); Bayer (Np) & $200-1200$ & $\begin{array}{l}\text { Roots, seeds and bark: Useful in measles and pneumonia (Panchthar: [67]). } \\
\text { Bark: Extract used in diarrhea; powder used for septic wounds (Jhapa: [66]). } \\
\text { Fruit: Eaten raw (Jhapa: [92]). }\end{array}$ \\
\hline 562 & Zizyphus rugosa Lam. & Shrub & Rhamnaceae & Sekra (S) & $150-800$ & Fruit: Eaten raw (Jhapa: [92]). \\
\hline 563 & Zizyphus spp. & Shrub & Rhamnaceae & Khankarisey, Khanglchalingsay (Sh) & & Fruit: Edible (KL Bhutan: [70]). \\
\hline 564 & $\begin{array}{l}\text { Chaenomeles speciosa } \\
\text { (Sweet) Nakai }\end{array}$ & Shrub & Rosaceae & & & Flowers and fruit: Use to extract dye (KL Bhutan: [70]). \\
\hline 565 & $\begin{array}{l}\text { Cotoneaster microphyllus } \\
\text { Wall. ex Lindl. }\end{array}$ & Shrub & Rosaceae & & $2000-5000$ & $\begin{array}{l}\text { Roots: Used as astringent (Sikkim: [75]). Fruit: Used as medicine (KL Bhutan: } \\
\text { [71]). }\end{array}$ \\
\hline 566 & $\begin{array}{l}\text { Docynia indica (Wall.) } \\
\text { Decne. }\end{array}$ & Tree & Rosaceae & $\begin{array}{l}\text { Mayel (Np); Thungchurpu (Sh); Tong } \\
\text { (Dz) }\end{array}$ & $2000-3000$ & Fruit: Used to make jam and pickles (Ilam: [94]). Edible (KL Bhutan: [70]). \\
\hline 567 & $\begin{array}{l}\text { Duchesnea indica (Andrews) } \\
\text { Focke }\end{array}$ & Herb & Rosaceae & Bhui Aiselu (Li) & $1000-25000$ & Leaves: Paste applied on cut and wounds (Darjeeling: [74]). \\
\hline 568 & $\begin{array}{l}\text { Fragaria nubicola Lindl. ex } \\
\text { Lacaita }\end{array}$ & Herb & Rosaceae & Bhui Aiselu (Li) & $1600-4000$ & $\begin{array}{l}\text { Roots: Paste applied externally to control bleeding; chewed in cough and cold } \\
\text { (Sikkim: [79]). Juice taken in cough and cold, toothache, and high altitude } \\
\text { sickness (Darjeeling: [74]). Fruit: Ripen fruits cosumed (KL Bhutan: [70]; llam: } \\
\text { [94]). Leaves and fruit: Pounded and administered with lukewarm water to } \\
\text { cattle to treat diarrhea and dysentery, also used as diuretic for cattle (Sikkim: } \\
\text { [84]). }\end{array}$ \\
\hline 569 & Potentilla fructicosa $\mathrm{L}$. & Herb & Rosaceae & $\begin{array}{l}\text { Teba, Pangsermendo (Gr-Mn); Pema, } \\
\text { Pama, Pang Ser Metog (Km); Bhairung } \\
\text { Pate, Jhwani (Np) }\end{array}$ & $2700-4600$ & $\begin{array}{l}\text { Roots: Used for breast disease, stomach and lung disorders, and indigestion; } \\
\text { also used as incense (KL Nepal: [83]). Stem: Used as brooms (Taplejung: [87]). } \\
\text { Leaves and flowers: Used in making tea (Taplejung: [87]). }\end{array}$ \\
\hline 570 & $\begin{array}{l}\text { Potentilla fulgens Wall. ex } \\
\text { Hook. }\end{array}$ & Herb & Rosaceae & Bajradanti (Np) & $1600-4800$ & $\begin{array}{l}\text { Plant: Juice taken to treat stomach trouble, cough, and cold. Decoction given } \\
\text { to cattle to regulate fertility (Sikkim: [97]). Roots: Powder used to cure } \\
\text { toothache and diarrhea (Sikkim: [75]) and pyorrhea. Juice used in throat and } \\
\text { tooth infection, peptic ulcer, cough and cold (Ilam: [73, 76]). Root stock used to } \\
\text { treat gastric troubles (Panchthar: [67]). }\end{array}$ \\
\hline 571 & Prinsepia utilis Royle & Shrub & Rosaceae & Phekray (Np) & $1500-2900$ & Seed: Oil consumed (llam: [94]). \\
\hline 572 & Prunus cerasoides D. Don & Tree & Rosaceae & Payun, Aaru Patay (Np) & $1300-2400$ & $\begin{array}{l}\text { Bark: Crushed and applied on the injuries of cattle (Sikkim: [11, 97]). Decoction } \\
\text { taken to treat fever (Darjeeling: [78]). Bark and stems: Applied on fractured } \\
\text { bone and toothache (Sikkim: [75]). Fruit: Edible (llam: [94]; Sikkim: [11, 95]). }\end{array}$ \\
\hline
\end{tabular}


Table 1 NTFPs used by the local people of the Kangchenjunga Landscape, Eastern Himalaya (Continued)

\begin{tabular}{|c|c|c|c|c|c|}
\hline 573 & $\begin{array}{l}\text { Pyrus pashia Buch.-Ham. ex } \\
\text { D. Don }\end{array}$ & Tree & Rosaceae & Mayal (Np) & $750-2600$ \\
\hline 574 & Rosa macrophylla Lindl. & Shrub & Rosaceae & Himalayan Rose (Eg); Jangali gulaf (Np) & $1400-3300$ \\
\hline 575 & Rosa sericea Lindl. & Herb & Rosaceae & $\begin{array}{l}\text { Sewa (Gr-Mn); Sewa Metog (Km); } \\
\text { Sebimendo (Sh); Segue-Karpo, Seroga, } \\
\text { Sewa (Tb); Setokpa (WI) }\end{array}$ & $2200-4600$ \\
\hline 576 & Rubus ellipticus Sm. & Shrub & Rosaceae & $\begin{array}{l}\text { Nepali Raspberry (Eg); Tinsek (Li); } \\
\text { Kysyim (Lp); Aiselu, Aeiselu (Np); Ghees, } \\
\text { Tudum (Ri); Chhilum, Nyaningma (Sh); } \\
\text { Polang (Tm); Kandakari, Gah-Trah (Tb); } \\
\text { Chhilum (WI) }\end{array}$ & $1700-2600$ \\
\hline 577 & Rubus lineatus Reinw. & Shrub & Rosaceae & Suvuk (L) & \\
\hline 578 & Rubus rugosus Sm. & Shrub & Rosaceae & Jogi Ainselu (Np) & 1500 \\
\hline 579 & $\begin{array}{l}\text { Anthocephalus chinensis } \\
\text { (Lam.) A. Rich. ex Walp. }\end{array}$ & Tree & Rubiaceae & Kadam (S and Np) & $290-800$ \\
\hline 580 & $\begin{array}{l}\text { Anthogonium gracile Wall. } \\
\text { ex Lindl. }\end{array}$ & Herb & Rubiaceae & Bhui Sunakhari (Np) & $1200-2300$ \\
\hline 581 & Cinchona officinalis $L$. & Shrub & Rubiaceae & Sinchona (Np) & \\
\hline 582 & Galium aparine L. & Herb & Rubiaceae & Zangtsi-rokpu (Sh) & \\
\hline 583 & $\begin{array}{l}\text { Haldina cordifolia } \\
\text { (Roxburgh) Ridsdale }\end{array}$ & Tree & Rubiaceae & Pahenley (Np); Sinjo (S) & \\
\hline 584 & $\begin{array}{l}\text { Hedyotis corymbosa (L.) } \\
\text { Lam. }\end{array}$ & Herb & Rubiaceae & Piriengo (Np) & $200-2400$ \\
\hline 585 & Hedyotis scandens Roxb. & Herb & Rubiaceae & Bokre Lahara (Np); Kalhya (L) & $400-1800$ \\
\hline 586 & Houstonia spp. & Herb & Rubiaceae & Nombareng (Sh) & \\
\hline 587 & $\begin{array}{l}\text { Meyna pubescens (Kurz) } \\
\text { Robyns }\end{array}$ & Shrub & Rubiaceae & & 200 \\
\hline 588 & Mussaenda frondosa $\mathrm{L}$. & Shrub & Rubiaceae & Dhotisara (Np) & \\
\hline
\end{tabular}

Fruit and bark: Taken to cure menstrual disorders (llam: [73]). Fruit: Extract taken to cure dysentery (Darjeeling: [80]).

Fruit: Used as medicine (KL Bhutan: [71]). Used for fever, diarrhea, and bile disorders; edible and also used for making local wine (KL Nepal: [83]).

Bark: Used in cases of poisoning and lymph fluid disorders (KL Nepal: [83]). Flower and ripe fruit: Used in liver, bile, wind and lung diseases and menstrual disorders. Ripe fruit: Eaten raw (Taplejung: [81]). Believed to have curative effects in headache, liver complaints, and constipation (Sikkim: [75, 79]). Leaves: Decoction used to wash wound (Sikkim: [79]). Flowers: Used as medicine (KL Bhutan: [71]).

Roots and bark: Useful in gastric problem and diarrhea (Darjeeling: [49, 74]; Panchthar: [67]). Roots: Juice given in fever (Sikkim: [75]). Paste applied on forehead during severe headache (Sikkim: [11]) and applied to treat wound (Sikkim: [11]). Root used for abortion (Darjeeling: [52]). Roots and young shoots: Used for colic pain (Sikkim: [11]). Young shoots: Chewed to stop sudden stomach pain (Darjeeling: [51, 91]; Sikkim: [11]), mouth allergy and paste of tender shoots applied as antidote to snake bite (Taplejung: [87]). Tender leaves and/or young stem shoots pounded and administered to livestock to treat cold and fever (Sikkim: [84]]. Bark: Crushed and applied on injuries of cattle (Sikkim: [97]). Fruit: Used as spices (KL Bhutan: [70]). Edible (Darjeeling: [80]; llam: [94]; Sikkim: [95]). Juice used to cure fever and cough; ripe fruits used to make local wine (Taplejung: [88]) and in dysentery and wounds (llam: [73]).

Roots: Taken in food poisoining (Sikkim: [75]).

Root bark: Used as anthelmintic drug (Panchthar: [67]).

Fruit: Ripe pseudocarps edible (Jhapa: [68]).Consumed during stomachache (Sikkim: [79]). Seeds: Roasted and oil consumed (llam: [94]).

Rhizomes and pseudobulbs: Paste applied externally to treat bone fracture and dislocation; pseudobulbs crushed to make paste and applied externally to cure boils (Darjeeling: [52]).

Bark: Used as remedy for malaria (Sikkim: Database)

Whole plant: Used as medicine (KL Bhutan: [71]).

Bark: Decoction used for antipyretic actions (Darjeeling: [78]). Young shoots: Decoction applied in eye infections (Jhapa: [66]).

Plant: Decoction taken orally to prevent gastric irritability and also act as an anthelmintic (Darjeeling: [74]; Sikkim: [85]).

Whole plant: and roots are useful in eye diseases, sprains and boils (Sikkim: [75]). Roots: Useful in eye diseases, sprains, and boils (Sikkim: [75]).

Leaves: Used as spices (KL Bhutan: [70]).

Young leaves: Used as vegetable (Jhapa: [92]). Fruit: Edible (Jhapa: [92]).

Plant: Decoction given to treat fever, cough, and asthma (Sikkim: [1 1]), Leaves: Extract given in fever (Darjeeling: [78]). Roots: Juice given in jaundice
(Darjeeling: [74]). 
Table 1 NTFPs used by the local people of the Kangchenjunga Landscape, Eastern Himalaya (Continued)

\begin{tabular}{|c|c|c|c|c|c|c|}
\hline 589 & $\begin{array}{l}\text { Mussaenda macrophylla } \\
\text { Wall. }\end{array}$ & Herb & Rubiaceae & Tungbub (L) & & $\begin{array}{l}\text { Roots: Taken in jaundice (Sikkim: [75]). Juice taken in acidity, diabetes and } \\
\text { fever (Ilam: [76]). }\end{array}$ \\
\hline 590 & Mussaenda treutleri Stapf & Shrub & Rubiaceae & Tungbub (L) & & Roots: Given in jaundice (Sikkim: [75]). \\
\hline 591 & Paederia foetida $\mathrm{L}$. & Climber & Rubiaceae & Barilahara (Np) & $300-1800$ & Leaves: Decoction used as medicine (Darjeeling: [78]). \\
\hline 592 & $\begin{array}{l}\text { Paederia scandens (Lour.) } \\
\text { Merr. }\end{array}$ & Shrub & Rubiaceae & Pate biree (Np) & 1400 & $\begin{array}{l}\text { Stem: Swollen part of the stem used to treat rheumatism (Panchthar: [67]). } \\
\text { Fruit: Dried and paste applied around tooth to cure toothache and prevent } \\
\text { tooth decay (Sikkim: [11]). }\end{array}$ \\
\hline 593 & Pavetta indica $\mathrm{L}$. & Tree & Rubiaceae & $\begin{array}{l}\text { White Pavetta (Eg); Kangyphul, Takali, } \\
\text { Sundok (Np) }\end{array}$ & & Roots: Juice used for purgative action (Sikkim: [85]). \\
\hline 594 & Randia sp. & Tree & Rubiaceae & Nertingaey (Sh) & & Fruit: Edible (KL Bhutan: [70]). \\
\hline 595 & Rubia cordifolia L. & Herb & Rubiaceae & Majito & & $\begin{array}{l}\text { Roots and fruit: Taken in dysentery, uterian pains, and voice complexion } \\
\text { (Sikkim: Database). Stem: Crushed, mixed with rice wash and given to cattle in } \\
\text { post natal problem of cattle (Sikkim: [97]). Roots: Used as medicine (KL Bhutan: } \\
\text { [71]). Decoction of dried roots taken to treat fever (Darjeeling: [78]). Decoction } \\
\text { used in urinary infection; paste applied on skin diseases (Darjeeling: [91]; } \\
\text { Sikkim: [11]). Used as dye (Darjeeling: [72]; Sikkim: [11]). Paste applied on } \\
\text { forehead to cure headache (Darjeeling: [52]). Paste also used as ointment to } \\
\text { treat skin infection; decoction administered to cattle after delivery for quick } \\
\text { recovery (Sikkim: [84]). }\end{array}$ \\
\hline 596 & $\begin{array}{l}\text { *Rubia manjith Roxb. ex } \\
\text { Fleming }\end{array}$ & Climber & Rubiaceae & Majitho (Np) & $1200-2100$ & $\begin{array}{l}\text { Plant: Used as dye (Darjeeling: [80]; Sikkim: Database). Decoction used to treat } \\
\text { snake bite, desentery, leprosy, skin diseases (Darjeeling: [74]; Ilam: [73, 76]), } \\
\text { diabetes, and arthritis (llam: [73, 76]). Roots: Used as alterative, astringent, and } \\
\text { tonic. Used to obtain dye (KL Nepal: [83]). Used as tonic, alternative, astringent } \\
\text { (Sikkim: Database). Stem: Used in scorpion bite (Sikkim: Database). Roots and } \\
\text { fruit: Given to treat menstrual disorders (Darjeeling: [49]; Sikkim: [75]). }\end{array}$ \\
\hline 597 & Rubia wallichiana Decne. & Climber & Rubiaceae & Vyem (L) & $300-2600$ & Young shoots: Taken in jaundice and paralysis (Sikkim: [75]). \\
\hline 598 & $\begin{array}{l}\text { Spermadictyon suaveolens } \\
\text { Roxb. }\end{array}$ & Shrub & Rubiaceae & Ban Champ (Np) & & Roots: Paste applied externally to relieve from joint pain (Sikkim: [11]). \\
\hline 599 & $\begin{array}{l}\text { Tamilnadia uliginosa (Retz.) } \\
\text { Tirveng. \& Sastre }\end{array}$ & Tree & Rubiaceae & Pendra (S); Pidar (Np) & 500 & $\begin{array}{l}\text { Bark: Decoction taken orally to treat diarrhea and dysentery (Sikkim: [85]). } \\
\text { Fruit: Unripe fruits eaten as vegetable (Jhapa: [92]). }\end{array}$ \\
\hline 600 & Uncaria sessilifructus Roxb. & Climber & Rubiaceae & & 200-900 & Root: Used to treat bone fracture and crack (Panchthar: [67]). \\
\hline 601 & $\begin{array}{l}\text { Uncari arhynchophylla } \\
\text { (Miq.) Miq. ex Havil. }\end{array}$ & Climber & Rubiaceae & & & Whole plant: Used as medicine (KL Bhutan: [71]). \\
\hline 602 & Aegle marmelos (L.) Correa & Tree & Rutaceae & Sinjo (S); Bel (Np) & $600-1100$ & $\begin{array}{l}\text { Fruit: Used as medicine (KL Bhutan: [71]). Used in constipation, diarrhea and } \\
\text { dysentery (Jhapa: [66]; Panchthar: [67]; Darjeeling: [86]; Sikkim: [79, 91]). Pulp } \\
\text { edible (KL Bhutan: [70]; Jhapa: [92]). Seeds: Extract taken orally in ulcer (KL } \\
\text { Nepal: [53]). }\end{array}$ \\
\hline 603 & $\begin{array}{l}\text { Boenninghausenia albiflora } \\
\text { (Hook.) Rchb. ex Meisn. }\end{array}$ & Herb & Rutaceae & Likhijari (Np) & $600-3300$ & $\begin{array}{l}\text { Leaves: Extract applied on the body of cattle to kill lice and flea (Sikkim: [75, } \\
\text { 97]). }\end{array}$ \\
\hline 604 & Citrus medica L. & Tree & Rutaceae & Bimbira (Li) & $700-1200$ & $\begin{array}{l}\text { Fruit: Eaten raw in indigestion, vomiting, jaundice and typhoid. Dried skin } \\
\text { powder administered in dysentery (Sikkim: [79]). }\end{array}$ \\
\hline
\end{tabular}


Table 1 NTFPs used by the local people of the Kangchenjunga Landscape, Eastern Himalaya (Continued)

\begin{tabular}{|c|c|c|c|c|c|}
\hline 605 & Citrus reticulata Blanco & Tree & Rutaceae & Suntala (Li) & $600-1800$ \\
\hline 606 & Clausena excavata Burm. f. & Shrub & Rutaceae & & $200-500$ \\
\hline 607 & $\begin{array}{l}\text { Euodia fraxinifolia (D.Don) } \\
\text { Hook. f. }\end{array}$ & Tree & Rutaceae & Khanakpa (Np) & $1000-2400$ \\
\hline 608 & Evodia fraxinifolia Hook. f. & Tree & Rutaceae & Khanakpa, Kanu (Np) & $1200-2100$ \\
\hline
\end{tabular}

Fruit: Skin dried and paste applied on the face to soften (Sikkim: [79]).

Leaves: Used to remove flies from wounds, sores, and cuts (Jhapa: [66]).

Bark: Juice given in dysentery and menstrual disorder (llam: [73]).

Roots: Powder taken to treat dysentery (Darjeeling: [80]; Sikkim: [85]). Bark: Decoction given in fever (Darjeeling: [78]). Fruit: Used as antipyretic and in treatment of typhoid; eaten as vegetable (Darjeeling: [80]; Sikkim: [89]). Seeds: Used to make chutney and taken with food to improve appetite (Darjeeling: [91]; Sikkim: [79]).

609 Murraya koenigii (L.) Spreng. Tree Rutaceae

610 Tetradium fraxinifolium Tree (Hooker) T. G. Hartley

611 Zanthoxylum acanthopodium DC

Shrub Rutaceae

Rutaceae

Khanakpa (Np); Kanu (L)

700-3000

Boke timur (Np); Gee (Sh), Thingne (Dz) 1600-2800

613 Zanthoxylum budrunga Shrub Wall.

614 Zanthoxylum oxyphyllum Shrub Edgew.

615 Salix babylonica L. Tree

616 Salix calyculata Hook. f. ex Shrub Andersson

617 Osyris wightiana Wall. Ex Shrub Wight

618 Pyrularia edulis (Wall.) A. DC. Tree

619 Cardiospermum Climber halicacabum L.

Sapindaceae

Sapindaceae

Rutaceae

Timur (Np)

$1100-2500$

Nepalese Pepper (Eg): Ban Timur, Lekh ～2100-2800 Timur (Np)

\section{Bains (Np)}

Leng Junye, Langma Parma (Km): 3600-4500 Lansip (Sh); Langma Chhung (Tb); Langma (WI)

Nundhiki (Np)

Amphi (Np)

Fuka fucha (S)

620 *Sapindus mukorossi Gaertn. Tre

Leaves: Used as spices (KL Bhutan: [70]).

Whole plant: Taken as antipyretic and diuretic (Sikkim: [75]). Fruit: Taken as antipyretic and diuretic (Sikkim: [75]).

Leaves and seeds: Used to cure toothache and worms (Darjeeling: [91]; Ilam: [73]). Branchlets: Used as toothbrush to relieve toothache (Sikkim: [11]). Fruit: Taken in indigestion (Darjeeling: [80]; Sikkim: [74, 75, 79]), used to treat ear diseases, headache, and asthma (Darjeeling: [80]; Sikkim: [89]). Crushed an rubbed on the leg and foot as antileech repellent agent (Darjeeling: [91]; Sikkim: [11]).Used as medicine (KL Bhutan: [71]) and as spices (KL Bhutan: [70]).

612 *Zanthoxylum armatum DC Shrub

Fruit, roots and leaves: Decoction used to treat toothache, fever, cough

reumatism, and asthma (Darjeeling: [78]; KL Nepal: [83]; Panchthar: [67]; Ilam: [73, 76, 77]). Fruit: Used as spice (KL Nepal: [83]; Panchthar: [67]; llam: [73]),

Fruit: Used as spices (KL Bhutan: [70]).
$1400-3600$

$1100-2600$

1600-1800

900-1500

Fruits: Pickled and eaten; paste of immature fruit kept between teeth to relieve from toothache (KL Nepal: [83]). Flowers and fruit: Extract given to treat pain, tumor, fever, cholera and snake bite (llam: [76]).

Leaves and stem bark: Infusion taken in fever (Darjeeling: [78]).

Plant: Used in death ritual. Leaves and flowers: Used as incense (Taplejung: $[81,88])$

Root bark: Used to treat body pain and fractured bone (Panchthar: [67]).

Fruit: Edible; kernel possess wax which is used for lighting (Sikkim: [89]).

Plant: Powder mixed with mustard or coconut oil applied to cure sores and wounds (Jhapa: [66])

1000-1200 Fruit: Juice used to cure burnt part of the body; also used for epilepsy (Sikkim Database). Extract applied on head to remove dandruff and lice (Darjeeling and Sikkim: [69]; Sikkim: [11]). Used to extract oil (llam: [94]). Fruit and root bark: Used as tonic, anthelmintic, purgative, in asthma and piles (Darjeeling and Sikkim: [69]). 
Table 1 NTFPs used by the local people of the Kangchenjunga Landscape, Eastern Himalaya (Continued)

Scheichera oleosa (Lour.) Tree

200-300

622 Aesandra butyracea (Roxb.) Tree

Sapotaceae

Chiuri (Np)

Baehni

623 Diploknem abutyracea Tree (Roxb.) H.J.Lam

624 Madhuca longifolia (Roxb.) Tree Macbride

625 Houttuynia cordata Thunb. Herb

626 Astilbe rivularis Buch.Ham. Herb ex D. Don

Sapotaceae

Sapotaceae

Saururaceae

Saxifragaceae

627 *Bergenia ciliata (Haw.) Sternb.

Rockfoil (Eg); Pakhanved (Np); Sallipat, Salpari, Simtadi (Np-DI); Sediwakthosida (Ri); Pashanaveda (Sn); Hyoma, Kopsokpa, Silviro (Sh); Bhramhendo (Tm)

Tangphung, Tangphung Supari, Buriokahti, Budo Okhati, Thulo Ausad (Np); Thappasid (Ri); Tongsergugay (Sh)

150-300

$1300-2500$

2000-3600

628 Bergenia purpurascens (Hook. f. \& Thomson) Engl.

Herb

Saxifragaceae

Pakhanved (Np); Chhurcha, Kopsyokpa

$3800-4700$ (Sh); Gadur, Ligadur (Tb)

Churtsa, Simjhar (Sh); Ya Ki Ma, Ser Ya Ki Ma (Tb)

629 Chrysosplenium carnosum

Herb

*Neopicrorhiza

scrophulariiflora (Pennell)

Herb

Hong
Saxifragaceae

Saxifragaceae
Katuko (Np-DI); Katukaa (Sn); Hogling, Hunglen (Sh, WI)
Fruits: Edible (Jhapa: [92]).

Bark and seeds: Paste applied on fractured bone and in piles (KL Nepal: [53]). Fruits: Used to extract oil (KL Bhutan: [93]). Edible ([94]; Sikkim: [11, 47, 89, 95]; KL Bhutan: [70]). Used in rheumatism (Sikkim: Database; [47]). Juice taken to soften the skin (Sikkim: [11]). Oil extract applied on body during winter (Sikkim: [79]). Leaves: Used as fodder (Sikkim: [89]).

Fruits: Edible (KL Bhutan: [70]).

Bark: Used to treat bone crack (Panchthar: [67]).

Shoots: Used as vegetable (Bhutan: [102]). Leaves: Decoction given to treat tuberculosis (Darjeeling: [74]).

Roots: Used as medicine (KL Bhutan: [71]). Taken as tonic for post natal women (Sikkim: [75]). Chewed to relief from pain (Darjeeling: [80]; Sikkim: [89]). Paste applied to treat wound and juice used in fever, dysentery, back pain waist pain, and throat pain (Taplejung: $[82,87,88])$ body ache, bleeding at pre and post pregnancy (Darjeeling: [52, 74]; Illam: [73, 76, 77]; Sikkim: [69]). Powder taken in jaundice (Sikkim: [69]). Roots and leaves: Taken in diarrhea, dysentery, and as blood purifier (Sikkim: Database). Leaves: Chewed raw in toothache (Sikkim: $[49,79])$

Roots: Used as medicine (KL Bhutan: [71]). Used as analgesic and in piles, heart diseases (Darjeeling: [80, 89]), and spleen enlargment (Darjeeling: [80]; Sikkim: [69]).Juice taken as tonic (Sikkim: [75]) and used to treat wound, boils, diarrhea (Taplejung: [87]; Sikkim: [11, 75], Database; Panchthar: [67]) sinusitis, body pain, cough and cold, eye infection, dysentery, abdominal pain, fever, and to check bleeding during menstruation (Taplejung: [87, 88]; Panchthar: [67]). Crushed and tied around the factured bone (Darjeeling: [80]), also used with root of Bergenia ciliata, Kaempferia rotunta, and Viscum articulatum for the same (Sikkim: [79]). Paste given to treat dysentery (Sikkim: [74, 91]); also applied on burns and cuts; dried rhizome used as astringent, given in ulcers and tuberculosis (Sikkim: [69]). Crushed and extract given to cattle to treat diarrhea and dysentery (Darjeeling: [86]; Sikkim: [84]).

Roots: Paste used in wound, bodyache (Taplejung: [82, 87]; Darjeeling: [80, 89]) and bone fracture (Taplejung: [82]). Decoction used in fever, diarrhea (Taplejung: [87]; Sikkim: [75]), knee ache, and loss of eye sight (Taplejung: [87]). Dried roots used as substitute of tea by high altitude dwellers to get relief from bodyache (Sikkim: [79])

Whole plant: Used as medicine (KL Bhutan: [71]). Leaves and stem: Used in common fever and typhoid fever (Taplejung: [82].

Rhizomes: Used for bile disease, eye diseases, and gastritis (KL Nepal: [83]). Decoction used to treat cold and cough, throat pain, fever, bodyache, and high blood pressure (Darjeeling: [49]; Taplejung: [81, 87, 88]). Taken in cold fever and snake bite (Darjeeling: [52]). Used as bitter tonic, laxative, stomachic, effective in dropsy and scropion stings (Sikkim: [69]). Effective in diseases of liver and spleen including jaundice and anaemia (Sikkim: [69]). 
Table 1 NTFPs used by the local people of the Kangchenjunga Landscape, Eastern Himalaya (Continued)

631 Saxifraga mucronulata Herb

Royle

632 Schiso Sm.

633 Digitalis purpurea L.

Herb

634 Lancea tibetica Hook. f. \& Thomson

635 Scoparia dulcis $\mathrm{L}$.

Herb

Scrophulariaceae

Chineebimfang (Me)

636 Smilax zeylanica L.

Climber

Smilacaceae

Solanaceae

Anisodus

638 Datura metel L

Herb

Solanaceae

Jangali phokphokey (Np) dragora officinarum $L$

642 Physalis minima L., Herb

643 Solanum aculeatissimum Herb Jaca

\section{Solanum myriacanthum} Dunal

645 Solanum nigrum L.

Solanaceae

Solanaceae

Solanaceae

Solanaceae

Solanaceae

646 Solanum sp.
Langthang, Longtankobu (WI)

Dhaturo, Dhontrey (Np)

Gofatdothrabithai (Me)

150-1500

2300-4000

300-1200

Sano bini (Np)

Khalanji (Sh.)
$100-1200$

Whole plant: Used as medicine (KL Bhutan: [71]).

Fruit: Used as tonic and given in insomnia (Sikkim: [75]).

Leaves: Used as heart tonic and cardiac stimulant (Sikkim: Database).

Whole plant: Used as medicine (KL Bhutan: [71]).

Plant: Decoction used in eye troubles (Jhapa: [66]). Leaves: Extract drunk and also applied externally on the body as a remedy for the babies weeping all days (Jhapa: [68]). Young leaves taken in diabetes (Sikkim: [75]). Roots: Decoction taken orally in diarrhea and dysentery (Jhapa: [66]).

Plant: Used in Urinary complaints and dysentery. Roots: Taken as tonic (Sikkim: Database).

Seeds: Used to treat toothache (Taplejung: [81]). Fruit: Used as medicine (KL Bhutan: [71]).

Seeds: Powder used in skin diseases in cattle (Sikkim: [97]; llam: [73, 76]), dandruff, leprosy, ulcer, and fever (llam: $[73,76])$. Burned and smoke inhaled in chronic asthmatic fits (Sikkim: [79]). Leaves: Crushed leaf applied on insect bite of cattle (Sikkim: [97]). Burnt and smoke inhaled to treat asthma (Sikkim: [11]). Warmed leaves placed on joint to reduce the pain and swelling (Sikkim: [79]). Fruit: Used as medicine (KL Bhutan: [71]). Smoked for toothache; powdered and mixed with warm mustard oil to treat earache (Jhapa: [66]). Taken in mad dog bites (Sikkim: [79])

200-2200 Seeds: Used as medicine (KL Bhutan: [71]) Used to treat scabies (Jhapa: [68]). Used as narcotic and also to treat dandruff and hairfall (Panchthar: [67]) Given orally against rabies, nervousness, nausea and hysteria (Darjeeling: [52]).

Fruit/Whole plant: Used as medicine (KL Bhutan: [71]).

Roots: Used as medicine (KL Bhutan: [71]).

Fruit: Used as tonic, diuretic, laxative, and useful in inflammations (Sikkim: Database)

Roots and fruit: Extraction taken in cold and bronchitis. Fruit: Smoked for relieving toothache (Jhapa: [66]). Seeds: Boiled and vapour taken through mouth to kill germs of teeth (Jhapa: [68]).

Seeds: Smoke directed to the infected tooth to cure toothache and tooth decay (Sikkim: [11]).

Aerial parts: Used as sedative (Darjeeling: [74]). Roots, leaves and fruit: Used in the treatment of leucoderma, dysentery, vomiting, asthma, bronchitis, fever, and urinary discharge (Sikkim: Database). Fruit: Edible (llam: [94]; Jhapa: [92]).

Fruit: Edible (KL Bhutan: [70]). 
Table 1 NTFPs used by the local people of the Kangchenjunga Landscape, Eastern Himalaya (Continued)

\begin{tabular}{|c|c|c|c|c|c|c|}
\hline$\overline{647}$ & Solanum surattense Burm. f. & Shrub & Solanaceae & Kalchudo $(\mathrm{Np})$; rangani kate $(\mathrm{S})$ & & $\begin{array}{l}\text { Roots: Grounded with Alternanthera sessilis and given in urinary troubles } \\
\text { (Jhapa: [66]). }\end{array}$ \\
\hline 648 & Solanum torvum Sw. & Shrub & Solanaceae & Henje (S); Thulo bini (Np) & $250-750$ & $\begin{array}{l}\text { Fruit: Used as vegetables and pickle (Jhapa: [92]). Fried and eaten to cure cold } \\
\text { and cough (Jhapa: [66]). Leaves: Smooked to treat toothache (Darjeeling: [74]). }\end{array}$ \\
\hline 649 & Melochia corchorifolia L. & Herb & Sterculiaceae & Thuik (S) & $200-1300$ & Young leaves: Eaten as vegetable (Jhapa: [66]). \\
\hline 650 & $\begin{array}{l}\text { Pterospermum acerifolium } \\
\text { (L.) Willd. }\end{array}$ & Tree & Sterculiaceae & Hattipaila, Numbong (Np) & $450-600$ & Flowers: Juice taken to treat peptic ulcer (Sikkim: [85]). \\
\hline 651 & Punica granatum L. & Tree & Sterculiaceae & Darim (Np) & & $\begin{array}{l}\text { Unripe fruit: Useful in vomiting, fever, heart diseases, sore throat, diarrhea and } \\
\text { dysentery (Sikkim: Database). Fruit/seeds: Used as medicine (KL Bhutan: [71]). }\end{array}$ \\
\hline 652 & $\begin{array}{l}\text { Symplocos lucida } \\
\text { (Thunberg) Siebold \& } \\
\text { Zuccarini }\end{array}$ & Tree & Symplocaceae & Kharane (Np) & $500-2600$ & $\begin{array}{l}\text { Seeds: Powder applied against spider sting (Sikkim: [75]). In the past people } \\
\text { used to extract oil for cooking from the seed (Sikkim: [89]). }\end{array}$ \\
\hline 653 & $\begin{array}{l}\text { Symplocos paniculata } \\
\text { (Thunb.) Miq. }\end{array}$ & Shrub & Symplocaceae & Pangtsi (Dz) & & $\begin{array}{l}\text { Fruit: Used as medicine (KL Bhutan: [71]), Edible (KL Bhutan: [70]). Seeds: Used } \\
\text { to extract oil (KL Bhutan: [93]). }\end{array}$ \\
\hline 654 & Symplocos sp. & Shrub & Symplocaceae & & & Leaves: Use to extract dye (KL Bhutan: [70]). \\
\hline 655 & Myricaria rosea W. W. Smith & Shrub & Tamaricaceae & $\begin{array}{l}\text { Angmeo (Gr); Thrishing (Km); Chhusin- } \\
\text { Ghumbu, Hunbu (Sh, Wi); Hunbu } \\
\text { Chungwa (Tb). }\end{array}$ & $3300-4500$ & $\begin{array}{l}\text { Whole plant: Used as medicine (KL Bhutan: }[71]) \text {.Leaves and flowers: Used } \\
\text { for incense (Taplejung: }[87,88]) \text {. Stem: Used to treat cough and cold } \\
\text { (Taplejung: }[87,88]) \text {. }\end{array}$ \\
\hline 656 & Schima wallichii (DC.) Korth. & Tree & Theaceae & Chilone (Np); Sakriphal (S) & $900-2100$ & $\begin{array}{l}\text { Bark: Crushed with common salt and used as vermicide in cattle (Sikkim: [97]). } \\
\text { Rubbed on caterpillar infected portion to remove hair (Sikkim: [11]). Pounded } \\
\text { and given to cure fever and stomach pain, and also applied in bone fracture } \\
\text { and sprain (Jhapa: [66]). Bark paste with the bark of Bridelia retusa applied } \\
\text { externally on deep cuts and wounds (Sikkim: [79]). }\end{array}$ \\
\hline 657 & Aquilaria malaccensis Lam. & Tree & Thymelaeaceae & Aagurushing $(\mathrm{Dz} / \mathrm{Sh} / \mathrm{T})$ & & $\begin{array}{l}\text { Stem: Heartwood used as medicine (KL Bhutan: [71]) and incense (KL Bhutan: } \\
\text { [70]). }\end{array}$ \\
\hline 658 & $\begin{array}{l}\text { Daphne bholua Buch.- } \\
\text { Ham.ex D.Don }\end{array}$ & Shrub & Thymelaeaceae & $\begin{array}{l}\text { Tunguma (Li); Baruwa, Kagaj Pate, } \\
\text { Lokta, Seto Baruwa, Seto Lokta, Sikre } \\
\text { (Np); Sugumendo, Da Mendok (Sh); Da } \\
\text { Mendok (Wi). }\end{array}$ & $2000-4000$ & $\begin{array}{l}\text { Roots and bark: Used to treat intestinal worms; decoction used in fever } \\
\text { (Taplejung: [87]). Bark: Used in the manufacture of handmade paper (KL Nepal: } \\
\text { [83]; KL Bhutan: [93]). }\end{array}$ \\
\hline 659 & $\begin{array}{l}\text { Daphne papyracea Wall. ex } \\
\text { Steud }\end{array}$ & Shrub & Thymelaeaceae & $\begin{array}{l}\text { Tunguma (Li); Aule Lokti, Dangma, } \\
\text { Jhapre Lokta, Kagatey, Kalo Baruwa } \\
\text { (Np); Khultum (Ri) }\end{array}$ & $1500-2400$ & $\begin{array}{l}\text { Roots: Used for intestinal troubles (Sikkim: Database). Decoction given in food } \\
\text { poisoning (Sikkim: [79]; Darjeeling: [91]; KL Nepal: [83]). Used as a substitute of } \\
\text { Aconitum sp. (Sikkim: [79]). Bark: Decoction given to treat fever (Sikkim: } \\
\text { Database). Juice used to treat intestinal worms and used as anti-leech agent; } \\
\text { powder cause nasal irritation (Taplejung: [87]). Used to make handmade paper } \\
\text { (KL Nepal: [83]; Sikkim: [1 1]). Leaves: Given to baby goat during diarrhea and } \\
\text { fever; stalks used to make locally used mats (Sikkim: [11]). }\end{array}$ \\
\hline 660 & Daphne sp. & Shrub & Thymelaeaceae & & & Inner wood: Used as incense (KL Bhutan: [70]). \\
\hline 661 & $\begin{array}{l}\text { Edgeworthia gardneri (Wall.) } \\
\text { Meisn. }\end{array}$ & Shrub & Thymelaeaceae & $\begin{array}{l}\text { Nepalese paper bush (Eg); Argeli, } \\
\text { Argaily (Np) }\end{array}$ & 1500-3000 & $\begin{array}{l}\text { Bark: Used to make paper (KL Bhutan: [93], KL Nepal: [83]; Sikkim: [72]; } \\
\text { Database). Bark: Used as fish poison (Sikkim: Database). }\end{array}$ \\
\hline 662 & Trapa bispinosa Roxb. & Herb & Trapaceae & Paniphalz (S); Paniphal singara (Np). & $200-600$ & Fruit: Edible (Jhapa: [92]). \\
\hline 663 & Trema orientalis (L). Blume & Tree & Ulmaceae & Sitaundu (S); Khari (Np) & $1000-1200$ & Fruit: Edible (Jhapa: [92]). \\
\hline
\end{tabular}


Table 1 NTFPs used by the local people of the Kangchenjunga Landscape, Eastern Himalaya (Continued)

\begin{tabular}{|c|c|c|c|c|}
\hline 664 & $\begin{array}{l}\text { Dendrocnide sinuata } \\
\text { (Blume) Chew }\end{array}$ & Shrub & Urticaceae & Morange (Np) \\
\hline 665 & $\begin{array}{l}\text { Elatostema lineolatum } \\
\text { Wight }\end{array}$ & Shrub & Urticaceae & Damroo (Bhu) \\
\hline 666 & $\begin{array}{l}\text { Elatostema platyphyllum } \\
\text { Wedd. }\end{array}$ & Shrub & Urticaceae & Gagleto (Li) \\
\hline 667 & $\begin{array}{l}\text { Girardinia diversifolia (Link) } \\
\text { Friis }\end{array}$ & Herb & Urticaceae & $\begin{array}{l}\text { Himalayan Nettle (Eg); Mayu Sagi (Li); } \\
\text { Kuju (Lp); Allo (Np); Ptale (Ri) }\end{array}$ \\
\hline 668 & $\begin{array}{l}\text { Girardinia palmata (Forssk.) } \\
\text { Gaudich. }\end{array}$ & Shrub & Urticaceae & Zochha (Dz); Gomjazu (Sh); \\
\hline 669 & Girardinia sp. & Herb & Urticaceae & Sissnu (Np) \\
\hline 670 & Laportea terminalis Wight & Herb & Urticaceae & Sishnu (Np); Sorong (L) \\
\hline 671 & Urtica dioica L. & Herb & Urticaceae & $\begin{array}{l}\text { Stinging Nettle (Eg); Polo (Gr, Tm); Sagi } \\
\text { (Li); Sisnu (Np); Sajilim, Sokhima (Ri); } \\
\text { Sadukpa (WI) }\end{array}$ \\
\hline 672 & $\begin{array}{l}\text { *Nardostachys grandiflora } \\
\text { DC. }\end{array}$ & Herb & Valerianaceae & $\begin{array}{l}\text { Spikenard (Eng); Pagbon, Pangbo, } \\
\text { Pangbwang (Li); Pangpay (Dz/Sh); } \\
\text { Bhulte, Bhutle, Jatamasi (Np); Bhultya } \\
\text { (Np-DI); Ngorochi (Ri); Jatamansi (Sn); } \\
\text { Pangboe (WI) }\end{array}$ \\
\hline 673 & Valeriana hardwickii Wall. & Herb & Valerianaceae & Nakkali Jatamansi (Np) \\
\hline 674 & *Valeriana jatamansii Jones & Herb & Valerianaceae & $\begin{array}{l}\text { Indian Valerian (Eg); Nappu (Gr-Mn); } \\
\text { Samayo, Sugandhawal (Np); Bhutakesi } \\
\text { (Sn); Jaboe (WI) }\end{array}$ \\
\hline 675 & Callicarpa arborea Roxb. & Tree & Verbenaceae & Guahelo (Np) \\
\hline 676 & Callicarpa macrophylla Vahl & Shrub & Verbenaceae & $\begin{array}{l}\text { Guenlo, Sumali (Np); Tichangsa (Che); } \\
\text { Dahidhula (Dar); Dahigun (Tha) }\end{array}$ \\
\hline 677 & Gmelina arborea Roxb. & Tree & Verbenaceae & Khamari (Np); Kasnar (S) \\
\hline
\end{tabular}

200-1000

200-1800

$700-1900$

$1700-3000$

Himalayan Nettle (Eg): Mayu Sagi (Li);

(n)
Roots: Juice taken in constipation (llam: [73, 76]). Young shoots: Eaten as green vegetable (Ilam: [94]; Taplejung: [87]; KL Nepal: [83]). Used as substitute for dal which is good for diabetes (Darjeeling: [80]). Leaves: Paste given in headache and joint pain (llam: [76]). Flowers: Taken to treat blood pressure (Sikkim: [75]).

Inflorescence: Edible (KL Bhutan: [70]).

Bark: Used to make ropes (KL Bhutan: [100]).

1900-3300

$1000-4000$

Leaves and flowers: Used in blood pressure complaints (Sikkim: [75]).

Plant: Juice used to treat jaundice, toothache, and hematuria (llam: [73, 76]). Roots: Paste applied as antidote to dog bite and on minor fractures. Young shoots: Taken as vegetables (Darjeeling: [80]; Taplejung: [88]; Sikkim: [1 1], Database). Young shoots and inflorescences cooked and given in hypertension (Darjeeling: [52]). Leaves: Cooked and taken to maintain blood pressure (Darjeeling: [80]; Sikkim: [89]).

3200-5300 Whole plant and roots: Useful in skin diseases, leprosy, ulcers, and cough. Roots: Used for incense and also in wounds, cough, cold, chronic fever, high blood pressure, and stomach diseases (Sikkim: [49]; Taplejung: [87]; KL Nepal: [83]). Infusion taken to treat stomachache and as laxative (Sikkim: $[75,85]$ ). Decoction used in fever (Darjeeling: [49, 78]). Juice or decoction taken in Decoction used in fever (Darjeeling: [49, 78]). Juice or decoction taken in
dysentery and constipation (llam: [76]). Used as tonic, stimulant, antispasmodic diuretic and antiflatulent (Sikkim: [69]). Used as medicine (KL Bhutan: [71]) and diuretic and antiflatulent (SkK:
incense (KL Bhutan: [70]).

1200-4000 Roots: Given in hysteria, epilepsy, and neurosis (Sikkim: [75]). Used as carminative and stimulant (Sikkim: Database). Extract taken to cure urine trouble (Sikkim: [11]).

1200-3600 Rhizome: Used in fever, cold (Taplejung: [82]), headache, eye compliants, sore throat, wounds, and indigestion (KL Nepal: [83]). Dried rhizome also used as incense (KL Nepal: [83]). Juice given in hysteria, epilepsy, cholera, cough, asthma, weakness and hairfall (llam: [76]).

$1000-2500$

Bark: Juice given to treat fever (Sikkim: Database). Roots: Chewed in cases of boils on the gums (Sikkim: Database). Fruit: Juice administered to cure fever (Sikkim: [79]).

$300-1500$

Roots: Used to treat pneumonia (Panchthar: [67]). Decoction drunk to cure bronchitis (Jhapa: [68]). Bark: Used in rheumatism and gonorrhea (Sikkim: Database).

200-1100 Bark: Pounded and taken as antidote for all kinds of poison; also used in dysentery (Jhapa: [66]). 
Table 1 NTFPs used by the local people of the Kangchenjunga Landscape, Eastern Himalaya (Continued)

\begin{tabular}{|c|c|c|c|c|c|c|}
\hline 678 & Lantana camara L. & Shrub & Verbenaceae & & & $\begin{array}{l}\text { Leaves: Juice applied in cut to heal; crushed and tied over the sprain to } \\
\text { relieve pain (Sikkim: }[11,79]) \text {. }\end{array}$ \\
\hline 679 & Premna serratifolia L. & Shrub & Verbenaceae & Gineri (Np) & & Roots: Decoction taken as laxative and carminative (Sikkim: [85]). \\
\hline 680 & Vitex negundo L. & Shrub & Verbenaceae & Simali (Np); Sindware (S) & $200-1400$ & $\begin{array}{l}\text { Plant: Juice used to treat fever, ulcer, asthma, and cough (Illam: [73]). Leaves: } \\
\text { Used to treat sinusitis and rheumatism (Panchthar: [67]); also used as repellent } \\
\text { for insects and bedbugs (Jhapa: [66]). Stem: Juice bath taken to treat body } \\
\text { swelling, common cold and influenza (Darjeeling: [74]). }\end{array}$ \\
\hline 681 & Viola biflora L. & Herb & Violaceae & Ghattey Ghans (Np) & $2100-4500$ & Roots and flowers: Used as emetic and antiseptic (Sikkim: [75]). \\
\hline 682 & Viola diffusa Ging. ex DC. & Herb & Violaceae & Ghattey Ghans (Np) & $1400-2000$ & Flowers: Taken to get relief from chest pain (Sikkim: [75]) \\
\hline 683 & Viola serpens Wall. & Herb & Violaceae & Ghattejhar (Np) & & Roots: Juice taken in fever (Darjeeling: [78]). \\
\hline 684 & $\begin{array}{l}\text { Ampelocissus barbata (Wall.) } \\
\text { Planch. }\end{array}$ & Climber & Vitaceae & Jarila lahara (Li) & & $\begin{array}{l}\text { Plant: Juice given to treat sores in mouth and tongue of milk sucking baby } \\
\text { (Sikkim: [79]). }\end{array}$ \\
\hline 685 & $\begin{array}{l}\text { Ampelocissus latifolia } \\
\text { (Roxb.) Planch. }\end{array}$ & Climber & Vitaceae & Icewar (S) & $300-1600$ & Fruits: Edible (Jhapa: [92]). \\
\hline 686 & $\begin{array}{l}\text { Ampelocissus sikkimensis (M. } \\
\text { A. Lawson) Planch. }\end{array}$ & Climber & Vitaceae & & $1000-2000$ & $\begin{array}{l}\text { Plant: Juice used to cure sores in the mouth of an infant and treats foot and } \\
\text { mouth disease in cattle (Sikkim: [11]). }\end{array}$ \\
\hline 687 & $\begin{array}{l}\text { Alpinia allughas (Retz.) } \\
\text { Roscoe }\end{array}$ & Herb & Zingiberaceae & Churampha (Np) & $400-600$ & Rhizomes: Extract taken in diabetes and as laxative (KL Nepal: [53]) \\
\hline 688 & Amomum subulatum Roxb. & Herb & Zingiberaceae & Bada alaichi (Li) & $500-2000$ & $\begin{array}{l}\text { Seeds: Used as medicine (KL Bhutan: [71]). Used in indigestion and vomiting } \\
\text { (llam: [76]).Decoction used to gargle to treat teeth and gum infection (Sikkim: } \\
[79,91]) \text {. Oil applied on eye-lids to allay inflammation of the eye in cattle; paste } \\
\text { applied externally as antidote for scorpion-sting and insect bites (Sikkim: [84]). }\end{array}$ \\
\hline 689 & $\begin{array}{l}\text { Caulokaempferia sikkimensis } \\
\text { (King ex Baker) K. Larsen }\end{array}$ & Herb & Zingiberaceae & Bhuin Champa (Li) & & $\begin{array}{l}\text { Bulb: Poultice from crushed bulbs applied to heal fractured bone and wound } \\
\text { (Sikkim: [11]). }\end{array}$ \\
\hline 690 & $\begin{array}{l}\text { Costus speciosus (Koenig) } \\
\text { Sm. }\end{array}$ & Herb & Zingiberaceae & Bet Lauri (Np); Urat (S) & 400-700 & $\begin{array}{l}\text { Roots: Useful in fever, bronchitis, anemia, rheumatism and diabetic (Darjeeling: } \\
\text { [78]; Sikkim: [69]) and diabetic (Darjeeling: [51, 78]; Sikkim: [96]).Used as } \\
\text { antiinflamatory, stimulant and anthelmintic and also given in urinary stones } \\
\text { (Sikkim: [69]). Juice taken before breakfast to cure urinary tract infection; juice } \\
\text { mixed with sugar and used to treat veneral disease (Sikkim: [11]). Juice mixed } \\
\text { with milk and sugar cubes and drunk to cure sensation of internal heat and } \\
\text { stomach inflammation (Jhapa: [66]). Roots of this plant grounded with leaves } \\
\text { of Swertia chirayita and taken in fever and urinary complaints (Jhapa: [66]). } \\
\text { Root mixed with leaves of Drymeria cordata androot paste of Bombax ceiba } \\
\text { and combinely crushed and administered orally to treat urinary disorders } \\
\text { (Sikkim: [79]). Rhizome powder given to cattle to treat fever and inflammation } \\
\text { (Sikkim: [84]). Stem: Extract given in diabetes and cholera (KL Nepal: [53]). }\end{array}$ \\
\hline 691 & Curcuma angustifolia Roxb. & Herb & Zingiberaceae & $\begin{array}{l}\text { Bakhre Saro (Np); Nauhaine- haldai } \\
\text { (Me) }\end{array}$ & $100-1500$ & $\begin{array}{l}\text { Rhizomes: Powder used as antiseptic in cuts, wounds and to check bleeding } \\
\text { (Jhapa: [68]). }\end{array}$ \\
\hline 692 & Curcuma aromatica Salisb. & Herb & Zingiberaceae & $\begin{array}{l}\text { Wild turmeric (Eg); Bandhale, } \\
\text { Banhaledo, Kalohaledo (Np); Vanharida } \\
\text { (Sn) }\end{array}$ & 700-1100 & $\begin{array}{l}\text { Rhizomes: Used in cough and bronchitis (Taplejung: [82]). Also used as } \\
\text { appetizer, tonic, carminative and applied to bruises and sprains; powder used } \\
\text { as anthelmintic; oil useful in the treatment of early stage of cervix cancer } \\
\text { (Sikkim: [69]). }\end{array}$ \\
\hline
\end{tabular}


Table 1 NTFPs used by the local people of the Kangchenjunga Landscape, Eastern Himalaya (Continued)

693 Curcuma caesia Roxb. Herb Zingiberaceae $\quad$ Kalo haledo (Np) 200-1500

694 Curcuma longa L.

Herb

Zingiberaceae

Hardi (Np); Juung (Sh); Yongka (Dz)

695 Curcuma zeodaria Rosc

Herb

Zingiberaceae Sh)

696 Elettaria cardamomum (L.) Herb Maton

697 Hedychium spicatum Sm. Herb

698 Kaempferi agalanga L. Herb

699 Kaempferia rotunda L. Herb

700 Zingiber cassumunar Roxb.

Herb

Zingiberaceae

Banada (Np)

701 Zingiber officinale Rosc

Zingiberaceae

Aduwa (Np)

Fungi

1 Lycoperdon pyriforme Schaeff.

$2 \quad{ }^{*}$ Ophiocordyceps sinensis (Berk.) Sung et al.

Agaricaceae

Ophiocordycipitaceae

\section{Lichen}

1 Usnea logissima Ach.

2 Usnea sikkimensis Biswas
Usneaceae

Usneaceae ichen, Old-man's beard ( $\mathrm{Eg})$ : Jhyau 2500-3900 (Np) alo Hardi (Np); Zalmathangru- tsalu

Rhizomes: Powder taken to treat leucoderma, piles, bronchitis, and asthma (llam: [73, 76]; Sikkim: [69]). Fresh rhizomes eaten raw to expel gas (Sikkim: [11]). Used as stomachic, diuretic, aromatic, stimulant, carminative and to cure sprains and bruishes (Sikkim: [69]).

Rhizomes: Used as medicine (KL Bhutan: [71]). Paste prepared from powder and applied on the fractured bones (Darjeeling: [52]).

Rhizomes: Used as medicine (KL Bhutan: [71]).Eatern raw to cure diarrhea colic and indigestion; paste applied to treat skin diseases (Sikkim: [11]). Decoction taken to get rid from fever (Darjeeling: [78]). Cut into small pieces and administered orally to treat stomach pain, loss of appetite and also used as antidote to food poisonong (Darjeeling: [52]).

Used as medicine (KL Bhutan: [71]). Fruit: Used as spices (KL Bhutan: [70]).

Rhizomes: Used as medicine (KL Bhutan: [71]). Taken to treat diarrhea, vomiting, and asthma (Sikkim: $[69,75])$.Used in liver disorders and stomach ailments (Sikkim: [69]).

Rhizomes: Used as medicine (KL Bhutan: [71]).

1300-2000 Plant: Juice used in gastric problems, tumors, ulcers, wounds (llam: [73, 76]) and swelling, and wounds (llam: [76]). Tubers: Used as bone settlers (Sikkim: [69], Database) and as poultice in fracture, healing fresh wounds, and removes coagulated bloods from the body (Darjeeling: [80]). Paste prepared along with the roots of Laportea terminalis and aerial portion of Viscum albumand applied on bone fracture and dislocated joints (Darjeeling: [52]). Root decoction used analgesic and sedative (Darjeeling: [74]).

Leaves and inflorescence: Given in cough and fever, dyspepsia. Inflorescence heated in fire and sniff against sinusitis (Darjeeling: [69]). Rhizomes: Soup taken during stomachache (KL Nepal: [53]).

Rhizomes: Used as medicine (KL Bhutan: [71]). Used as laxative, aphrodisiac, carminative, also useful in heart diseases, throat, and asthma (Sikkim: Database). Used as spice; juice and powder used to cure joint pain, headache, cough, and cold (llam: $[73,76])$.Roasted rhizome chewed with salt during severe dry cough (Sikkim: [79]).

Whole plant: Used as medicine (KL Bhutan: [71]).

Plant: Used as medicine (KL Bhutan: [71]). Rejuvenates liver, heart and cheeks ageing process and built up immune system (Sikkim: Database). Species has high commercial value (Bhutan: [99]; Sikkim: [98]). Known to treat health complaints like aging, cancer, diabetes, fatigue, heart diseases, lungs and liver ailments (Sikkim: [96). Taken as aphrodiasic (KL Nepal: [53).

Thallus: Useful in wounds, lung, liver, and fever from poisoning: also useful as incense (KL Nepal: [83]).

Thallus: Used to bandage surface of wound and skin eruptions; inserted into the nose to stop bleeding (Sikkim: [11]). 
Table 1 NTFPs used by the local people of the Kangchenjunga Landscape, Eastern Himalaya (Continued)

\section{$\frac{\text { Table } 1}{\text { Algae }}$}

1 Ulva spp.

\section{Pteridophytes}

1 Adiantum capillus-veneris L. Herb

2 Diplazium asperum Blume Herb

3 Diplazium esculentum Herb (Retzius) Swart

4 Diplazium laxifrons Rosenstock

5 Diplazium maximum (D. Herb Don) C. Christensen

6 Dryopteris fragrans (L.) Herb Schott

7 Equisetum diffusum D. Don Herb

8 Equisetum ramosissimum Herb subsp. debile (Roxb. ex Vaucher) Hauke

$9 \quad$ Lycopodium clavatum L

Nephrolepis cordifolia (L.) C. Herb Pres

11 Lepisorus thunbergianus Herb (Kaulf.) Ching

12 Drynaria quercifolia (L.) J. Herb Smith

13 Aleuritopteris leptolepis Herb (Fraser-Jenk.) Fraser-Jenk.

14 Pteris biaurita $\mathrm{L}$

5 Pteris terminalis Wallich ex Herb J. Agardh

16 Lygodium flexuosum (L.) Sw. Herb

erb (Algae) Ulvaceae

Adintaceae

Athyriaceae

Athyriaceae

Athyriaceae

Athyriaceae

Dryopteridaceae

Equisetaceae

Equisetaceae

Lycopodiaceae

Naagbeli (Np); Dermusungfon (L)
Whole plant: Edible (KL Bhutan: [70]).

Leaflets: Decoction taken as antipyretic (Darjeeling: [78]).

Roots: Used to cure dysentery (Sikkim: [1 1]).

Fronds: Young fronds cooked and eaten as vegetable (Jhapa: [92]; Darjeeling: [78]; Bhutan: [102].

$100-1200$

$900-1800$

Fronds: Young fronds cooked and eaten as vegetable (Bhutan: [102]).

900-1800

Fronds: Young fronds cooked and eaten as vegetable (Bhutan: [102]).

Stem: Used as medicine (KL Bhutan: [71]).

Plant: Paste applied on bone fracture, sprains, and in urinary troubles (llam: [73]).

Plant: Paste mixed with Drymaria cordata and applied in bone factures (Jhapa: [68]). Aerial parts: Used as clotting agent for wound, nose bleeding, and bleeding of urinary tract (Sikkim: [11]; Database). Paste applied to Cure mouth sores (Darjeeling: [74]).

700-1800 Plant: Administered orally for treatment of muscle contraction in cattle (Sikkim: [84]). Plant and spores: Taken in rheumatism, pulmonary disorders, and chronic kidney. Roots and leaves: Used in treating rheumatism (Sikkim: [75] Database). Spores: Given withpaste of strobilus to cure lung and kidney problems and urinary disorders (llam: [73, 76, 77]). Also used to treat scabies and ringworms (Panchthar: [67]). Applied directly on the wound (Sikkim: [79]) Cosumed to treat bleeding after childbirth (Darjeeling: [74]).

Nephrolepidaceae Paniamala $(\mathrm{Np})$

$500-2000$

Polypodiaceae

Polypodiaceae

200-1000

Pteridaceae

Rani sinka (Np)

1000-3000

Pteridaceae

Thado unew (Np)

200-1500

Pteridacea

Nimin Daway (Bhu)

$600-2700$

Schizaceae
Bahun Lahara (Np)
Tubers: Juice taken to treat indigestion, fever, cold, and cough (llam: $[73,76]$ ). Fruit: Edible (llam: $[73,76])$.

Whole plant: Used as medicine (KL Bhutan: [71]).

Rhizomes: Juice with sugar drunk to cure stomach inflammation and sensation of internal heat of cattle; grounded with Oxalis corniculata and applied on bone fracture (Jhapa: [66]).

Plant: Juice used in ulcer and stomachache (llam: [73]).

Stem: Mashed and applied on cuts and wounds to stop bleeding and infection (Sikkim: [11], Database). Frond: Juice consumed to treat dysentery (Darjeeling: [74]).

Young fronds: Cooked as vegetable (KL Bhutan: [102]).

Fronds: Young fronds soaked in mustard oil and applied externally on affected areas to treat rheumatism and sprains (Darjeeling: [74]). 
Table 1 NTFPs used by the local people of the Kangchenjunga Landscape, Eastern Himalaya (Continued)

17 Sphagnum squarrosum Herb

crome

18 Tectaria coadunata (J.Sm.) Herb C.Chr.

\section{Gymnosperm}

1 Ephedra gerardiana Wall ex Shrub Stap

Ephedraceae

Hathijor, Somlata (Np): Kagchalo, Kagcharo, Sallejar (Np-DI); Chhe (Sh), Sankhapa (Sh); Chhewong (Sh, WI), Tshe (Dz)

2 Cupressus spp.

\section{Tree}

Cupressaceae

3 Juniperus communis L.

Tree

Cupressaceae

4 Juniperus indica Bertol.

Tsendenshing (Sh); Tsendey (Dz)

Sukpa (Np)

Black Juniper (Eg); Dhup (Np)

5 Juniperus recurva Buch.- Shrub Ham. ex D.Don

6 Juniperus spp.

Cupressaceae

Cupressaceae

7 Abies densa Griff. ex Parker Tree

Pinaceae

$8 \quad$ Abies spectabilis (D.Don) Tree Mirb.

Pinaceae

Cedrus deodara (Roxb. ex D. Tree Don) G. Don

10 Pinus roxburghii Sarg. Tree

Pinaceae

11 *Taxus wallichiana Zucc. Tree

$3700-4500$

Drooping juniper (Eg); Dhupi (Np); Shup (Dz); Shukpashing (Sh)

Gobre salla (Np); Dungshi (Dz)

Plant: Used as fuel; hunters and graziers use whole moss for dressing wound (Sikkim: [11])

Plant: Boiled and taken as soup for a week to treat dysentery (Darjeeling: [74]).

$500-2500$

$2000-5200$

Plant: Used as medicine (KL Bhutan: [71]). Raises blood pressure and used to relieve high fever (KL Nepal: [83]; Sikkim: Database) and asthma (Sikkim:

Database). Juice used for liver fever, bleeding, and cuts (KL Nepal: [83]. Fruit: Used as digestive (KL Nepal: [83]). Ripe fruits taken to get relief from altitude sickness and indigestion (Sikkim: [79]). Stem: Powder inhaled to treat headache (Sikkim: [79])

Whole plant: Used as incense (KL Bhutan: [70])

Fruits: Dried and chewed as a nut or decoction taken in fever (Darjeeling: [78]).

Leaves and fruits: Used in kidney disorders, skin and lymph disease, fever, cough, and cold, sores, wounds and paralysis of limbs (KL Nepal: [83]). Fruit Powder put on the glowing red fire coal and the scent smelt to relief from headache and reduce blood pressure. Dried fruit powder mixed with tea or milk to treat cough, cold in high altitude (Sikkim: [79]). Leaves: Used as incense (KL Nepal: [83]). 3300-4600 Aerial parts: Used as incense (KL Nepal: [83]). Leaves: Used as medicine (KL

Used as incense (KL Bhutan: [70]).

2550-3700 Leaves: Used as medicine (KL Bhutan: [71]) and incense (KL Bhutan: [70]). Juice taken orally to relieve from stomach pain and fever (Sikkim: [11]). Extract used in asthma, bronchitis, and stomach trouble (Sikkim: [79, 80, 89]). Extract given to cattle to treat fever and loss of appetite (Sikkim: [84]).

Himalayan Silver Fir (Eg); Gobre Salla, Thingre Salla (Np); Thingro (Np-DI); Talispatra (Sn); Som Chirugpa (WI)

Deodara (Np)

Rani Salla (Np); Aang (Li)

Eastern Himalayan Yew (Eg); Silingi (Gr- 2100-3500 $\mathrm{Mn)}$; Dhengre (Li); Lauthsalla (Np); Kandeloti, Lota (Np-DI)

Leaves: Used as incense (Taplejung: $[81,88])$. Leaves and gum: Used as tonic and also useful in tuberculosis and internal hemorrhage (Sikkim: Database). Fruit: Boiled to obtain black ink (Taplejung: [87]).

Oil applied externally to treat ringworm (Sikkim: [97]).

Leaves: Extract used in hydrocele and also during bone fracture (KL Nepal: [53]). Resin: Used as medicine in rheumatism (KL Bhutan: [71, 93]).

Bark: Used as medicine (KL Bhutan: [71]). Taken as herbal tea, especially to treat piles (Taplejung: [87]; KL Nepal: [104]) and in muscular pain and fever. Leaves: Extract used to cure asthma, bronchitis, and other respiratory diseases (KL Nepal: [83]; Ilam: [73, 76]). Leaves: Used as medicine (KL Bhutan: [71]). Antispsmodic, used in nervousness, hysteria and epilepsy (Darjeeling: [69]; Sikkim: [106]). Young shoots used in headache, giddiness, diarrhoea and liver disorders (Darjeeling: [69]; KL Nepal: [104]). Fruits: Eaten raw (KL Nepal: [83]; llam: [73]).

ҒSikkim: Datadase = Medicinal Plant Database of Government of Sikkim:http://www.sikkimforest.gov.in/medicine_main.htm\#tp

National priority herbs of Government of Nepal

Abreviations: Bh Bhotia, Bhu Bhutanese, Dz Dzongkha, Eg English, Gr Gurung, Gr-Mn Gurung of Manang, Km Kham, Li Limbu, Lp Lepcha, M Meche, Np Nepali, Np-D/Nepali in Dolpo, Np-Tp Nepali in Taplejung, Nw

Newari, Ri Rai, S Satar, Sh Sherpa, Sh Sharchop-kha, Sn Sanskrit, Tb Tibetan, Tha Tharu, WI Walung 
additional ailment categories. Chi-square $(x 2)$ was used to test the null hypothesis that there is no difference in use of NTFPs under various use categories among the three countries in the Kangchenjunga Landscape.

\section{Results and discussion}

\section{Pattern of publications}

Majority of publications on NTFP were from India (60\%), while $34 \%$ were from Nepal and $6 \%$ from Bhutan. This is quite obvious as Darjeeling and the state of Sikkim in India make up a large part of the KL (56.3\%). The presence of two state level universities and research institutes has made significant contribution to the research in KL India [29]. Except for Bhutan, the species reported in this study were mostly documented through ethnobotanical studies conducted in different parts of the landscape. A few studies were focused on particular ethnic communities whereas most of the studies were on general ethnobotany of the region with mixed ethnic composition. Publication on NTFPs date back to 1988 in India while in Nepal and Bhutan it was started after 1996 (Fig. 2). However, majority of the publications (86\%) were published after 2000. All publications are qualitative in nature.

\section{Frequency of NTFPs use}

We reported on a total of 739 species of NTFPs used by the local people of Kangchenjunga Landscape. Of these, the highest number of NTFPs was documented from India (377 species), followed by Nepal (363) and Bhutan (245). These numbers, however, overlap in terms of distribution. The NTFPs used only in India were 185 species, while this number was 189 for Nepal and 166 for Bhutan.

\section{Taxonomic diversity and growth habit}

Angiosperms were predominant with 705 taxa belonging to 137 families followed by Gymnosperms (10), Pteridophytes (17), Fungi (3), Lichens (2), Bryophyte (1) and Algae (1). Families with the highest number of species used belong to Asteraceae (56 species), Fabaceae (41), Lamiaceae (27), Rubiaceae (24), Poaceae (23), Moraceae (16), Ranunculaceae (16), Rosaceae (15), Zingiberaceae (15), Polygonaceae (14), Ericaceae (13), Rutaceae (13), and Liliaceae (11). NTFPs were distributed into different life forms, with herbs having the most species followed by trees and shrubs (Fig. 3). Pattern of NTFPs used according to different life forms was similar in Bhutan, India and Nepal. Such herbaceous species were mostly medicinal and their extensive use could be because they were frequently found in the forest, and it is believed that the more abundant a plant is the more medicinal virtues it may possess [30].

\section{Major use categories}

People from Kangchenjunga Landscape used NTFPs for 24 different purposes (Table 2). A comparative analysis revealed that the highest number of use categories were reported from the Kangchenjunga Landscape region of India (20 categories) followed by Nepal (18) and Bhutan (14). Despite common occurrence of many species in India and Nepal, use pattern differed greatly in these two countries. Medicinal plants were among the main valuable NTFPs in the landscape. Of the total NTFPs, 334 species were used in traditional medicinal practice in India, whereas 297 species used in Nepal and 176 species used in Bhutan. A considerable number of species were also used as edibles as fruit, vegetables, and pickles in all three countries (Table 1 and Table 2). Fruit and shoots were the most frequently eaten parts.

The relatively higher number of diversity in wild edible NTFPs in Nepal could be because of higher diversity of ethnic groups living in the lowland Tarai to highland regions. There was a significant difference $(\chi 2=35.06$, $\mathrm{df}=64, \alpha=0.05$ and $1-\alpha=83.67)$ in medicinal plants use pattern in major disease/ailment categories in India and Nepal. These results indicate differences between the traditions of NTFP use in different cultures of India and Nepal. Similar results were also obtained from East Timor [31]. NTFPs use varies from site to site because of the heterogeneity of the community and different traditional practices by ethnic groups [14].

Among 739 species used by the local people, most species were used for a single (550 species) purpose, while fewer were used for two (147) or multiple (42) purposes. Local people were well aware of collecting seasons, mode of collection, and frequency of collection of specific parts of plant species. Medicinal plants such as Heracleum nepalense is plucked on the first Tuesday after the Teej festival. This practice is known as 'Harlo'. The people believe that the medicinal plants plucked on that day are extremely effective and potent [32]. Similar practice of harvesting season can be found among the Amchis of the Himalaya where they believe that for better medicinal efficacy, specific parts of specific medicinal plants should be collected during specific seasons [33].

\section{Ailments treated and preparation methods}

The use of medicinal plant in treatment of particular ailment and the preparation method were not specified from Bhutan. In India and Nepal, a total of 27 major ailments were reportedly treated with medicinal plants with most species being used to treat multiple ailments (Table 3). Gastro-intestinal disorders; fever; cold, cough and sore throat; musculoskeletal disorders; injuries; dermatological infections; respiratory system disorders; nutritional disorders; and poisoning effects were treated with the highest diversity of medicinal plant species (Table 1 and Table 3). The high diversity of species use in gastro-intestinal disorders 
could be because of poor sanitation and drinking water quality in the Kangchenjunga Landscape as in many developing contries [34, 35].

Mode of preparation included juice, paste, decoction, powder, infusion, and chewing raw plant parts (Fig. 4, Table 1). The majority of formulations were prepared as juice followed by paste and decoction. Proper selection of species, parts, as well as preparation and administration methods were very important in traditional health care systems.

Almost all plant parts were used to prepare different medicinal formulations: roots, rhizomes, tubers, bark, leaves, flowers, fruit, seeds, young shoots, whole plants, and gum and latex (Table 1). The most frequently used plant parts were underground parts, followed by leaves, fruit, bark, whole plants, seeds and flowers (Fig. 5). Use of multiple plant parts was often documented (Table 1). The preference for roots and rhizomes to prepare traditional remedies follows the scientific basis that roots generally contain high concentrations of bioactive compounds [36]. Such a trend is also reported from other studies from the Himalaya [35, 37, 38].

\section{NTFPs trade and livelihoods potential}

The role of NTFPs is particularly important in the Himalayan region where a large proportion of the rural population depends on them as a source of medicine, food, fibre, dye and other useful materials [39-41]. In the Kangchenjunga Landscape, many of the NTFPs are used for subsistence, while others are the main or only source of income generation. However, the role of nonmarketed NTFPs that were used for subsistence is largely ignorned when estimates are made of the economic importance of NTFPs to rural populations [42]. Understanding the economic value of non-marketed NTFPs helps to determine the true income of the gatherers and also helps ascertain the true value of the standing forest, leading to more rational decisions about its alternative uses [42].

Domestic as well as cross-border trade of NTFPs, both legally and illegally, is a historical practice in this region [43]. The traded NTFPs mostly include medicinal plants and to a lesser extent some wild edible plants and fibre yielding plants. The handmade paper from Argeli (Edgeworthia gardneri) is the only NTFPs that was sold after value addition in Nepal. Many of the species documented in this study possess high economic potential (Table 4) and could thus supplement family income [44] while generating incentives for biodiversity conservation [45].

Commonly traded NTFPs from the Nepal part of the Kangchenjunga Landscape include medicinal plants such as Dactylorhiza hatagirea, Fritillaria cirrhosa, Neopicrorhiza scrophulariiflora, lichens, and Taxus wallichiana. Other important species under trade are Aconitum species, Valeriana jatamansi, Viscum album and Zanthoxylum species. Species such as Daphne bholua, Edgerworthia gardnerii, Rhododendron anthopogan, Rubia manjith, Swertia chirayita, Valeriana jatamansi, and Zanthoxylum species are traded in large volume following legal procedures. The collected plant materials are normally sold to middlepersons (local traders), with only a few collectors selling or exporting NTFPs directly in local and cross-border markets. The total amount of NTFPs traded from Nepal in the last five years was 2,029,960 kg and the amout of revenue generated was around US\$ 76,066 (Fig. 6, Table 4). The lack of openly accessible information on traded species of NTFPs from Bhutan and India limited our ability to conduct a comparative analysis.

Despite the high potential for trade and livelihoods through NTFPs, local people in the Kangchenjunga Landscape are not able to adequately benefit from engaging in the NTFP sector. In most cases, collectors were not aware of the market price for their products and

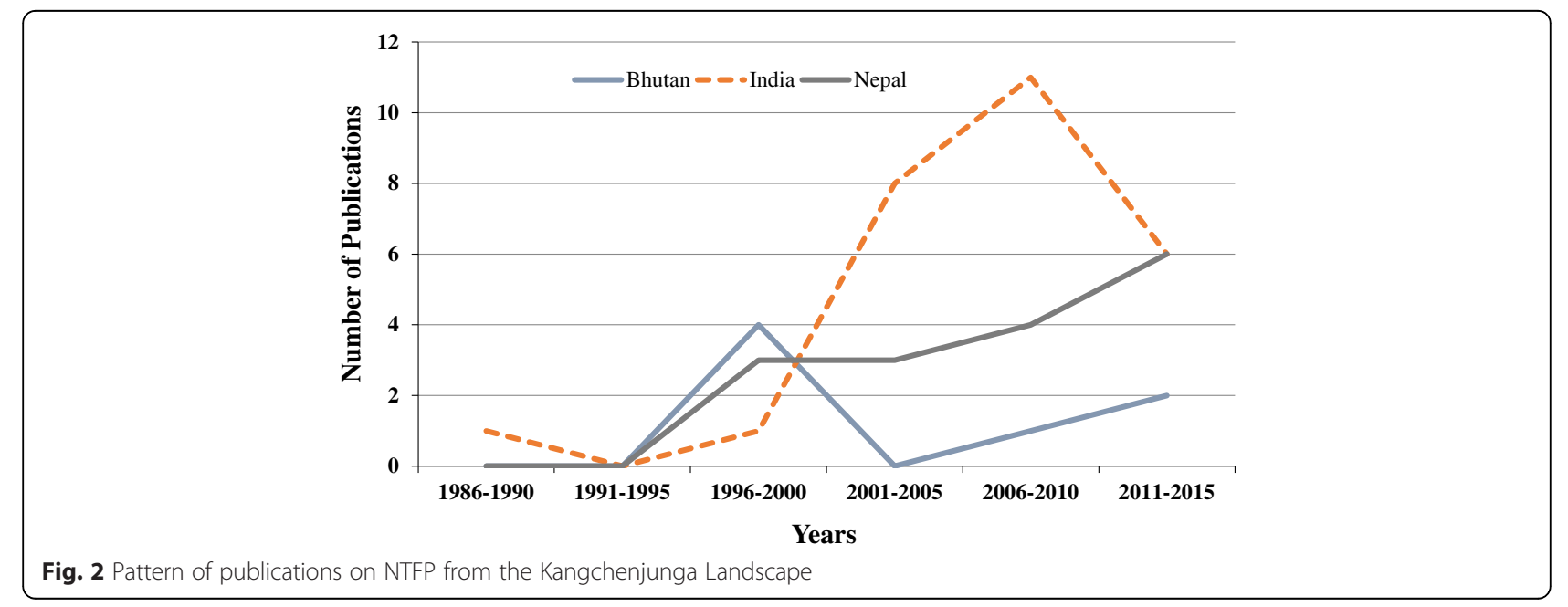




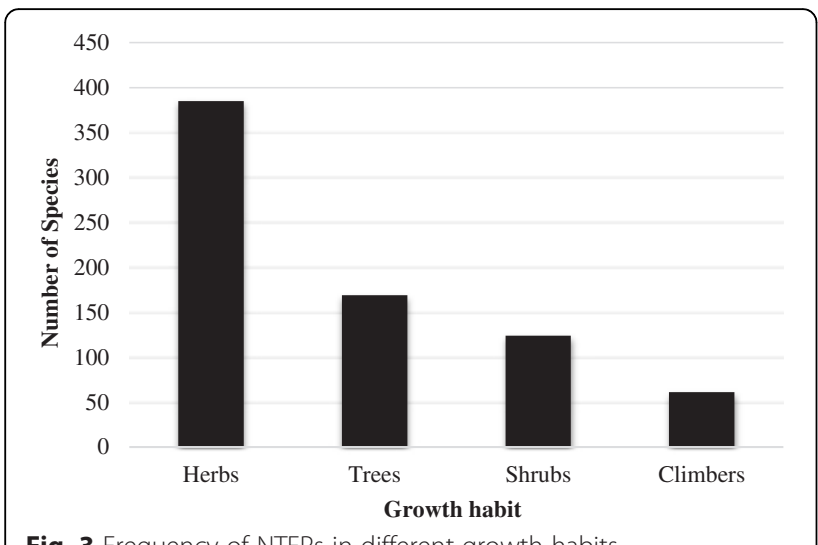

Fig. 3 Frequency of NTFPs in different growth habits

were compelled to sell based on the offers of the middlepersons [46]. Thus, ensuring that market information is available to local people is one of the challenges in the NTFP sector in the landscape. Moreover, traders reported several other issues including multiple taxation system,

Table 2 Major use categories of NTFPs and frequency of taxa reported from Kangchenjunga Landscape

\begin{tabular}{|c|c|c|c|c|}
\hline \multirow[t]{2}{*}{ Use category } & \multicolumn{3}{|c|}{ Frequency of taxa reported } & \multirow{2}{*}{$\begin{array}{l}\text { Total } \\
\text { (Kangchenjunga } \\
\text { Landscape) }\end{array}$} \\
\hline & Bhutan & India & Nepal & \\
\hline Basket & 4 & 3 & - & 7 \\
\hline Broom & 2 & - & 1 & 3 \\
\hline Cigarette wrapper & - & - & 1 & 1 \\
\hline Detergent & - & 1 & - & 1 \\
\hline Dye & 12 & 6 & 6 & 23 \\
\hline Edible & 62 & 46 & 130 & 191 \\
\hline Fencing & 2 & 1 & 1 & 4 \\
\hline Fermentation & - & 4 & 3 & 4 \\
\hline Fibre & 10 & 4 & 4 & 15 \\
\hline Fish poisoning & - & 1 & 1 & 2 \\
\hline Fodder & 3 & 4 & 12 & 19 \\
\hline Fuel & - & 1 & 1 & 2 \\
\hline Gum & 4 & - & - & 4 \\
\hline Incense & 19 & 3 & 13 & 33 \\
\hline Insecticide & - & 2 & 1 & 3 \\
\hline Medicinal & 176 & 334 & 297 & 598 \\
\hline Oil & 6 & 2 & 7 & 7 \\
\hline Ornamental & 5 & 1 & - & 2 \\
\hline Preservator & - & 1 & - & 1 \\
\hline Roofing & 2 & - & - & 2 \\
\hline Ritual & - & 3 & 5 & 8 \\
\hline Spritual & - & 1 & 1 & 2 \\
\hline Tea & 1 & 4 & 4 & 8 \\
\hline Vetenery medicine & - & 27 & 5 & 27 \\
\hline
\end{tabular}

hurdles during transportation, and duration of transport permit.

As reported by Sundriyal and Sundriyal [47] from Sikkim within the Kangchenjunga Landscape, the sale of fruit provides minimum returns due to fairly low shelf life and market costs. Therefore, some value addition in the form of pickle, chutney, jam, jelly, etc. may increase fruit shelf-life and economic profit to local communities. This reflects a clear need to diversify the product base and to ensure that wild edible plants fetch higher prices [47]. There is also need for value addition for other NTFPs. Therefore, value addition at the local level is an essential part of NTFP trade. Untapped but potential species of NTFPs such as wild edible fruit and vegetables could be promoted in local markets. These could also be promoted for visitors in hotels and restaurants.

Another major problem in commercialization of NTFPs is the low volume in which they are collected and produced, in contrast to the large quantities that are required for the markets. This problem could be addressed by establishing cooperatives, and using these cooperatives for collective marketing which will ensure optimum benefits to collectors [48].

\section{Threats and conservation challenges}

Unustainable harvesting of NTFPs, mostly medicinal and edible plants, is the major threat to conservation and management of NTFPs in the Kangchenjunga Landscape $[47,49]$. Sustainable harvesting is essential for conservation of NTFPs, and in turn for ensuring the livelihoods of many rural peoples. Indeed, promotion of commercial extraction of NTFPs as a conservation strategy is based on the argument that forest conservation must be able to offer economic incentives to local peoples in order to counter the threat from destructive land uses such as logging and grazing. This strategy has gained wide acceptance as a conservation paradigm [2]. As indicated by Ticktin [2], despite growing concern over the conservation of these species, as well as their potential to enhance forest conservation and livelihoods, information on the ecological implications of harvest is not available in the Kangchenjunga Landscape.

Illegal trade of NTFPs from the landscape often includes some of the CITES Appendix listed species such as Sunakhari (Orchids), Kutki (Neopicrorhiza scrophulariflora), and Lauth salla (Taxus wallichiana). The trade also includes some plant species under legal protection of the Government of Nepal like Orchids, Champ (Michelia champaca), Jhyau (Lichens), Jatamansi (Nardostachys grandiflora), and Sughandhawal (Valeriana jatamansi) [43]. These species are mostly traded to India via local collectors, whereas limited quantity of these items are exported to Tibet [50, 51]. Conserving such species is challenging, yet illegal trade has 
Table 3 Major disease categories and number of taxa reported from Kangchenjunga Landscape

\begin{tabular}{|c|c|c|}
\hline \multirow[t]{2}{*}{ Disease/ailment/condition category } & \multicolumn{2}{|c|}{$\begin{array}{l}\text { Number of } \\
\operatorname{taxa}^{a}\end{array}$} \\
\hline & India & Nepa \\
\hline Blood system disorders (purification, anaemia, etc.) & 9 & 9 \\
\hline $\begin{array}{l}\text { Circulatory system disorders (heart problems, blood } \\
\text { pressure, etc.) }\end{array}$ & 23 & 9 \\
\hline Cough, cold and sore throat & 84 & 54 \\
\hline $\begin{array}{l}\text { Dermatological infections (boils, eczema, itch, } \\
\text { leucoderma, leprosy, running sore, dropsy, irritant, } \\
\text { small pox, chicken pox, skin problems, etc.) }\end{array}$ & 86 & 63 \\
\hline Diabetes & 19 & 11 \\
\hline Earache, ear irritation & 3 & 5 \\
\hline Fainting and fits & - & 2 \\
\hline Fever/malarian fever & 83 & 64 \\
\hline $\begin{array}{l}\text { Gastro-intestinal disorders (bile disorder, cholera, colic, } \\
\text { constipation, indigestion, diarrhoea, dysentery, dyspepsia, } \\
\text { emetic, laxative, liver disorders, piles, purgative, stomach } \\
\text { pain, ulcer, intestinal worms, vomiting, etc.) }\end{array}$ & 312 & 219 \\
\hline General health (alterative, antiperiodic, prophylactic, etc.) & 7 & 5 \\
\hline $\begin{array}{l}\text { Gynaecological problems (menstrual disorders, pain, } \\
\text { vaginal and uterine problems, etc.) }\end{array}$ & 8 & 12 \\
\hline Haemorrhages (internal bleeding, nasal haemorrhage, etc.) & 2 & 2 \\
\hline Hair care (prevent hair loss, scalp problems, lice) & 7 & 8 \\
\hline Headache & 18 & 20 \\
\hline Injuries (cuts and wounds, burns) & 65 & 54 \\
\hline $\begin{array}{l}\text { Mental disorders (Hysteria, insomnia, seizures, } \\
\text { nervousness, etc.) }\end{array}$ & 23 & 10 \\
\hline $\begin{array}{l}\text { Musculoskeletal disorders (analgesic, arthritis, gouts, } \\
\text { bone facture, rheumatism, body pain, joint pain, sprains, } \\
\text { swellings, cramps, muscle relaxant, etc.) }\end{array}$ & 76 & 84 \\
\hline Nervous system disorders (paralysis, hypertenson, etc.) & 5 & 4 \\
\hline Nutritional disorders (weight loss, tonic, appetizers, etc.) & 42 & 17 \\
\hline $\begin{array}{l}\text { Odontological problems (tooth ache, gum problems, } \\
\text { decayed teeth) }\end{array}$ & 17 & 15 \\
\hline $\begin{array}{l}\text { Ophthalmological disorders (eye wash, sore eyes, } \\
\text { infection, etc.) }\end{array}$ & 13 & 12 \\
\hline $\begin{array}{l}\text { Poisoning (insect bites, leech bites, rabies, snake bites, } \\
\text { bee stings, food intoxication) }\end{array}$ & 25 & 26 \\
\hline $\begin{array}{l}\text { Pregnancy, child birth, puerperium (labour induction, } \\
\text { labour pain, after child birth, miscarriages, abortion, } \\
\text { lactation stimulant, pregnancy prevention) }\end{array}$ & 8 & 17 \\
\hline $\begin{array}{l}\text { Respiratory system disorders (asthma, bronchitis, plague, } \\
\text { chest pain, expectorant, pneumonia, tuberculosis, altitude } \\
\text { sickness, nasal irritation) }\end{array}$ & 65 & 55 \\
\hline Sexual health/dysfunction & 7 & 8 \\
\hline $\begin{array}{l}\text { Urinary system disorders (hematuria, kidney, urination, } \\
\text { diuretic etc.) }\end{array}$ & 26 & 27 \\
\hline Venereal diseases (gonorrhea, spermatorrhea etc.) & 7 & 11 \\
\hline
\end{tabular}

${ }^{a}$ Most taxa were reported to be used in more than one disease/ailment/ condition category (see Table 1) slightly decreased in the last decade due to effective conservation efforts of local organizations and increased cultivation practices in the landscape. Community forestry, which has restricted open access to NTFPs, and resource monitoring have also been effective in conserving NTFPs in recent years. In addition, availability of economically important NTFP species has currently declined due to deforestation and replacement with monoculture, use of pesticides and over harvesting [50]. Traditional knowledge on the use of NTFPs such as medicinal plants is also gradually declining due to socio-economic transformation in the Kangchenjunga Landscape [49, 52, 53].

Monitoring is one of the key components to promote the NTFP sector. Follow-up of rules, regulations and strategies related to NTFPs is necessary for contributing to changes in policy that are able to mainstream sustainable management of NTFPs with livelihoods improvement. Limited progress has been achieved in the Kangchenjunga Landscape in controling over-harvesting, enforcing effective harvesting regimes, and maintaining conducive and adaptive adminstrative processess. Recently adopted economic tools such as certification of sustainable harvests should also be applied as a means of ensuring that NTFPs collected sustainably can be identified as such by the consumers [54, 55].

NTFPs reported from the Kangchenjunga Landscape also include many species under different threat categories as well as under priorities of the governments. For example, of the total 30 national priority herbs of Nepal, 26 are abundantly available in the Kangchenjunga Landscape, while all species prioritized for cultivation and research in Nepal are also reported from the Landscape [56]. Among these, Nardostachys grandiflora, Neopicrorhiza scrophulariiflora, Rauvolfia serpentina and Taxus wallichiana are the most threatened species. Therefore, the economic, socio-cultural and conservation values of these NTFPs are extremely high.

\section{NTFP policy frameworks}

A comparision of NTFP policy frameworks in the Kangchenjunga Landscape shows that Bhutan, India and Nepal have supportive policies for the NTFP sector, thereby providing enabling environments and support for NTFP programs and marketing [25]. As a result, many development agencies including national and international non-governmental organizations have placed emphasis on NTFPs in their programs. The collection, conservation and sustainable utilization of NTFPs in Bhutan is mostly guided by the National Strategy for the Development of Non-Wood Forest Products 2008-2018. Other sectoral policies are the Forest Act 1969, Plant Quarantine Act of Bhutan 1993, Forest and Nature Conservation Act of Bhutan 1995, Environmental Assessment Act 2000 and 


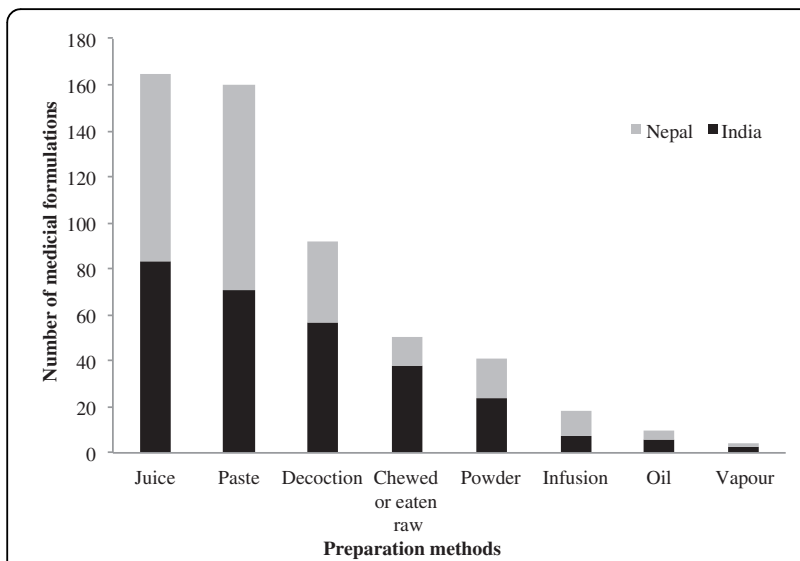

Fig. 4 Use frequency (number of medicinal formulations) of different remedy preparation methods in India and Nepal

Biodiversity Act and Framework of Bhutan 2003, 2006 [57]. The Indian National Forest Policy (1988) makes a special mention of NTFPs emphasizing on protection, improvement and their enhanced production for generation of employment and income [58]. Likewise, in Nepal there are several sectoral as well as specific policy provisions for sustainable use and management of NTFPs $[15,55]$. The most comprehensive policy is the Herbs and Non-Timber Forest Product Development Policy 2004 [59]. The recent Nepal National Biodiversity Strategy and Action Plan 2014 and Forest Policy 2015 also emphasize sustainable use and management of NTFPs and critically provide special opportunity to support livelihoods of marginalized propoor and women through wise use of NTFP. Nevertheless, present policy formation, implementation and field reality reflects power structures and domination by certain stakeholders and interests [60].

Gender participation in policy formulation is also challenging. For example, $75 \%$ of people collecting
NTFPs in India were women and $100 \%$ involved in NTFP processing were women, but their inclusion in Joint Forest Management committees was less than $10 \%$ [61]. Similarly, in Nepal, although women contribute a large share of the labor for forest and biodiversity conservation in community forests, they represent only $22 \%$ in the executive bodies of Community Forest User Groups [62]. Similar situation exists in Bhutan where the women's involvement is generally low in the designing, planning, and implementation of forestry policies, and there is limited understanding of the roles, knowledge, aspirations and contributions made by women towards NTFP management [63].

Considerable efforts have been made to develop the NTFP sector, but the contribution of NTFPs in national economies remains insignificant. As pointed by Shackleton and Pandey [21], the reason behind this is that their economic value remains invisible to external observers as most NTFPs are used for household purposes; production and harvest of NTFPs is a seasonal event, with their use or trade involving only small quantities; much of the NTFP trade is via informal and closed markets which are hard to enumerate; production and markets is dispersed; and their use is highest in rural areas, which are often remote and marginalised in terms of human resources and development policies.

\section{Gaps on knowledge based conservation and management of NTFPs}

Like in other parts of the Himalayas, there is still a severe paucity of in-depth field based information on the abundance, reproductive biology and ecological impacts of harvesting of NTFPs in the Kangchenjunga Landscape. There is no standard method available to estimate the economic contribution of NTFPs and their products. Research on the inventory, life history of NTFPs, and

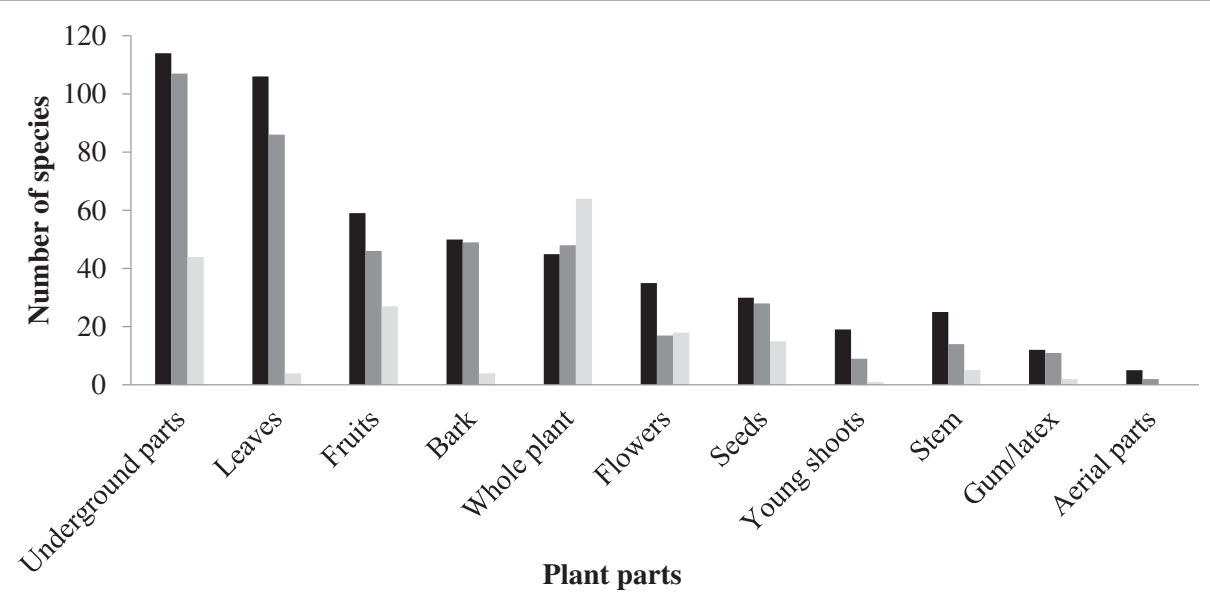

Fig. 5 Use frequency (number of species) of different plant parts in traditional medicine preparation in India (black bars), Nepal (dark grey bars), and Bhutan (light grey bars) 
Table 4 Major NTFPs traded (in kg) and revenue generated (USD) from 2008 to 2013 in the Nepal part of Kangchenjunga Landscape

\begin{tabular}{|c|c|c|}
\hline Species/products and parts & $\begin{array}{l}\text { Traded } \\
\text { quantity (kg) }\end{array}$ & $\begin{array}{l}\text { Revenue } \\
\text { (USD) }\end{array}$ \\
\hline Argeli (Edgeworthia gardneri)/Bark & 97,000 & 4109 \\
\hline Ban lasun (Fritillaria cirrhosa)/Bulb & 1500 & 150 \\
\hline Bish jara (Aconitum ferox)/Root & 4300 & 301 \\
\hline Chiraito (Swertia chirayita)/Whole plant & 88,765 & 7445.97 \\
\hline Chutro (Berberis wallichiana)/Bark & 5000 & NA \\
\hline Daruhaldi (Mahonia napaulensis)/Bark & 6500 & 130 \\
\hline Dhupi pat (Juniperus indica)/Twig & 3800 & 76 \\
\hline Khayar (Acacia catechu)/Heartwood & $97,784.6$ & $38,456.88$ \\
\hline Lauth salla (Taxus wallichiana)/Twig & 290,500 & 9441.25 \\
\hline Lichen (Usnea sp., Parmelia sp.)/Whole plant & 11,000 & 1650 \\
\hline Lokta (Daphne bholua)/Bark & 71,076 & 1940 \\
\hline Majitho (Rubia manjith)/Whole plant & 78,800 & 2199.24 \\
\hline $\begin{array}{l}\text { Nagbeliko powder (Lycopodium } \\
\text { clavatum)/Pollen grains }\end{array}$ & 8000 & 160 \\
\hline Khoto (Pinus roxburghii)/Resin & $1,256,334$ & 9799.40 \\
\hline Ritha (Sapindus mukorossi)/Fruit & 1600 & 48 \\
\hline Total & $2,021,959.6$ & $75,906.74$ \\
\hline
\end{tabular}

Source: Compiled from Hamro Ban - a yearly publication of Department of Forests, Ministry of Forests and Soil Conservation, Government of Nepal

impact of harvesting on the ecosystem is a prerequiste for their sustainable management, yet very few such activities have been documented from the landscape [64]. Similarly, ecological impacts of NTFPs harvesting for domestic and commercial purposes must be estimated to ensure their sustainability and the implementation for effective conservation measures.
Market size, structure and value chain of NTFP species depend on the demand and supply characteristics of products and their beneficiaries in different areas. Understanding market information is important for value addition and in devising investment strategies [16] for NTFPs based products, their diversification, and related enterprises. At present, the majority of NTFPs from the Kangchenjunga Landscape are traded in the raw forms, and NTFP harvesters lack necessary support for market-based strategies from both private, as well as government sectors.

Indigenous knowledge and management systems have been recognized for contributing to sustainable use of NTFPs, and consequently they have secured legal rights to manage forest resources [55]. In the Kangchenjunga Landscape, indigenous knowledge on NTFP use is well documented, but indigenous management systems need to be assessed and used for sustainable management.

\section{Conclusion}

We documented NTFPs collected and used for various purposes by the local people of the Kangchenjunga Landscape. The diversity of NTFPs was highest in India, followed by Nepal and Bhutan. Though the landscape possesses many potential species for trade, their nominal contribution to local livelihoods was due to lack of value addition and commercialization. Unsustainable harvesting and lack of value addition and commercialization could be considered as major challenges for conservation and development of the NTFP sector in the landscape.

Tracing the trend of NTFP research and exploitation, it shows much focus on medicinal plants resulting in overharvesting of some highly potential medicinal plants, with

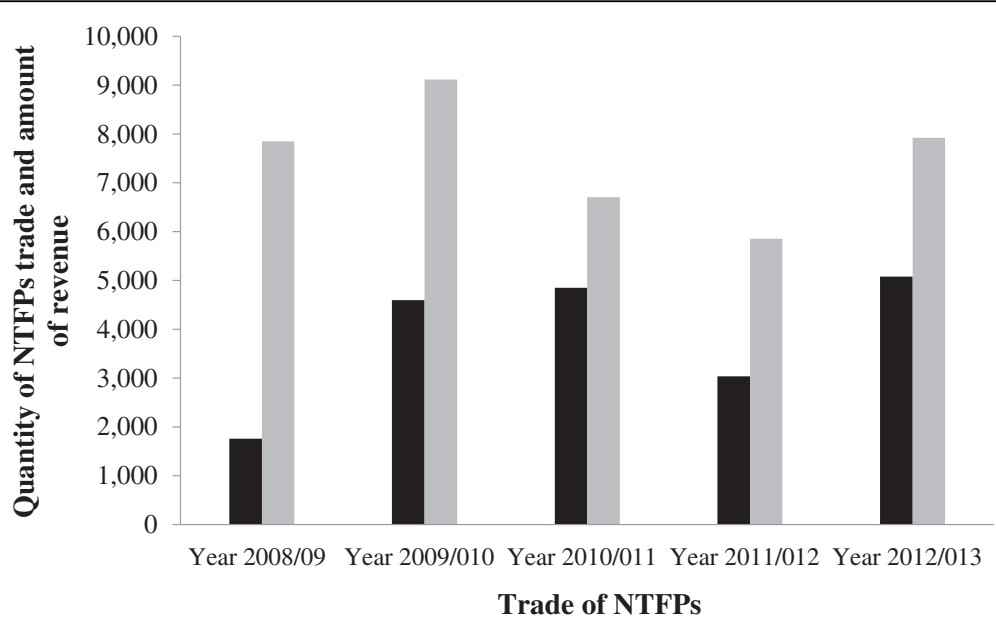

Fig. 6 Amount of traded NTFPs (black bars) in '000 kg and revenue generated (grey bars) in USD in five years in the Nepalese part of the Kangchenjunga Landscape 
very negligible amount of other plant species reported for other purposes. At present, NTFPs are synonymous with medicinal and aromatic plants and vice versa. Only small amounts of other NTFPs are marketed. Therefore, research must also focus on other potential categories of NTFPs. NTFP collectors need to be educated about forest ecology and the adverse impacts of unsustainable harvesting for conservation and local livelihoods. Sustainable harvesting techniques should be provided through training and capacity building programs to local people. Biological studies of high value NTFPs must be carried out in order to ensure sustainability of these resources.

Phytochemical screening of medicinal plants and nutrient value analysis of wild edible plants would foster their commercialization. Traditional knowledge of medicinal plant use could be integrated with 'modern' health care systems [65]. Highly potential NTFPs must be identified and grown for commercial cultivation and adopted in traditional agroforestry systems. This will reduce pressure on these species in their natural environments while providing economic benefits to poor farmers [47].

Conservation and development organizations, together with government agencies and private sectors, must provide technical and innovative inputs to add value to NTFP products. They must also facilitate community mobilization for assessment and identification of potential NTFPs. The latter role will be of significant importance considering the limited human and financial resources of government agencies in the Kangchenjunga Landscape. An integrated approach will promote sustainable use of NTFPs while contributing to income generation and livelihood improvement for local people. Transboundary landscape conservation programs will provide opportunities for transboundary cooperation through policy reforms, as well as providing opportunities to diversify livelihoods of forest dependent communities. However, marketing and commercialization of NTFPs can be successful only if the activity is transparent, equitable and sustainable, with important implications for poverty reduction and better resource management [20]. Increasing access to NTFP-selling outlets could be achieved through information dissemination, empowerment of collectors and establishment of linkages between collectors and traders [41]. The role of small and medium sized enterprises and cooperatives is extremely important to achieve sustainable management of NTFPs.

\section{Competing interests}

The authors declare that they have no competing interests.

\section{Authors' contributions}

YU, RCP, NC, and RPC designed the study. YU, RCP and NC carried out the literature search. YU, RCP, JG, NC and RPC analyzed the data and wrote the manuscript. All authors approved the final version of the manuscript.

\section{Acknowledgements}

The authors would like to recognize the support of the Austrian Development Agency (ADA) and the German Agency for International Cooperation (GIZ) for their financial support through International Centre for Integrated Mountain Development (ICIMOD).

\section{Author details}

${ }^{1}$ Research Centre for Applied Science and Technology, Tribhuvan University, Kirtipur, Kathmandu, Nepal. ${ }^{2} \mathrm{Nepal}$ Academy of Science and Technology (NAST), Khumaltar, Lalitpur, Nepal. ${ }^{3}$ International Centre for Integrated Mountain Development, Khumaltar, Lalitpur, Nepal. ${ }^{4}$ Central Department of Botany, Tribhuvan University, Kirtipur, Kathmandu, Nepal.

Received: 21 October 2015 Accepted: 3 April 2016

Published online: 03 May 2016

\section{References}

1. MEA (Millennium Ecosystem Assessment). Ecosystems and Human Well-Being: Synthesis. Washington: Island Press; 2005.

2. Ticktin $T$. The ecological implications of harvesting non-timber forest products. J Appl Ecol. 2004;41(1):11-21.

3. Belcher B, Ruíz-Pérez M, Achdiawan R. Global patterns and trends in the use and management of commercial NTFPs: implications for livelihoods and conservation. World Dev. 2005;33(9):1435-52.

4. Rasul G, Karki M, Sah RP. The role of non-timber forest products in poverty reduction in India: Prospects and problems. Develop Pract. 2008;18(6):779-88

5. Steele MZ, Shackleton CM, Shaanker RU, Ganeshaiah KN, Radloff S. The influence of livelihood dependency, local ecological knowledge and market proximity on the ecological impacts of harvesting non-timber forest products. Forest Policy Econ. 2015;50:285-91.

6. Shackleton C, Shackleton S. The importance of non-timber forest products in rural livelihood security and as safety nets: a review of evidence from South Africa. S Afr J Sci. 2004;100(11 \& 12):658-64.

7. Vedeld P, Angelsen A, Bojö J, Sjaastad E, Kobugabe Berg G. Forest environmental incomes and the rural poor. Forest Policy Econ. 2007;9(7): 869-79.

8. Belcher B, Schreckenberg K. Commercialisation of non-timber forest products: A reality check. Develop Policy Rev. 2007;25(3):355-77.

9. FAO (Food and Agriculture Organization of the United Nations). Non-wood forest products for rural income and sustainable forestry. Non-Wood Forest Products, 7. Rome: FAO; 1995.

10. Chettri N, Sharma E, Lama SD. Non-timber forest produces utilization, distribution and status in a trekking corridor of Sikkim, India. Lyonia. 2005;81(1):93-108.

11. Pradhan BK, Badola HK. Ethnomedicinal plants used by Lepcha tribe of Dzong valley bordering Khangchendzonga Biosphere Reserve in north Sikkim, India. J Ethnobiol Ethnomed. 2008:4:22.

12. Uprety Y, Poudel RC, Shrestha KK, Rajbhandary S, Tiwari NN, Shrestha UB, Asselin $\mathrm{H}$. Diversity of use and local knowledge of wild edible plant resources in Nepal. J Ethnobiol Ethnomed. 2012;8:16.

13. Chaudhary RP. In: Devi S, editor. Biodiversity in Nepal: Status and conservation. Bangkok: Saharanpur (U.P.) India and Teckpress Books; 1998.

14. Uprety Y, Boon EK, Poudel RC, Shrestha KK, Rajbhandary S, Ahenken A, Tiwari NN. Non-timber forest products in Bardiya district of Nepal: Indigenous use, trade and conservation. J Hum Ecol. 2010;30(3):143-58.

15. Chaudhary RP. Forest conservation and environmental management in Nepal: a review. Biodivers Conserv. 2000;9:1235-60.

16. Heinen JT, Shrestha-Acharya R. The non-timber forest products sector in Nepal: Emerging policy issues in plant conservation and utilization for sustainable development. J Sustain For. 2011;30(6):543-63.

17. Uprety Y, Poudel RC, Asselin H, Boon EK, Shrestha KK. Stakeholder perspectives on use, trade, and conservation of medicinal plants in the Rasuwa District of Central Nepal. J Mountain Sci. 2011;8(1):75-86.

18. Larsen $\mathrm{HO}$, Olsen CS. Unsustainable collection and unfair trade? Uncovering and assessing assumptions regarding central Himalayan medicinal plant conservation. Biodivers Conserv. 2007;16:1679-97.

19. Marshall E, Newton AC, Schreckenberg K. Commercialisation of non-timber forest products: first steps in analysing the factors influencing success. Int For Rev. 2003;5(2):128-37. 
20. Ghate R, Mehra D, Nagendra H. Local institutions as mediators of the impact of markets on non-timber forest product extraction in central India. Environ Conser. 2009;36:51-61.

21. Shackleton CM, Pandey AK. Positioning non-timber forest products on the development agenda. Forest Policy Econ. 2014;38:1-7.

22. ICIMOD, WCD, GBPIHED, RECAST, MoFSC. Feasibility Assessment Report. Regional Synthesis (draft). Kathmandu: ICIMOD; 2015.

23. Mittermeier RA, Gils PR, Hoffman M, Pilgrim J, Brooks T, Mittermeier CG, Lamoreaux J, daFonseca GAB (eds.). Hotspots Revisited. Earth's Biologically Richest and Most Endangered Terrestrial Ecoregions. Mexico City: CEMEX/ Agrupación Sierra Madre; 2004.

24. Pant KP, Rasul G, Chettri N, Rai KR, Sharma E. Value of Forest Ecosystem Services: A Quantitative Estimation from Eastern Nepal, Kangchenjunga Landscape. Kathmandu: ICIMOD; 2012.

25. Phuntsho K, Chettri N, Oli KP. Mainstreaming community-based conservation in a transboundary mountain landscape -Lessons from Kangchenjunga. Kathmandu: ICIMOD; 2012.

26. Chettri N, Sharma E, Shakya B, Thapa R, Bajracharya B, Uddin K, Oli KP, Choudhury D. Biodiversity in the Eastern Himalayas: Status, trends and vulnerability to climate change. Climate change impact and vulnerability in the Eastern Himalayas - Technical report 2. Kathmandu: ICIMOD; 2012

27. Chettri N, Sharma E, Shakya B, Bajracharya B. Biodiversity conservation beyond boundaries: An initiative on regional cooperation in the Hindu Kush-Himalaya. In: Bajracharya SB, Dahal N, editors. Shifting Paradigms in Protected Areas Management. Kathmandu: National Trust for Nature Conservation; 2008.

28. Cook FEM. Economic Botany Data Collection Standard. Kew: Royal Botanic Gardens; 1995

29. Kandel P, Gurung J, Chettri N, Ning W, Sharma E. Biodiversity research trends and gap analysis from a transboundary landscape, Eastern Himalayas. J Asia-Pacific Biodivers. 2016:9:1-10.

30. Coe FG, Anderson GJ. Ethnobotany of the Garifuna of Eastern Nicaragua. Econ Bot. 1996:50:71-107.

31. Collins S, Martins X, Mitchell A, Teshome A, Arnason JT. Quantitative ethnobotany of two East Timorese cultures. Econ Bot. 2006;60(4):347-61.

32. Koirala M. Non-timber forest products as alternative livelihood options in the Transborder villages of Eastern Nepal. In: Chettri N, Shakya B, Sharma E, editors. Biodiversity conservation in the Kangchenjunga landscape. Kathmandu: ICIMOD; 2008. p. 105-10.

33. Lama YC, Ghimire SK, Aumeeruddy-Thomas Y. Medicinal plants of Dolpo: Amchis' Knowledge and Conservation. Kathmandu: WWF Nepal Program; 2001.

34. Heinrich M, Rimpler H, Barrera NA. Indigenous phytotherapy of gastro- intestinal disorders in a lowland Mixe community (Oaxaca, Mexico): ethno-pharmacologic evaluation. J Ethnopharmacol. 1992;36:63-80.

35. Rokaya MB, Uprety Y, Poudel RC, Timsina B, Münzbergová Z, Asselin H, Tiwari A, Shrestha SS, Sigdel SR. Traditional uses of medicinal plants in gastrointestinal disorders in Nepal. J Ethnopharmacol. 2014;158:221-9.

36. Moore PD. Trials in bad taste. Nature. 1994;370:410-1.

37. Samant SS, Butola JS, Sharma A. Assessment of Diversity, Distribution, Conservation Status and Preparation of Management Plan for Medicinal Plants in the Catchment Area of Parbati Hydroelectric Project Stage - III in Northwestern Himalaya. J Mountain Sci. 2007:4(1):34-56.

38. Uprety Y, Asselin H, Boon EK, Yadav S, Shrestha KK. Indigenous use and bio-efficacy of medicinal plants in Rasuwa district, Central Nepal. J Ethnobiol Ethnomed. 2010;6:3.

39. Edwards DM. The Marketing of Non-Timber Forest Products from the Himalayas: The trade between East Nepal and India. Rural Development Forestry Network Paper No. 15b. London: Overseas Development Institute; 1993.

40. Edwards DM. The trade in non-timber forest products from Nepal. Mountain Res Develop. 1996;383-394.

41. Bista S, Webb EL. Collection and marketing of non-timber forest products in the far western hills of Nepal. Environ Conserv. 2006;33(03):244-55.

42. Delang CO. Not just minor forest products: The economic rationale for the consumption of wild food plants by subsistence farmers. Ecol Econ. 2006; 59(1):64-73

43. Chaudhary RP, Uprety $Y$, Joshi SP, Shrestha KK, Basnet K, Basnet G, Shrestha KR, Bhatta, KP, Acharya KP, Chettri N. Kangchenjunga Landscape Nepal: from conservation and development perspectives. Kathmandu: Ministry of Forests and Soil Conservation, Research Centre for Applied Science and Technology and International Centre for Integrated Mountain Development; 2015.
44. Carvalho AR. Popular use, chemical composition and trade of Cerrado's medicinal plants (Goias, Brazil). Environ Develop Sustain. 2004;6:307-16.

45. Hamilton A. Medicinal plants, conservation and livelihoods. Biodivers Conserv. 2004;13:1477-517.

46. Pyakurel D, Oli BR. Market study of tradable and economically important medicinal and aromatic plants of eastern Nepal. Thapathali: A report submitted to Department of Plant Resources; 2013.

47. Sundriyal M, Sundriyal RC. Underutilized edible plants of the Sikkim Himalaya: Need for domestication. Curr Sci. 2003;85(6):731-6.

48. Choudhary D, Kala SP, Todaria NT, Dasgupta S, Kollmair M. Drivers of Exploitation and Inequity in Non-Timber Forest Products (NTFP) Value Chains: The Case of Indian Bay Leaf in Nepal and India. Develop Policy Rev. 2014;32:71-87.

49. Maity D, Pradhan N, Chauhan AS. Folk uses of some medicinal plants from North Sikkim. Indian J Traditional Knowledge. 2004;3(1):72-9.

50. NCDC (Namsaling Community Development Centre): Environmental services provided by the biological corridor in Kanchenjunga Singhalila Complex Eastern Nepal. A report submitted to ICIMOD, Kathmandu. Ilam: NCDC; 2010.

51. Paudel GP. Comprehensive socio-economic baseline survey of selected SCAPES VDCs of Taplejung and Panchthar districts in Nepal. Kathmandu: WWF-Nepal; 2010.

52. Bantawa P, Rai R. Studies on ethnomedicinal plants used by traditional practitioners, Jhankri, Bijuwa and Phedangma in Darjeeling Himalaya. Natural Product Radiance. 2009;8(5):537-41.

53. Limbu DK, Rai BK. Ethno-medicinal practices among the Limbu community in Limbuwan, Eastern Nepal. Glob J Hum Soc Sci. 2013;2:7-29.

54. Shanley P, Pierce AR, Laird SA, Guillen SA. Tapping the Green Market: Management and Certification of Non-timber Forest Products. London: Earthscan Publications; 2002.

55. Ghimire SK, Sapkota IB, Oli BR, Parajuli-Rai R. Non-Timber Forest Products of Nepal Himalayas: Database of Some Important Species Found in the Mountain Protected Areas and Surronding Regions. Kathmandu: WWF Nepal; 2008

56. DPR (Department of Plant Resources). Plant resources (a newsletter) Government of Nepal, Ministry of Forests and Soil Conservation. Thapathali: Department of Plant Resources; 2004.

57. Ugyen PW, Olsen A. Vulnerable medicinal plants and the risk factors for their sustainable use in Bhutan. J Bhutan Stud. 2008:19:66-90.

58. MoEF (Ministry of Environment and Forests). National Forest Policy. New Delhi: Government of India; 1988.

59. MoFSC (Ministry of Forests and Soil Conservation). Herbs and Non-Timber Forest Products Development Policy 2004. Kathmandu: Ministry of Forests and Soil Conservation, Herbs and NTFPs Coordination Committee; 2004.

60. Larsen HO, Olsen CS, Boon TE. The non-timber forest policy process in Nepal: actors, objectives and power. Forest Policy Econ. 2000;1(3):267-81.

61. Sarker D, Das N. Women's participation in forestry: some theoretical issues. Econ Pol Wkly. 2002:37(43):4407-12.

62. DoF (Department of Forest). Community Forestry Database. Department of Forest, Ministry of Forests and Soil Conservation. Kathmandu: Government of Nepal; 2012.

63. SFD (Social Forestry Division): National strategy for the development of Non-Wood Forest Products in Bhutan, 2008-2018. Social Forestry Division, Ministry of Agriculture, Royal Government of Bhutan, 2008.

64. Canon PF, Hywel-Jones NL, Maczey N, Norbu L, Samdup T, Lhendup P. Steps towards sustainable harvest of Ophiocordyceps sinensis in Bhutan. Biodivers Conserv. 2009;18(9):2263-81.

65. Falconer J. The major significance of 'minor' forest products: the local use and value of forests in the west African humid forest zone. Rome: FAO; 1990.

66. Siwakoti M, Siwakoti S. Ethnomedicinal uses of plants among the Satar tribe of Nepal. J Econ Taxonomic Bot. 2000;24:323-33.

67. Gautam TP. Indigenous uses of some medicinal plants in Panchthar district, Nepal. Nepalese J Biosci. 2011;1:125-30

68. Rai SK. Medicinal plants used by Meche people of Jhapa district, eastern Nepal. Our Nature. 2004;2:27-32.

69. Hussain S, Hore DK. Collection and conservation of major medicinal plants of Darjeeling and Sikkim Himalayas. Indian J Traditional Knowledge. 2007:6(2):352-7.

70. Tshering S. Natural vegetable dyes; food, fruit species and mushrooms; gums and waxes; and incense. In: Non-wood forest products of Bhutan. Bangkok: FAO; 1996. p. 87-106. 
71. Nawang R. Medicinal plants. In: Non-wood forest products of Bhutan. Bangkok: FAO; 1996. p. 13-20.

72. Avasthe RK, Rai PC, Rai LK. Sacred groves as repositories of genetic diversity-a case study from kabi-longchuk. North Sikkim ENVIS Bulletin: Himalayan Ecology. 2004;12(1):25.

73. Parajuli RR. Indigenous Knowledge on Medicinal Plants: Maipokhari, Maimajhuwa and Mabu VDCs of Ilam District, Eastern Nepal. J Dept Plant Resour Nepal. 2013;35:50-8.

74. Saha J, Sarkar PK, Chattopadhyay S. A survey of ethnomedicinal plants of Darjeeling hills for their antimicrobial and antioxidant activities. Indian J Nat Prod Resour. 2011;2(4):479-92.

75. Lepcha SR, Das AP. Ethno-medicobotanical exploration along the international borders to Tibet Autonomous Region of China and the kingdom of Bhutan with special reference to the Pangolakha Wildlife Sanctuary. East Sikkim: Recent Studies in Biodiversity and Traditional Knowledge in India; 2011. p. 257-70.

76. Parajuli RR. Ethnomedicinal Use of Plants in Rai community of Maimajuwa and Puwamajuwa VDCs of Ilam District, Eastern Nepal. Bull Dept Plant Res. 2012;34:65-73.

77. Parajuli RR. Study on Ethnobotanical Plants of Maipokhari Wetland Area in llam, Eastern Nepal. Bull Dept Plant Res. 2011;33:33-42.

78. Chhetri DR. Medicinal plants used as antipyretic agents by the traditional healers of Darjeeling Himalayas. Indian J Traditional Knowledge. 2004;3(3):271-5.

79. Badola HK, Pradhan BK. Plants used in healthcare practices by Limboo tribe in South-West of Khangchendzonga Biosphere Reserve, Sikkim. Indian J Traditional Knowledge. 2013;12(3):355-69.

80. Chettri N. Impact of habitat disturbances on bird and butterfly communities along the Yuksam-Dzongri trail in Khangchendzonga Biosphere Reserve. Ph. D. thesis. India: University of North Bengal; 2000.

81. Sherpa S. The high altitude ethnobotany of the Walung people of Walangchung Gola, Kanchanjunja Conservation Area, east Nepal, Master thesis. Nepal: Central Department of Botany, Tribhuvan University, Kathmandu; 2001.

82. Shrestha KK, Ghimire SK. Diversity, ethnobotany and conservation strategy of some potential medicinal and aromatic plants of Taplejung, east Nepal (Tamur valley). Kathmandu: Report submitted to ANSAB; 1996.

83. WWF-Nepal: Gift of the Himalayas: Non timber forest products of the Sacred Himalayan Landscape-Nepal. WWF Nepal, 2007.

84. Bharati KV, Sharma BL. Some ethnoveterinary plant records for Sikkim Himalaya. Indian J Traditional Knowledge. 2010;9(2):344-6.

85. Chanda R, Mohanty JP, Bhuyan NR, Kar PK, Nath LK. Medicinal plants used against gastrointestinal tract disorders by the traditional healers of Sikkim Himalayas. Indian J Traditional Knowledge. 2007:6(4):606-10.

86. Moktan S, Das AP. Ethnomedicinal approach for diarrhoeal treatment in Darjiling district (WB), India. Ethnobotany. 2013;25:160-3.

87. Ghimire SK, Nepal BK. Developing a Community-based Monitoring System and Sustainable Harvesting Guidelines for Non-Timber Forest Products (NTFPs) in Kangchenjunga Conservation Area (KCA). East Nepal. Unpublished report. Kathmandu: WWF Nepal; 2008.

88. Oli BR, Nepal BK. Non-timber forest products from the Kangchenjunga Conservation Area: Aspects of trade and market opportunities. Kathmandu: WWF Nepal Programme; 2003.

89. Chhetri DR, Basnet D, Chiu PF, Kalikotay S, Chhetri G, Parajuli S. Current status of ethnomedicinal plants in the Darjeeling Himalaya. Curr Sci. 2005;89(2):264-8.

90. Singh HB, Sundriyal RC. Composition, economic use, and nutrient contents of Alpine vegetation in the Khangchendzonga Biosphere Reserve, Sikkim Himalaya, India. Arctic Antarctic Alpine Res. 2005;37(4):591-601.

91. Pal S, Palit D. Traditional knowledge and bioresource utilization among Lepcha in North Sikkim. NeBIO. 2011;2(1):13-7.

92. Siwakoti M, Siwakoti S, Varma SK. Ethnobotanical notes on wild edible plants used by Satars of Nepal. Tribhuvan University J. 1997;20(1):57-64.

93. Chamling KD. Traditional paper, essential oils, rosin and turpentine. In: Non-wood forest products of Bhutan. Bangkok: FAO; 1996. p. 43-60.

94. Ghimeray AK, Sharma P, Ghimire B, Lamsal K, Ghimire B, Cho DH. Wild edible flowering plants of the Illam Hills (Eastern Nepal) and their mode of use by the local community. Korean J Plant Taxonomy. 2010;40(1):1-4.

95. Sundriyal M, Sundriyal RC. Wild edible plants of the Sikkim Himalaya: Nutritive values of selected species. Econ Bot. 2001;55(3):377-90.

96. Pradhan S. Antihyperglycemic effect of various medicinal plants of Sikkim Himalayas-A review. Int J Res Phytochem Pharmacol. 2011;1(3):124-30.
97. Bharati KA, Sharma BL. Plants used as ethnoveterinary medicines in Sikkim Himalayas. Ethnobotany Res Appl. 2010;10:339-56.

98. Lachungpa U. Indigenous lifestyles and biodiversity conservation issues in North Sikkim. Indian J Traditional Knowledge. 2009;8(1):51-5.

99. Wangyal JT. Ethnobotanical knowledge of local communities of Bumdeling wildlife sanctuary, Trashiyangtse, Bhutan. Indian J Traditional Knowledge. 2012;11:447-52.

100. Rinchen D. Bamboo, cane, wild banana, fibre, floss and brooms. In: Non-wood forest products of Bhutan. Bangkok: FAO; 1996. p. 13-20.

101. Tamang B, Tamang JP. Traditional knowledge of biopreservation of perishable vegetable and bamboo shoots in Northeast India as food resources. Indian J Traditional Knowledge. 2009;8(1):89-95.

102. Matsushima K, Minami M, Nemoto K. Use and conservation of wild plants of Bhutan. J Faculty Agricult Shinshu University. 2012;48(1-2):75-83.

103. Phoboo S, Jha PK. Trade and Sustainable Conservation of Swertia chirayita (Roxb. ex Fleming) h. Karst in Nepal. Nepal J Scie Technol. 2010;11:125-32.

104. Poudel RC, Gao LM, Möller M, Baral SR, Uprety Y, Liu J, Li DZ. Yews (Taxus) along the Hindu Kush-Himalayan region: exploring the ethnopharmacological relevance among communities of Mongol and Caucasian origins. J Ethnopharmacol. 2013;147(1):190-203.

105. Sarkar A, Das AP. Ethonobotanical formulation for the treatment of jaundice by Mech tribe in Duars of West Bengal. Indian J Traditional Knowledge. 2010;9(1):34-136.

106. Sharma P, Uniyal PL. Traditional knowledge and conservation of Taxus baccata in Sikkim Himalaya. NeBIO. 2010;1(1):55-9.

107. Subba JR. Indigenous knowledge on bio-resources management for livelihood of the people of Sikkim. Indian J Traditional Knowledge. 2009;8(1):56-64.

108. Tamang JP, Sarkar PK, Hesseltine CW. Traditional fermented foods and beverages of Darjeeling and Sikkim-a review. J Sci Food Agric. 1988;44(4):375-85. 